\title{
FABRICATION AND CHARACTERIZATION OF ADHERENT DIAMOND-LIKE CARBON BASED THIN FILMS ON POLYETHYLENE TEREPHTHALATE BY END HALL ION BEAM DEPOSITION
}

\author{
A Thesis Submitted to the College of \\ Graduate Studies and Research \\ In Partial Fulfillment of the Requirements \\ For the Degree of Master of Science \\ In the Department of Mechanical Engineering \\ University of Saskatchewan \\ Saskatoon \\ Canada
}

By

Parisa Ashtijoo

(C) Copyright Parisa Ashtijoo, August 2016. All rights reserved. 


\section{PERMISSION TO USE}

In presenting this thesis in partial fulfillment of the requirements for a postgraduate degree from the University of Saskatchewan, I agree that the Libraries of this University may make it freely available for inspection. I further agree that permission for copying of this thesis in any manner, in whole or in part, for scholarly purposes may be granted by the professor who supervised my thesis work or, in their absence, by the Head of the Department or the Dean of the College in which my thesis work was done. It is understood that any copying or publication or use of this thesis or parts thereof for financial gain shall not be allowed without my written permission. It is also understood that due recognition shall be given to me and to the University of Saskatchewan in any scholarly use which may be made of any material in my thesis.

Requests for permission to copy or to make other use of material in this thesis in whole or part should be addressed to:

Head of the Department of Mechanical Engineering

57 Campus Dr.

University of Saskatchewan

Saskatoon, Saskatchewan (S7N 5A9) 


\begin{abstract}
The application of Polyethylene terephthalate (PET) has been steadily increasing during recent years. However, its low wear resistance, high gas permeation rate and poor biocompatibility have significantly limited its applications in some specific areas. On the contrary, diamond-like carbon (DLC) possesses many unique properties such as the high hardness, low friction coefficient, high transparency, good gas barrier, excellent biocompatibility, and low synthesis temperature, which makes it an ideal coating material for modifying the surfaces of PET for applications as food and beverage containers and biomedical implants. Nevertheless, the adhesion of DLC on PET is poor due to the high stress induced by ion bombardment during DLC deposition and the large mechanical property mismatch between the coating and the substrate. Therefore, it is very important to develop techniques to lower the stress of DLC and to enhance the adhesion strength of DLC to PET.

In the present thesis work, low energy end-hall (EH) ion source and element ( $\mathrm{N}$ and $\mathrm{Si}$ ) doping were used to deposit DLC to lower its internal stress and ion beam treatment was performed to modify the surface of PET to enhance the adhesion between DLC and PET. In addition, two different hydrocarbon gases $\left(\mathrm{CH}_{4}\right.$ and $\left.\mathrm{C}_{2} \mathrm{H}_{2}\right)$ were used as precursors for DLC deposition in order to understand the effect of precursor gas. The structure and properties of the treated PET and deposited DLC films were characterized by various advanced techniques: The adhesion of DLC to PET was assessed using scratch testing; the composition and bonding states of DLC and treated PET were analyzed by Raman spectroscopy, Fourier transform infrared spectroscopy (FTIR), X-ray photoelectron spectroscopy (XPS), and near edge X-ray absorption fine structure (NEXAFS); the hardness and Young's modulus were measured by nano-indentation; the tribological properties of the coated materials were evaluated by pin-on-disk sliding testing; and the surface tomography and surface roughness were investigated by atomic force microscopy (AFM), optical profiler, and water contact angle measurements. The main results are summarized as follows. Ion treatment can effectively change PET microstructure and chemical states and thus improve the adhesion of subsequent deposited DLC. The kind of ions and the treatment time play an important role in PET structure changes. Oxygen ion treatment can effectively change the chemical states of PET and improve the adhesion of DLC and can further improve DLC adhesion. Element doping can change the structure of DLC and thus the
\end{abstract}


mechanical properties. Si doping increases $\mathrm{sp}^{3}$ carbon bonding content in the films while $\mathrm{N}$ doping decreases $\mathrm{sp}^{3}$ carbon bonding content in the films. The doped $\mathrm{Si}$ forms $\mathrm{sp}^{3} \mathrm{Si}-\mathrm{C}$ bonds and $\mathrm{N}$ forms $\mathrm{sp}^{3} \mathrm{C}-\mathrm{N}$ and $\mathrm{sp}^{2} \mathrm{C}=\mathrm{N}$ bonds in the films. Consequently, the hardness values decrease from 11.5 GPa for pure DLC to 7.0 GPa for N-DLC samples whereas the hardness of Si-DLC increases with increasing the silicon content. In addition, $\mathrm{N}$ doping can reduce residual stress of DLC and thus increases adhesion of DLC. The DLC films deposited using methane show better properties than using acetylene. By optimizing the conditions, dense, smooth, and adhesive DLC films have been deposited onto PET using EH ion source by combining $\mathrm{N}$ doping and $\mathrm{O}$ ion treatment with methane as precursor gas. 


\begin{abstract}
AKNOWLEDGEMENTS
I would like to express my sincere gratitude to my supervisor, Professor Qiaoqin Yang for her continuous advice, support and encouragement throughout this research. Also, I would like to appreciate my committee member Professor Jerzy Szpunar and Professor Chris Zhang and Professor Lifeng Zhang for their valuable comments.

My special thanks go out to those who provided help in sample characterization: Professor Matthew Paige for AFM measurement, Dr. Ronny Sutarto for XPS measurement at CLS, Jason Maley for Raman spectroscopy at the Saskatchewan Structural Science Center (SSSC), Eric Neil for water contact angle measurements. I am also grateful to Robert Peace for his technical assistance and my colleague Santu Bhattacharya for his continuous help during this project.

The financial support granted by the Natural Sciences and Engineering Research Council of Canada (NSERC), the Canada Research Chair Program (CRC), and the University of Saskatchewan is greatly appreciated.

Finally, I would like to express my heartfelt gratitude to my family, specially my parents, for their continuous support and love during my life.
\end{abstract}




\section{DEDICATION}

TO MY BELOVED MOTHER AND FATHER 


\section{TABLE OF CONTENTS}

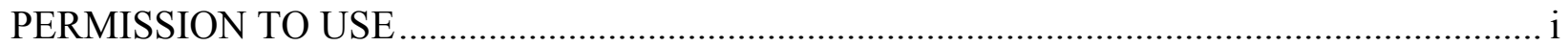

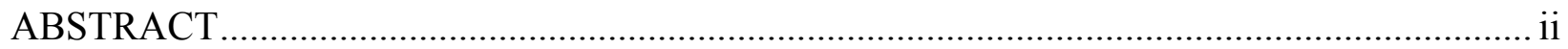

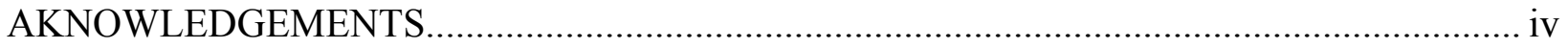

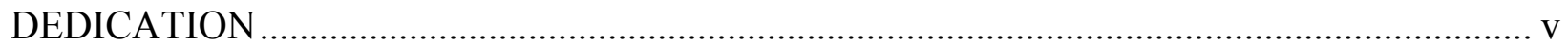

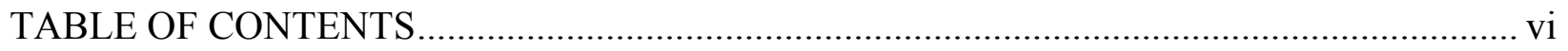

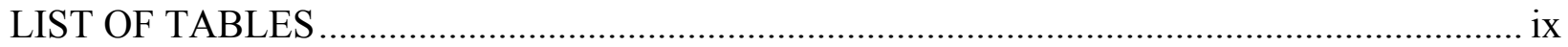

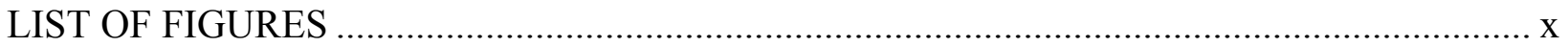

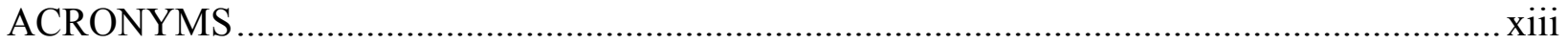

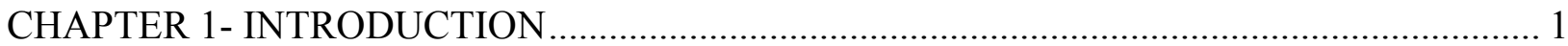

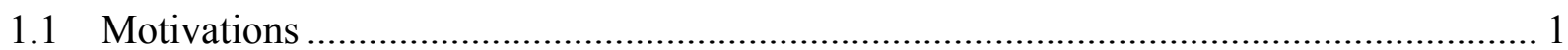

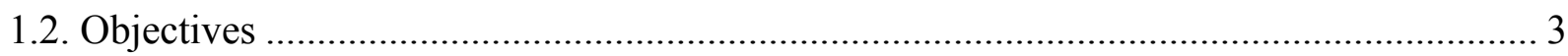

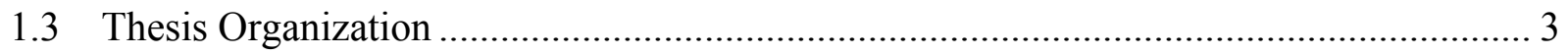

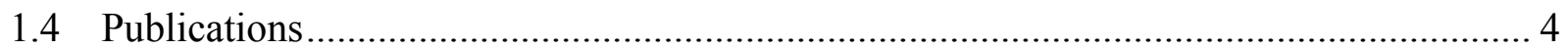

CHAPTER 2- LITERATURE REVIEW ..................................................................... 5

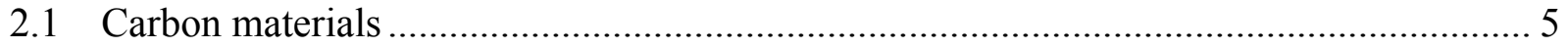

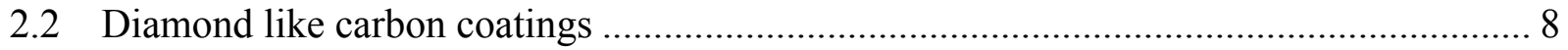

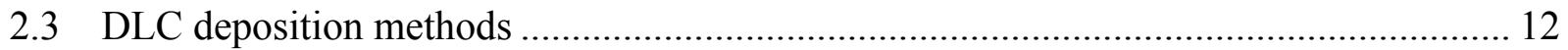

2.4 DLC deposition mechanism.......................................................................... 15

2.4.1 Growth of a-C:H films: ......................................................................... 16

2.5 Diamond-like carbon thin films on Polyethylene terephthalate (PET)....................... 17

2.6 Adhesion improvement of DLC on PET substrate ................................................... 19

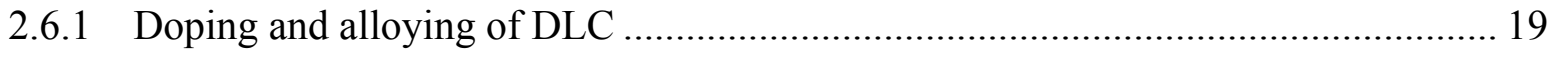

2.6.2 Polymer surface modification techniques .................................................. 23 
2.7 Structural characterization of diamond-like carbon thin films ...................................... 25

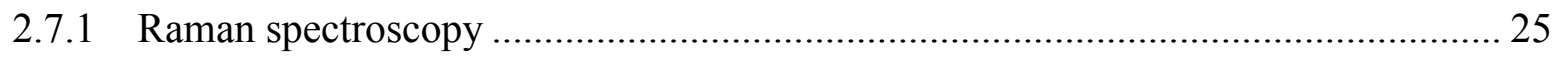

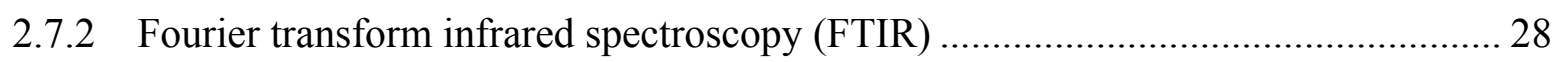

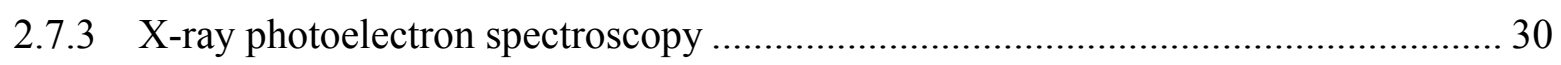

2.7.4 Near edge X-ray absorption fine structure spectroscopy ………………………..... 31

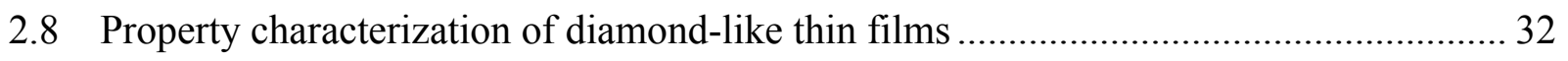

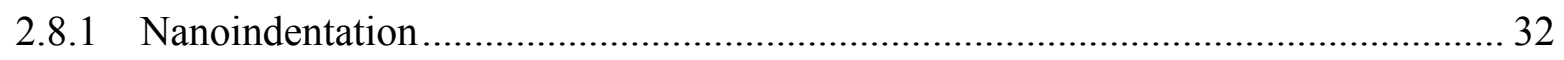

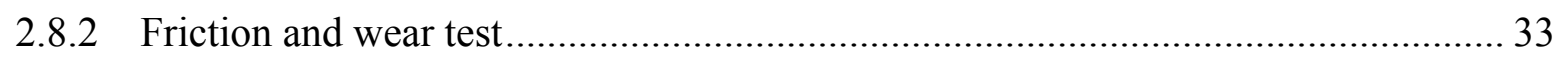

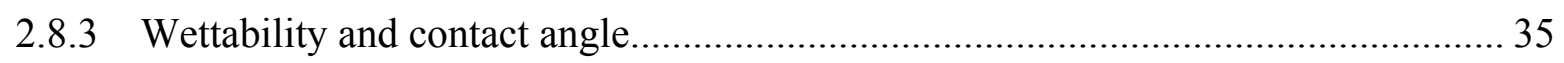

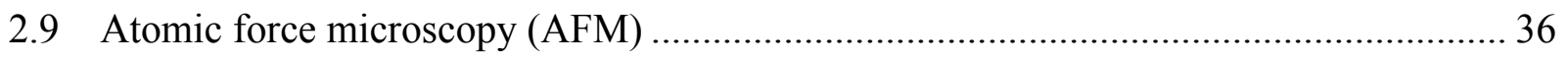

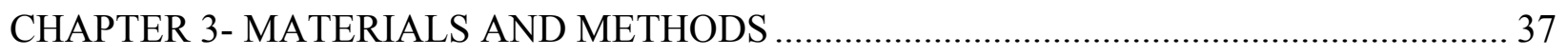

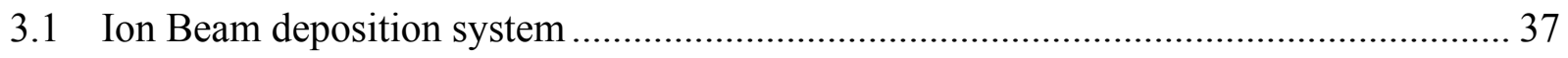

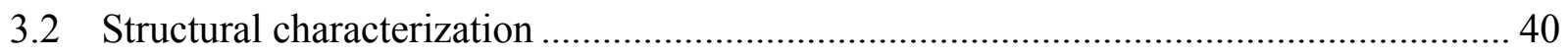

3.3 Surface property characterization ………………..................................................... 40

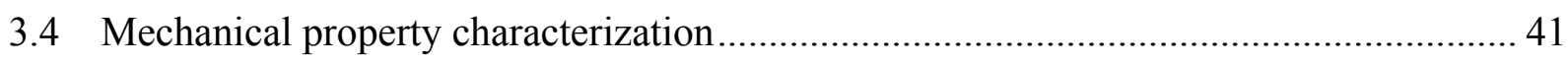

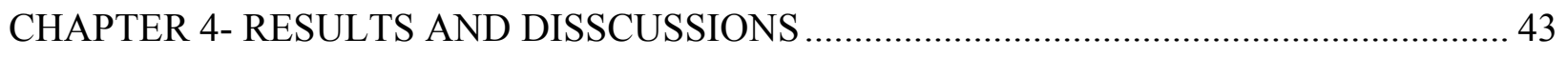

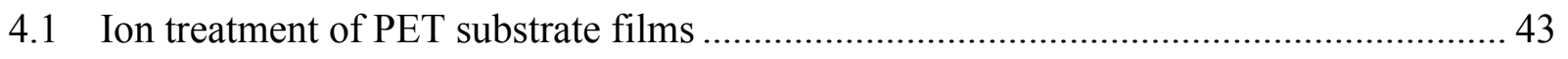

4.1.1 X-ray photoelectron spectroscopy (XPS) ……………………………………..... 43

4.1.2 Fourier transform infrared spectroscopy (FTIR) ………………………………. 47

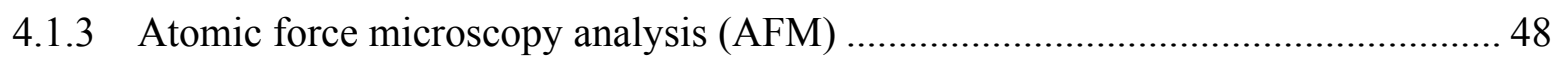

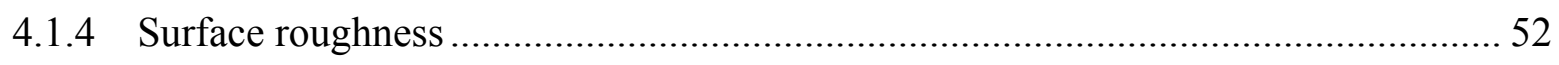

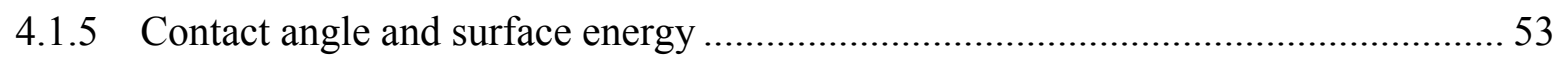

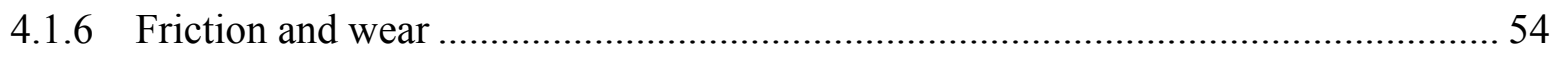

4.2 Effect of ion treatment of PET substrate films on DLC deposition................................. 56

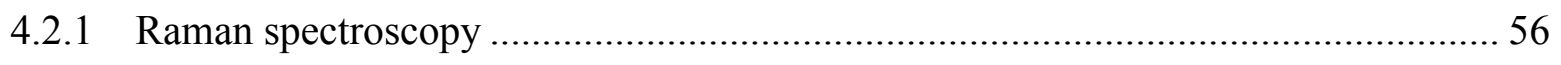




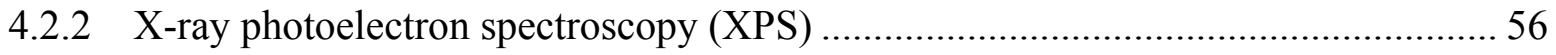

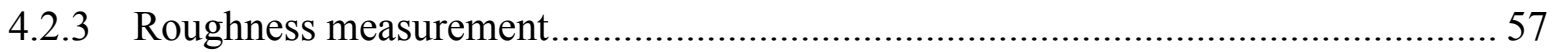

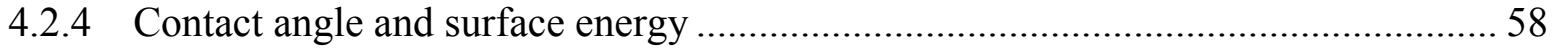

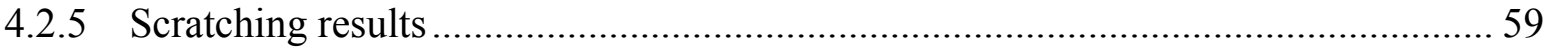

4.3 Effect of doping on the structure and properties of DLC on PET ………….................... 61

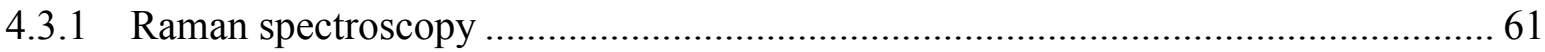

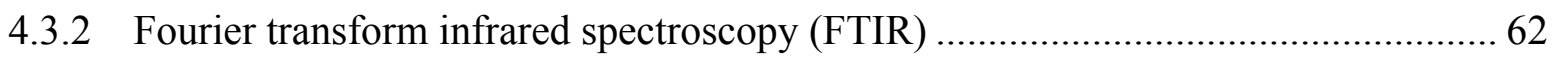

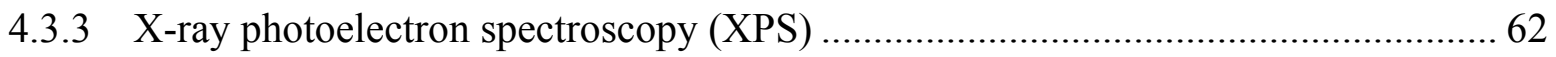

4.3.4 Near edge X-ray absorption fine structure (NEXAFS)............................................... 65

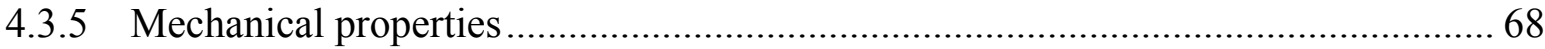

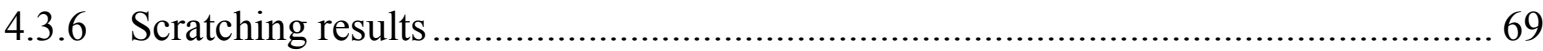

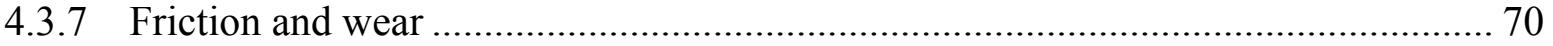

4.3.8 Contact angle and surface energy ……………….............................................. 71

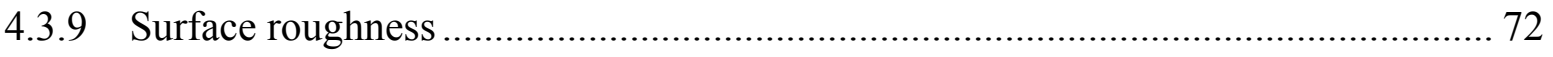

CHAPTER 5- SUMMARY, CONCLUSIONS, AND FUTURE WORK ……………………...... 74

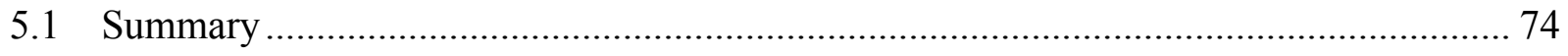

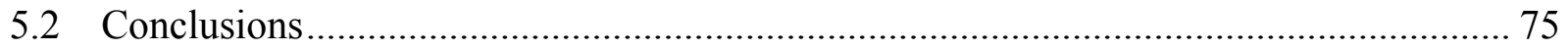

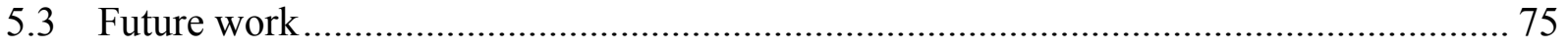

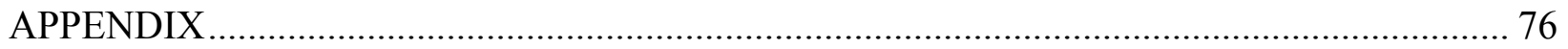

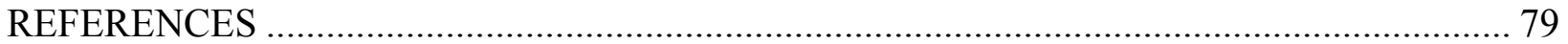




\section{LIST OF TABLES}

Table 2.1. List of DLC structure, composition and properties [30]............................................ 11

Table 3.1. Deposition parameters of doped DLC samples ......................................................... 38

Table 3.2. Deposition parameters of pure DLC with two different hydrocarbon gas ................... 39

Table 4.1. Bonding states of ion treated PET films ................................................................. 44

Table 4.2. O1s Bonding state of plasma treated PET films ......................................................... 46

Table 4.3. Roughness, water contact angle, and surface energy values of plasma treated PET... 53

Table 4.4. Roughness values of DLC-M and DLC-A deposited on ion treated films ................... 58

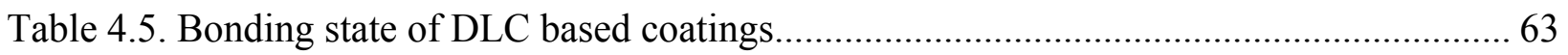

Table 4.6. Roughness, water contact angle and surface energy values for different DLC samples 


\section{LIST OF FIGURES}

Figure 2.1. Distribution of electrons in carbon orbitals [23] f............................................... 5

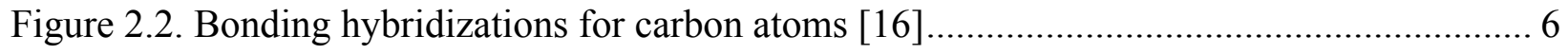

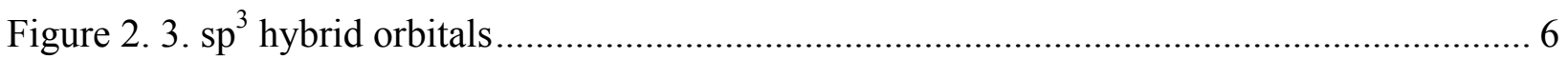

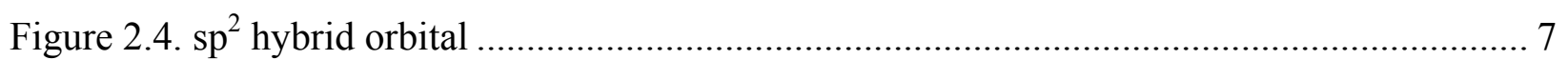

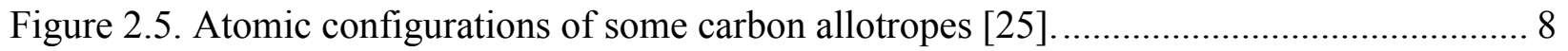

Figure 2.6. Ternary phase diagram of the C, H system [27] ............................................. 9

Figure 2.7. Atom number density vs. atom fraction of hydrogen [26] ..................................... 9

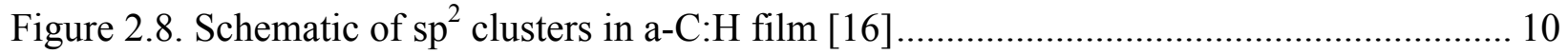

Figure 2.9. Molecular dynamic simulation of a-C:H film atomic structure [28]..................... 11

Figure 2.10. Schematic of different DLC deposition methods [16] ....................................... 13

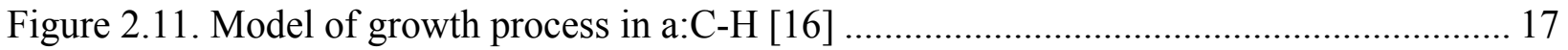

Figure 2.13. Polyethylene terephthalate (PET) structure ..................................................... 17

Figure 2.14. Schematic of most common doping elements in DLC for obtaining improved

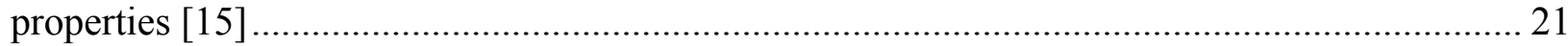

Figure 2.15. Carbon-Nitrogen bonding configurations [16] .............................................. 23

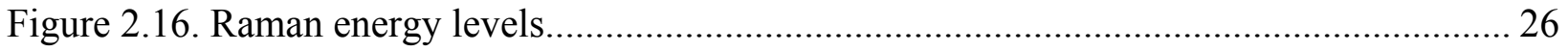

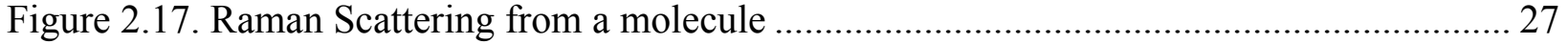

Figure 2.18. Raman Spectra of various carbon materials [16] …......................................... 27

Figure 2.19. Schematic of an amorphous carbon (a-C) D and G peaks. Arrows show the factors affecting the height and position of Raman D and G peaks of amorphous carbons. Dotted arrow indicates the indirect influence of the sp3 content on increasing $\mathrm{G}$ position [16] ..................... 29

Figure 2.20. The illustration of some of the molecule vibrations [79] ................................... 29

Figure 2.21. Schematic diagram of a) emission of photoelectron, b) auger, and c) fluorescence [23] 32

Figure 2.22. Loading -unloading curve from nano-indentation measurement [16] .................. 33

Figure 2.23. Schematic of three major components of friction [30] ...................................... 34

Figure 2.24. Schematic of ball-on-disc sliding test [84] ................................................... 35

Figure 2.25. Schematic of different contact angles............................................................ 36 


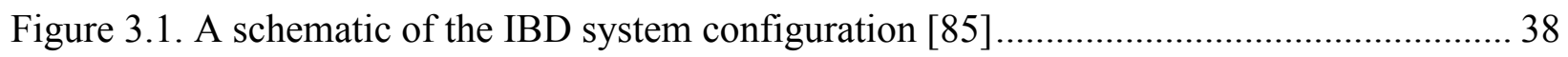

Figure 3.2. Schematic of image acquisition and contact angle measurement .............................. 42

Figure 4.1. De-convolution of XPS spectra of bare PET: a) C1s and b) O1s............................. 43

Figure 4.2. C1s XPS spectra of $\mathrm{Ar} / \mathrm{O}_{2}$ ion treated PET films a) for $\mathrm{r} 30 \mathrm{~min}$ and b) from $60 \mathrm{~min}$ 45

Figure 4.3. O1s XPS spectra of Oxygen treated PET film a) after $30 \mathrm{~min} \mathrm{~b}$ ) after $60 \mathrm{~min} . . . . . . . . .45$

Figure 4.4. C1s XPS spectra of Argon treated PET film a) for $30 \mathrm{~min}$ and b) for $60 \mathrm{~min} . . . . . . . . .46$

Figure 4.5. C1s XPS spectra of Argon treated PET film a) for $30 \mathrm{~min}$ and b) for $60 \mathrm{~min}$........... 47

Figure 4.6. ATR-FTIR Spectra of bare PET and 60 min Oxygen treated PET film .................... 47

Figure 4.7. AFM images of bare PET in a) $30 \mu \mathrm{m}$ and b) $1 \mu \mathrm{m}$ scales ...................................... 48

Figure 4.8. AFM images of PET films after oxygen ion treatment, a) 2D image after $30 \mathrm{~min}$ treatment, b) 2D image after 60 min treatment, c) 3D image after 30 min treatment, and d) 3D image after 60 min treatment

Figure 4.9. AFM images of PET surface after Nitrogen treatment, a) 2D image after $30 \mathrm{~min}$ treatment, b) 2D image after 60 min treatment, c) 3D image after 30 min treatment, and d) 3D

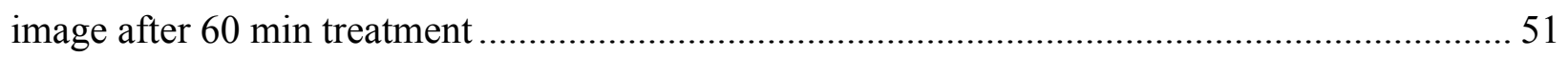

Figure 4.10. AFM images of PET surface after argon ion, a) 2D image after 30 min treatment, b) 2D image after 60 min treatment, c) 3D image after 30 min treatment, and d) 3D image after 60

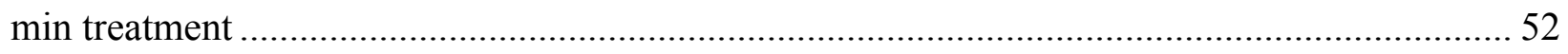
Figure 4.11. Water contact angel of a) bare PET, b) 30 min N-treatment, c) 60 min N-treatment, d) $30 \mathrm{~min}$ Ar-treatment, e) $60 \mathrm{~min}$ Ar-treatment, f) $30 \mathrm{~min}$ O-treatment, and g) $60 \mathrm{~min} \mathrm{O}$ -

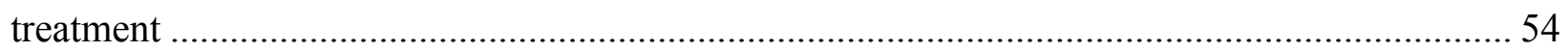

Figure 4.12. COF of untreated and ion treated PET films ........................................................... 55

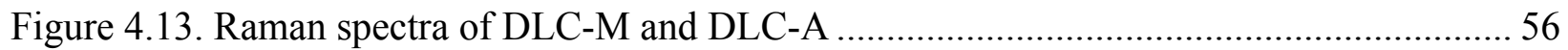

Figure 4.14. C1s XPS spectra of DLC sample deposited on PET, a) DLC-M, b) DLC-A........... 57

Figure 4.15. Water contact measurements of a) bare PET, b) DLC-M, and c) DLC-A ................ 58

Figure 4.16. Width measurement of scratch line a) DLC-M+30 min N-treatment, b) DLC-M+30 min O-treatment, c) DLC-M+60 min O-treatment, d) DLC-M+60 min N treatment, e) DLC-

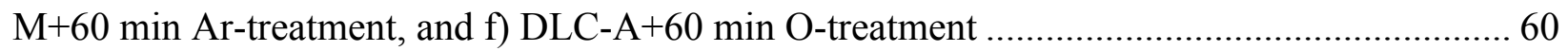

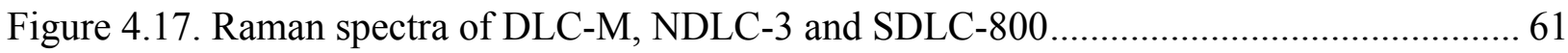


Figure 4.18. a) ATR-FTIR Spectra of bare PET, DLC-M, NDLC-3 and SDLC-800 coated PET b) C-H stretching vibration mode of DLC-M. NDLC-3 and SDLC-800 on PET ...................... 62

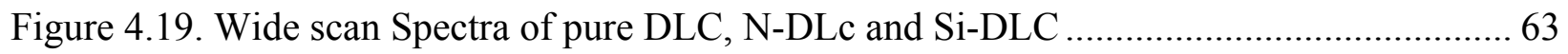

Figure 4.20. De-convolution of C1s XPS spectra of a) NDLC-3, b) NDLC-2 and c) NDLC-1 .. 64 Figure 4.21. N1s XPS spectra of Nitrogen doped DLC samples with different N concentrations65 Figure 4.22. De-convolution of C1s XPS spectra of a) SDLC-800, b) SDLC-400 ................... 66 Figure 4.23. Si2p XPS spectra of Silicon doped DLC samples with different Si concentrations 67 Figure 4.24. NEXAFS spectra of N-DLC and DLC in TEY mode ....................................... 68

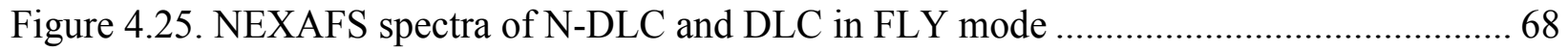

Figure 4.26. Hardness and young modulus of a) Nitrogen doped DLC, b) Silicon doped DLC

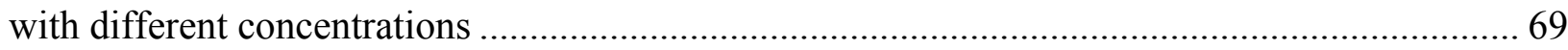

Figure 4.27. Optical microscopy of a) SDLC-800 and b) NDLC-3 after scratch testing ............ 70

Figure 4.28. COF of DLC, N-DLC and Si-DLC samples under reciprocating motion ............... 71

Figure 4.29. Roughness measurement with optical profiler of a) bare PET, b) DLC-M, c) NDLC1, d) NDLC-2, d) NDLC-3, f) SDLC-400, g) SDLC-800 ....................................................... 73

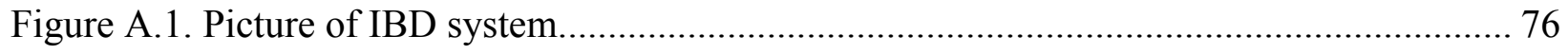

Figure A.2. A picture of Renishaw 2000 micro-Raman system ............................................ 76

Figure A.3. Picture of AFM mounted on optical microscope................................................ 77

Figure A.4. Optical profilometer (http:/www.zygo.com/met/profilers/zegageplus/zegage-plus-

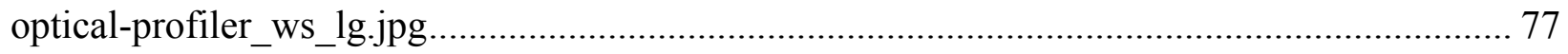

Figure A.5. Picture of Universal Material Tester designed and made by CETR...................... 78

Figure A.6. UMT during the ball-on-disk testing ............................................................ 78 


\section{ACRONYMS}

a-C:H Hydrogenated Amorphous Carbon

AFM Atomic Force Microscopy

APG Atmospheric Pressure Glow

COF Coefficient of Friction

CLS Canadian Light Source

CNT Carbon Nanotubes

CVD Chemical Vapor Deposition

DC Direct Current

DLC Diamond-Like Carbon

$\mathrm{E}_{\mathrm{p}} \quad$ Penetration Threshold

$\mathrm{E}_{\mathrm{d}} \quad$ Displacement Threshold

$\mathrm{E}_{\text {kin }} \quad$ Kinetic Energy of the Photoelectrons

$\mathrm{E}_{\mathrm{b}} \quad$ Binding Energy

EH End Hall

FLY Total Fluorescence Yield

FTIR Fourier Transform Infrared Spectroscopy

FWHM Full width at Half Maximum

$\mathrm{h}_{\mathrm{p}} \quad$ Plastic Deformation

IBD Ion Beam Deposition

IP Ionization Potential

LB_ADSA Low Bond Axisymmetric Drop Shape Analysis Model of Drop Shape Analysis

$\mathrm{L}_{\max } \quad$ Maximum Load

MSID Mass Selected Ion Beam

N-DLC Nitrogen Doped Diamond-Like Carbon

NEXAFS Near Edge X-Ray Absorption Fine Structure Spectroscopy

PE Polyethylene

PET Polyethylene Terephthalate

PECVD Plasma Enhance Chemical Vapor Deposition

PMMA Polymethyl Methacyrlate 


$\begin{array}{ll}\text { PP } & \text { Polypropylene } \\ \text { PVD } & \text { Physical Vapor Deposition } \\ \text { RF } & \text { Radio Frequency } \\ \text { SCCM } & \text { Standard Cubic Centimeters per Minute } \\ \text { Si-DLC } & \text { Silicon Doped Diamond-Like Carbon } \\ \text { SSSC } & \text { Saskatchewan Structural Science Center } \\ \text { ta-C } & \text { Tetrahedral Amorphous Carbon } \\ \text { ta-C:H } & \text { Hydrogenated Tetrahedral Carbon } \\ \text { TEY } & \text { Total Electron Yield } \\ \text { TMS } & \text { Tetramethylsilane } \\ \text { UHMWPE } & \text { Ultra High Molecular Weight Polyethylene } \\ \text { UMT } & \text { Universal Material Tester } \\ \text { XPS } & \text { X-ray Photoemission Spectroscopy } \\ \Phi \text { work } & \text { Work Function } \\ v & \text { Poisson Ratio } \\ \gamma & \text { Surface Tension } \\ \theta & \text { Contact Angle } \\ v & \text { Frequency }\end{array}$




\section{CHAPTER 1}

\section{INTRODUCTION}

\subsection{Motivations}

Due to the unique properties of polymers such as low density, low cost, chemical inertness, high specific strength, good formability, high flexibility, and variety of compositions, forms, and structures, they have been used extensively and the applications continue to increase in the past decades. For example, they have been widely used in packaging, furniture, toys, automobiles, food and beverage containers, biomedical implants, and solar power devices. Among polymers, Polyethylene terephthalate (PET) holds diverse applications as cardiovascular implants (artificial heart valves and blood vessels), food and beverage containers, and components in solar cells [13]. PET is a semicrystalline polymer with a simple long chain belong to polyester family and possesses outstanding properties like chemical inertness, high mechanical strength, toughness, and fatigue resistance. In PET structure, aromatic ring is coupled with a short aliphatic chain of a total length of $1.09 \mathrm{~nm}$ and a molecular weight of approximately 200 [4]. PET film was developed by injection molding and extrusion in late 1950s and was mostly utilized in videos, photographic and X-ray films as well as in flexible packaging. The first oriented threedimensional PET was produced in the early 1970 s by blow molding [5]. Since then, PET applications in industry have been increased rapidly. Nevertheless, PET surface suffers drawbacks of low hardness, lack of biocompatibility, and low gas retention, significantly limiting its applications.

Surface coating is a prominent way to enhance surface characteristics with no sacrifice of bulk properties. $\mathrm{SiO}_{2}$ and $\mathrm{Si}_{3} \mathrm{~N}_{4}$ thin films were applied to PET surface to improve its gas barrier properties [6-8]. However, it is hard to coat ultra-thin dense films of oxides or nitrides or carbides on PET because of its low thermal stability. It is good that diamond-like carbon (DLC) based materials can be coated on substrates at temperatures as low as $20 \mathrm{C}^{\circ}$. In addition, DLC is hard, transparent, chemical inert, highly biocompatible, a good gas barrier, and of low friction 
coefficient, high wear and corrosion resistance [8, 9], which makes DLC an ideal coating material for modifying the surfaces of PET for applications as food and beverage containers [11], and biomedical implants [10]. However, DLC usually has high internal stress due to the ion bombardment during the deposition and has low adhesion strength to polymers because of the large difference in mechanical properties, leading to the delamination of DLC from polymers. Therefore, it is very important to develop techniques to lower the stress of DLC and to enhance the adhesion strength of DLC to polymers. Different approaches have been used to improve DLC adhesion on polymers. Plasma surface treatment on polymers is one of the most common techniques to modify the polymer surface and increase the film adhesion by rising the number of free radical on the surface before deposition [12-14]. It has been reported that alloying and doping can also reduce the DLC internal stress and consequently improve the film adhesion $[15,16]$. The effect of nitrogen doping on internal compressive stress reduction in DLC coating deposited on different substrates has been investigated $[17,18]$ and the results show that it is effective in releasing internal stress. The influence of silicon incorporation into DLC deposited on PET has been studied using plasma enhanced chemical vapor deposition in a mixture of acetylene $\left(\mathrm{C}_{2} \mathrm{H}_{2}\right)$ and tetramethylsilane (TMS) and improvement of gas barrier properties was reported accordingly [19]. Another potential way to reduce the stress is to use low energy ion beams for deposition. This technique has not been fully studied for DLC deposition.

Griddles end-Hall (EH) ion sources [20], a broad beam source, can supply large ion current (up to $5 \mathrm{~A}$ ) with the average ion beam energy of $40-210 \mathrm{eV}$ that covers the ion energy range for DLC deposition. Low ion energy is beneficial for DLC deposition on PET because lower ion energy results in lower $\mathrm{sp}^{3}$ concentration with lower intrinsic stress for improved adhesion. Therefore, EH ion beam deposition would be the most suitable technique for large area DLC deposition on polymers for industrial applications [21,22]. Although progress has been made on $\mathrm{EH}$ ion beam deposited carbon thin films, investigations of the adhesion, microstructure and mechanical properties of a-C:H thin films deposited on plasma treated PET with EH ion beam are still scarcely reported. Furthermore, no report has been found on the effect of nitrogen and silicon doping on the adhesion, structure and properties of DLC on PET using EH ion beam deposition.

In this study, we report on the deposition and characterization of highly adherent DLC based thin films on PET and the investigation of the effect of doping ( $\mathrm{N}$ and $\mathrm{Si}$ ) and substrate pretreatment 
with the use of EH ion beam system on the structure and properties of DLC on PET. In addition, two different precursors $\left(\mathrm{CH}_{4}\right.$ and $\left.\mathrm{C}_{2} \mathrm{H}_{2}\right)$ were used for DLC deposition in order to compare adhesion strength of deposited films.

\subsection{Objectives}

The overall goal of this MSc research is to obtain adherent DLC thin films on polyester to enhance its surface properties by combining doping and plasma pretreatment. Specifically, the project has following objectives:

1- Enhance interfacial adhesion by plasma pretreat of polyester surface in different gas mixtures.

2- Reduce internal stress of DLC by optimizing deposition parameters and doping of $\mathrm{N}$ and Si. .

3- Evaluate the tribological behavior of the coated polyester and understand the factors affecting the tribological behavior.

4- Optimize the composition and pretreatment conditions to achieve adherent DLC films on polyester to achieve low friction and high wear resistance.

\subsection{Thesis Organization}

The present thesis consists of five chapters.

The main focus of chapter 1 is on research motivation and objectives of the present work.

Chapter 2 provides a detailed review about DLC structure, growth mechanism and deposition techniques. In addition, adhesion problem of DLC deposition on polymer is discussed and possible methods to overcome the adhesion problem is reviewed.

Detailed experimental methods including thin film deposition, ion surface treatment, structural characterization and property characterization of thin films are described in Chapter 3.

Chapter 4 is divided into three sections. In the first section, effect of ion surface treatment of PET is studied while section two reports the adhesion properties of DLC deposited on ion treated polymers. Section three states the effect of doping on structure, property, and adhesion of DLC on PET substrate.

Conclusions and recommendation for future work are provided in Chapter 5. 


\subsection{Publications}

The research conducted in this thesis resulted in preparation of two ISI journal papers. One of the papers with the title of "Fabrication and Characterization of Adherent Diamond-Like Carbon Based Thin Films on Polyethylene Terephthalate by End Hall Ion Beam Deposition" is published in Journal of Surface and Coating Technology and the other paper with the title of "The Effect of Plasma Surface Treatment on Adhesion Strength of Diamond-like Carbon Film Coated on PET" is still under preparation. In addition, this thesis work is presented in two national and international conferences as follows;

- International Conference on Metallurgical Coatings and Thin Films, San Diego, USA, April 2016

- Canadian Materials Science Conference, Hamilton, Canada, June 2016. 


\section{CHAPTER 2}

\section{LITERATURE REVIEW}

\subsection{Carbon materials}

Carbon is one of the most interesting elements that is the main composition of living beings and most of the chemical substances and has the largest number of allotropies. Carbon also ranks as the second most prominent material after oxygen (65\%), and composes $18 \%$ of the human body. Carbon can be found in a wide range of materials. These materials range from diamonds that have super high hardness and extremely high thermal conductivity, to graphite, which is very soft and can be used as a solid lubricant. Carbon has the ability to be in three different electronic configurations, $\mathrm{sp}^{1}, \mathrm{sp}^{2}$ and $\mathrm{sp}^{3}$, which is the source of its unique behaviors [16]. Carbon has six electrons, which are configured as $1 \mathrm{~s}^{2} 2 \mathrm{~s}^{2} 2 \mathrm{p}^{2}$ and illustrated in Figure 2.1.

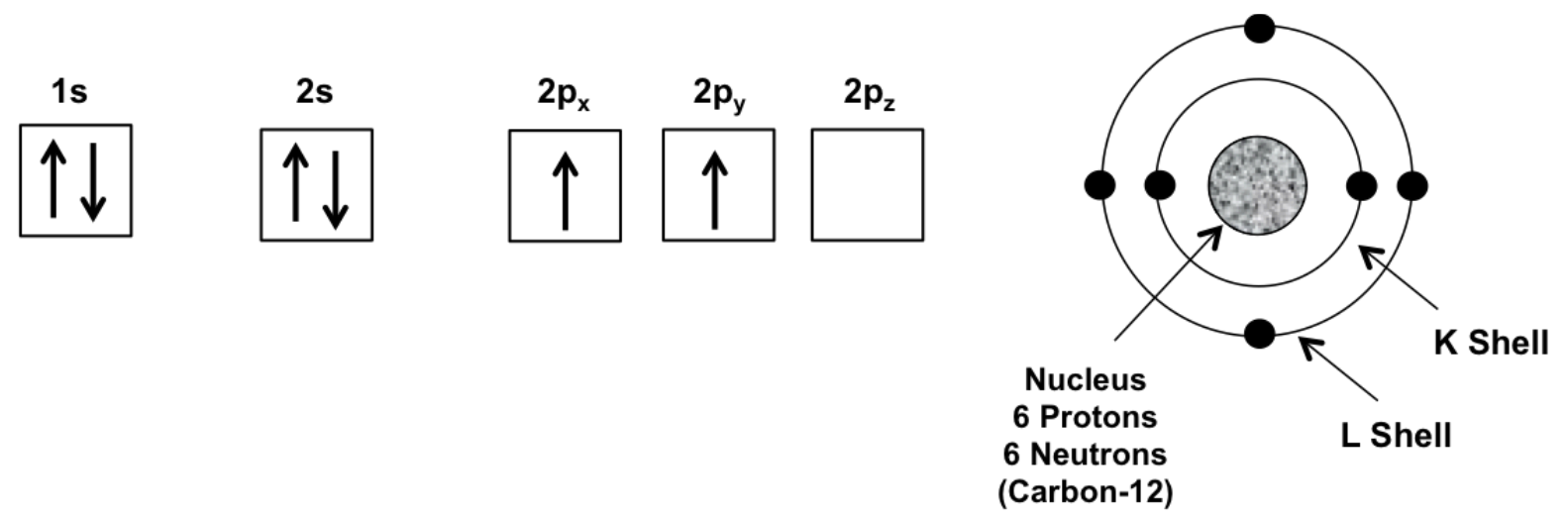

Figure 2.1. Distribution of electrons in carbon orbitals [23]

The electron distribution of carbon makes it possible to form different allotropes with three bonding configurations, as well as the ability to form single, double and triple bonds. Figure 2.2 shows different configurations of carbon atoms. In $\mathrm{sp}^{3}$, the s orbital and the three $\mathrm{p}$ orbitals are hybridized to form four equivalent sp orbitals with an equal angle of $109.28^{\circ}$ as shown in Figure 2.2 and each valence electron occupied one orbital as shown in Figure 2. 3. In this case, four of six carbon electrons in one atom contribute in bonding and as a result can form four strong 
covalent sigma $(\sigma)$ bonds with four neighbor atoms. Diamond contains metastable tetragonal sigma bonds with $\mathrm{sp}^{3}$ hybridization and possesses extreme hardness, high thermal conductivity, large band gap, high atomic density and low wear rate that makes it appropriate for tools or wear resistant components.
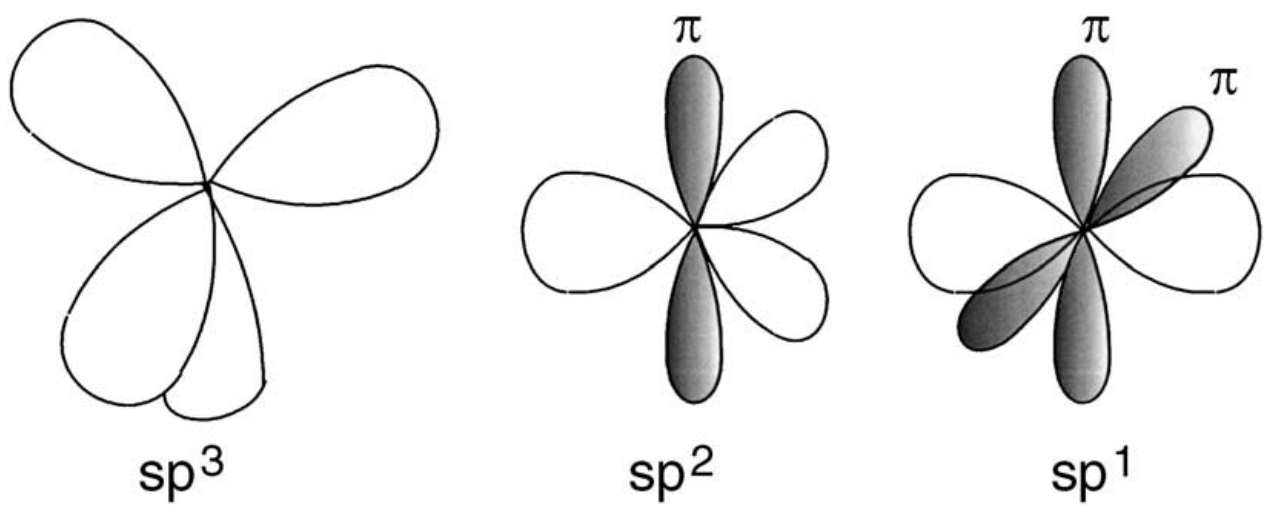

Figure 2.2. Bonding hybridizations for carbon atoms [16]

\begin{tabular}{|c|c|c|c|c|}
\hline $1 \mathrm{~s}$ & $2 \mathrm{sp}^{3}$ & $2 \mathrm{sp}^{3}$ & $2 \mathrm{sp}^{3}$ & $2 \mathrm{sp}^{3}$ \\
\hline$\uparrow \downarrow$ & $\downarrow$ & $\downarrow$ & $\downarrow$ & $\downarrow$ \\
\hline
\end{tabular}

Figure 2. 3. $\mathrm{sp}^{3}$ hybrid orbitals

In $\mathrm{sp}^{2}$ hybridization, like in graphite, one $\mathrm{s}$ orbital and two $\mathrm{p}$ orbital are hybridized to three $\mathrm{sp}$ orbitals and one orbital is left behind as p orbital. Consequently, three valance electrons fill the three sp orbitals respectively and one valence electron fills the delocalized $2 p$ orbital as demonstrated in Figure 2.4. In this case, the three identical $\mathrm{sp}^{2}$ orbitals fall in the same plane with $120^{\circ}$ angle from each other. Accordingly, the other $\mathrm{p}$ orbital that is not hybridized is perpendicularly joined to the plane and form $\pi$ bond with a neighbor atom. The sigma bonds in the $\mathrm{sp}^{2}$ hybrid possess even higher energy and shorter band length than the $\mathrm{sp}^{3}$ hybrid bonds. 


\begin{tabular}{|c|c|c|c|c|}
\hline $1 \mathrm{~s}$ & $2 \mathrm{sp}^{2}$ & $2 \mathrm{sp}^{2}$ & $2 \mathrm{sp}^{2}$ & $2 \mathrm{p}$ \\
\hline$\uparrow \downarrow$ & $\downarrow$ & $\downarrow$ & $\downarrow$ & $\begin{array}{c}\text { Free } \\
\text { delocalized } \\
\text { electron }\end{array}$ \\
\hline
\end{tabular}

Figure 2.4. $\mathrm{Sp}^{2}$ hybrid orbital

Unlike diamond, graphite materials are soft, conductive, and optically opaque because of the weak van der Waals bonds between its atomic layers. They have been used as electric conductors, lubricants, electrodes, and pencils. The other hybridization of carbon is the sp, which forms linear structure. In this case, two sp orbitals form sigma $(\sigma)$ bonds with an angle of $180^{\circ}$ in between, and the two other unhybridized $2 \mathrm{p}$ electrons can form pi $(\pi)$ bonds.

Moreover, carbon can be found as various nanostructures, e.g. Buckminsterfullerene (C60), carbon nanotubes, graphene, or amorphous structure. As is indicated by Figure 2.5, C60 has a soccer ball shape, and consists of sixty carbon atoms. It consists of covalently bonded carbon atoms in hexagonal and pentagonal rings and can be used in photovoltaic solar cells since its optical absorption increases with the increase of light intensity. A single wall carbon nanotube (CNT) consists of one-atom-thick graphite sheet with $\mathrm{sp}^{2}$ bonding hybridization rolled into a cylindrical nanostructure. Along axial direction, CNTs have extremely high strength and good thermal conductivity. Amorphous carbon is another form of carbon that contains a random network of carbon atoms with different hybridizations, which can be graphite-like or diamondlike depends on the fraction of $\mathrm{sp}^{2}$ and $\mathrm{sp}^{3}$ hybrids. Graphite-like carbon forms when the amorphous structure contains high amount of $\mathrm{sp}^{2}$ bonds. Meanwhile, diamond-like carbon consists of mostly $\mathrm{sp}^{3}$ in its structure [24]. 


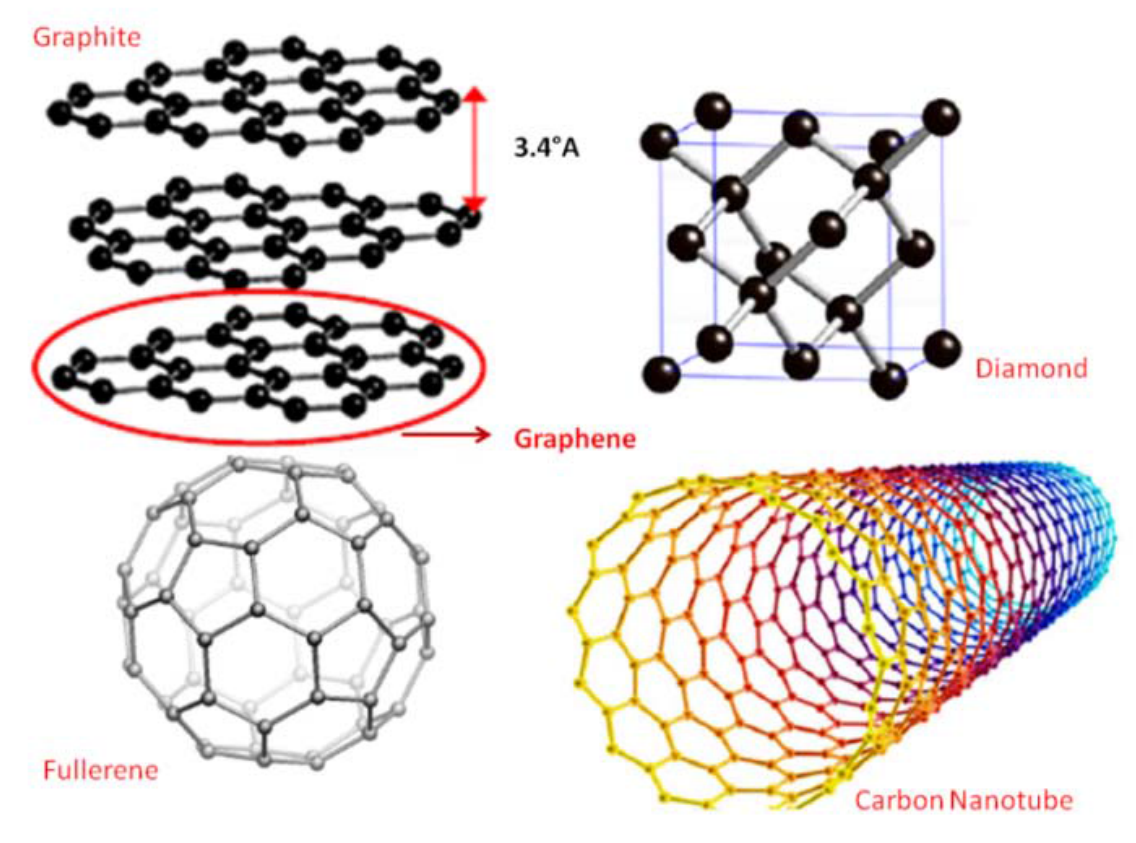

Figure 2.5. Atomic configurations of some carbon allotropes [25].

\subsection{Diamond like carbon coatings}

A diamond like carbon (DLC) coating is an amorphous carbon-based thin film that contains a significant fraction of $\mathrm{sp}^{3}$ carbon bonds in its structure. DLC can be classified in different categories based on hydrogen content and the percentage of $\mathrm{sp}^{3}$ carbon bonds. Two major categories of DLC are hydrogenated DLC and hydrogen-free DLC. Hydrogenated DLC with a moderate amount of $\mathrm{sp}^{3}$ bonded carbon and 10 to $40 \%$ hydrogen content is known as hydrogenated amorphous carbon $(\mathrm{a}-\mathrm{C}: \mathrm{H})$. By increasing the $\mathrm{sp}^{3}$ content and decreasing the hydrogen content, hydrogenated tetrahedral carbon (ta-C:H) could be formed. Hydrogen-free DLC can be amorphous carbon (a-C ) with moderate amount of $\mathrm{sp}^{3}$ and tetrahedral amorphous carbon (ta-C) with high percentage of $\mathrm{sp}^{3}$ up to $90 \%$. Different types of DLC can be illustrated in a ternary phase diagram as displayed in Figure 2.6.

Hydrogenated amorphous carbon is also classified based on atom number density and hydrogen content that was proposed by Angus and Hayman in 1988 [26]. As it can be deduced from Figure 2.7, a-C:H and a-C DLC films possess higher atom number density in comparison with polymers. 


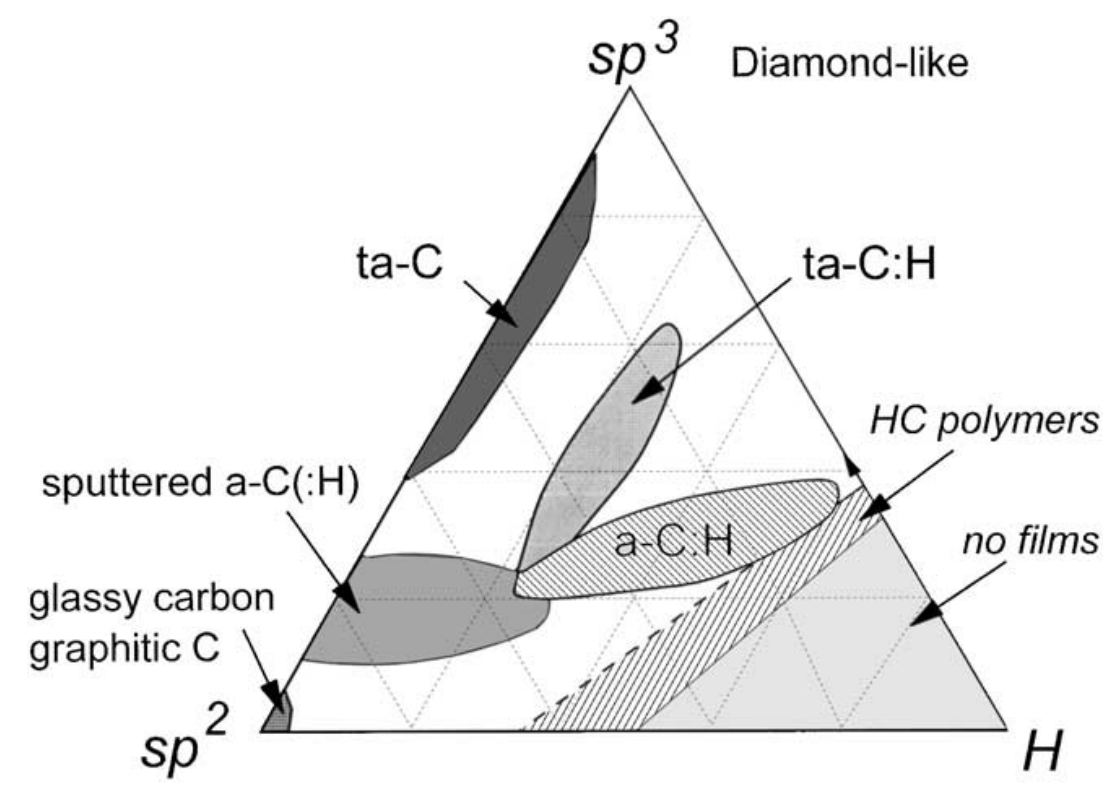

Figure 2.6. Ternary phase diagram of the C, H system [27]

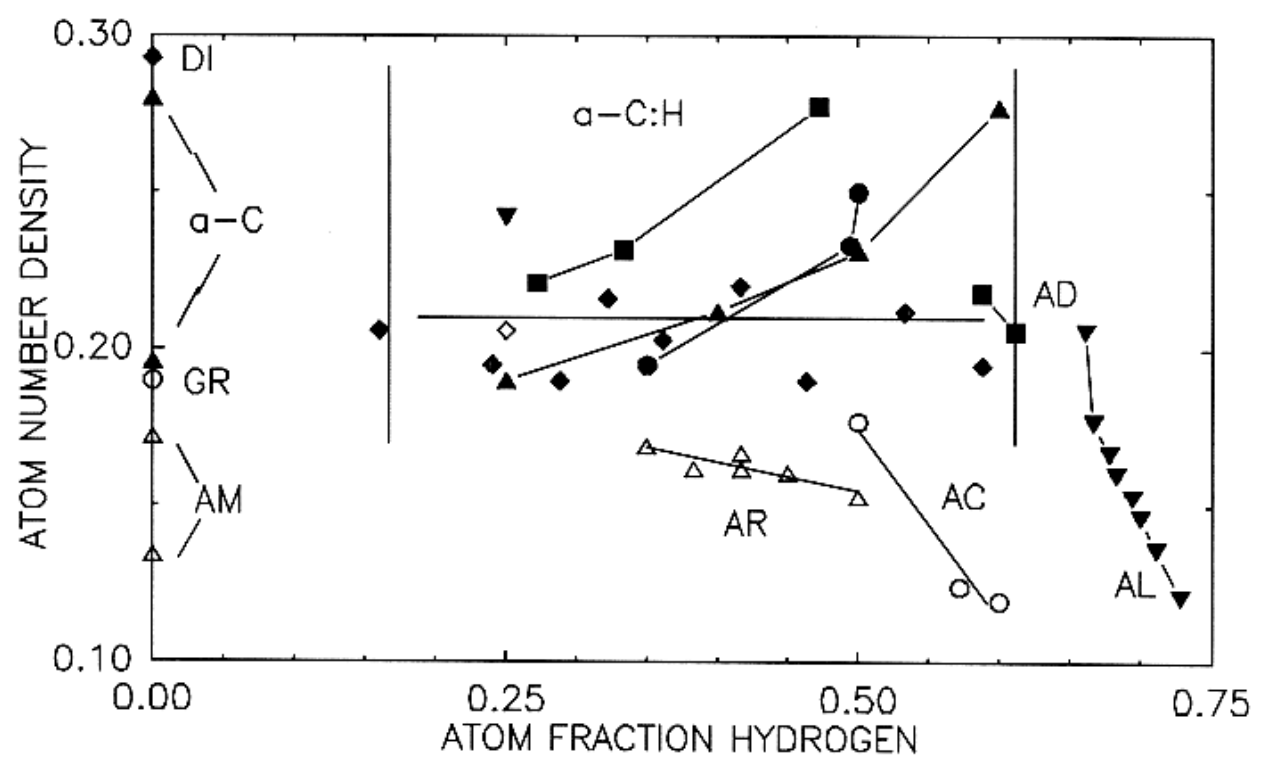

Figure 2.7. Atom number density vs. atom fraction of hydrogen [26]

(Expressed in gram atom $/ \mathrm{cm}^{3}$. Where; AC- oligomers of acetylene, AD- adamantanes, AL nalkanes, AM- amorphous carbon, AR - polynuclear aromatics, DI - diamond and GR graphite)

DLC bonding and its electronic configuration have been studied with different models. Among these models the Robertson cluster model gives a fairly basic understanding. In the Robertson 
model, the $\mathrm{sp}^{2}$ sites in planar cluster with different sizes covalently bonded with $\mathrm{sp}^{3}$ networks. The schematic of this model is shown in Figure 2.8. The arrangement of $\mathrm{sp}^{2}$ clusters determines the optical gap and electronic characteristic, while the $\mathrm{sp}^{3}$ configuration controls the mechanical properties of DLC [16]. Although several theoretical models have been developed, the detailed atomic scale structure of DLC has not been completely understood. A typical molecular dynamic simulation of a-C:H with approximately 10 at. $\% \mathrm{H}$ is shown in Figure 2.9. It details the carbon atoms with three- $\left(\mathrm{sp}^{2}\right)$ and four-fold $\left(\mathrm{sp}^{3}\right)$ atomic configuration and different kinds of hydrogen inside the structure [28].

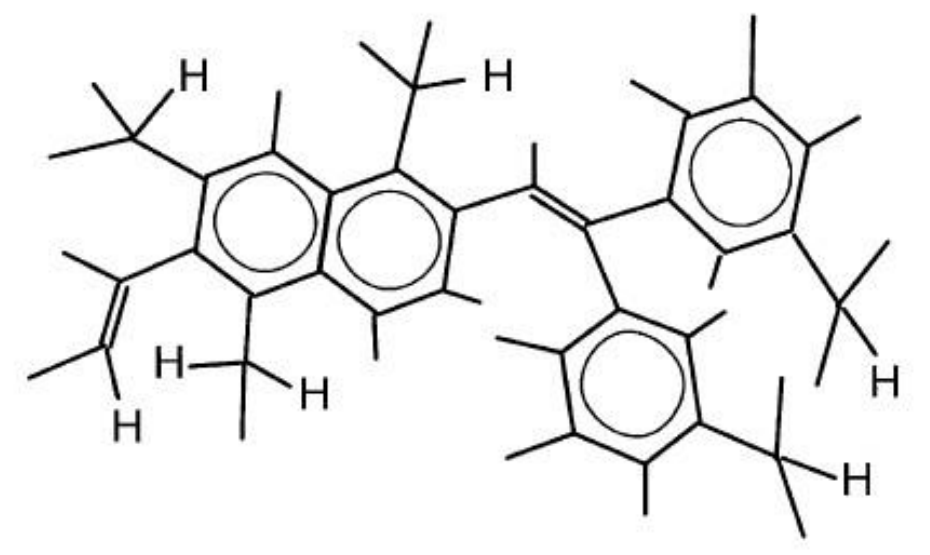

Figure 2.8. Schematic of $\mathrm{sp}^{2}$ clusters in a-C:H film [16]

Various bonding structure such as $\mathrm{sp}^{3} \mathrm{C}-\mathrm{C}, \mathrm{sp}^{2} \mathrm{C}-\mathrm{C}$, and $\mathrm{C}-\mathrm{H}$ bonds contribute to the forming of DLC amorphous structure. However, the "diamond-like" properties of DLC coatings, such as high hardness and high Young's modulus, high chemical inertness, excellent optical transparency and biocompatibility arise from its high fraction of $\mathrm{sp}^{3} \mathrm{C}-\mathrm{C}$ bonding [16, 30], which is largely dependent on the deposition methods and the processing parameters. As a result, DLC coatings have various structures and their properties vary significantly [29]. The structure, composition, and properties of various a-C and a-C:H films are summarized in Table 2.1. 


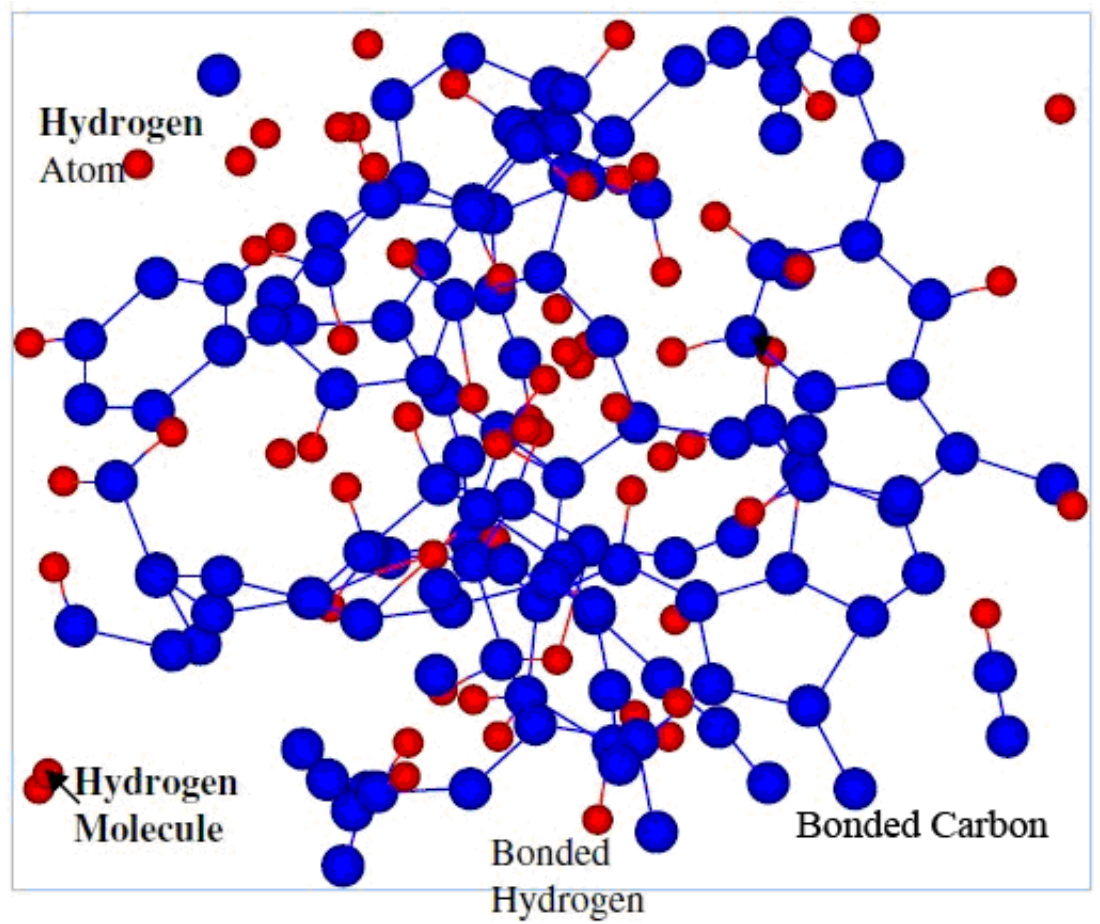

Figure 2.9. Molecular dynamic simulation of a-C:H film atomic structure [28]

Table 2.1. List of DLC structure, composition and properties [30]

\begin{tabular}{lcc}
\hline \multicolumn{1}{c}{ Variable } & a-C & a-C:H \\
\hline Hydrogen content, at \% & $<5$ & $20-60$ \\
$\mathrm{sp}^{3}, \%$ & $5-90$ & $20-65$ \\
Density, g/cm ${ }^{3}$ & $1.9-3.0$ & $0.9-2.2$ \\
Thermal stability, ${ }^{\circ} \mathrm{C}$ & $<600$ & $<400$ \\
Optical gap, eV & $0.4-1.5$ & $0.8-4.0$ \\
Electrical resistivity, ohm/cm & $102-1016$ & --- \\
Refraction index & $1.8-2.4$ & --- \\
Compressive stress, GPa & $0.5-5$ & --- \\
Hardness, GPa & $<80$ & $<60$ \\
Young's Modulus, GPa & $<900$ & $<300$ \\
\hline
\end{tabular}


Compared to a-C film, a-C:H coatings are usually softer, have lower internal-stress and could be deposited in thicker thicknesses [28]. a-C:H coatings are usually deposited by utilizing a hydrocarbon source like methane $\left(\mathrm{CH}_{4}\right)$ or acetylene $\left(\mathrm{C}_{2} \mathrm{H}_{2}\right)$ [31] while a-C films are usually deposited from carbon through sputtering or laser ablation. Hydrogen's ubiquitous nature makes it impossible to form completely hydrogen free DLC. Therefore, "hydrogen free" usually means that hydrogen content in the film is less than $5 \%$ with no hydrogen precursor was involved in DLC deposition [32].

\subsection{DLC deposition methods}

In 1971, Aisenberg and Chabot synthesized the first DLC films using an ion beam deposition (IBD) technique. Since then, a wide range of chemical vapor deposition (CVD) and physical vapor deposition (PVD) methods have been established for the fabrication of DLC films to meet the demands from various applications. Direct current (DC) or radio frequency (RF) plasma enhanced CVD, IBD, pulsed laser deposition and cathodic arc deposition techniques are widely used to deposit DLC [33]. Both hydrogenated and hydrogen free DLC can be produced with these techniques by changing carbon source from pure carbon to hydrocarbon gases. Figure 2.10 depicts different DLC deposition techniques. A particular synthesis method is preferred depending on the coating application. Each of the deposition methods has its own specific characteristics. DLC film structure and properties can vary significantly depending on the deposition techniques and the deposition parameters including substrate temperature, distribution of energetic deposition particles, energy and incidence angle, and deposition rate [33].

One of the most widely used industrial techniques for DLC deposition is sputtering due to its flexibility, ease of scale up and extensive variety of sputtering materials for doping [16]. In

sputtering technique, deposition conditions can be easily controlled while it is reasonably independent from substrate geometry. In this process, carbon energetic ions that are ejected from carbon target like graphite, Glassy carbon (C), under radio frequency or direct current plasma are employed to deposit a-C thin films [33]. Magnetron sputtering, ion beam sputtering, and reactive sputtering are the main variants of sputtering techniques. By placing the magnet behind the target in the magnetron sputtering technique, deposition rate increases due to the increase of electron paths and plasma ionization degree. Nowadays, closed-field unbalanced magnetron sputtering has been widely used because super dense ultrathin DLC films can be deposited with high 
deposition rate using this technique [28]. Ion beam sputtering is another sputtering method, in which bombardment of target with argon ions generates required carbon flux for DLC deposition. Hydrogenated DLC could be synthesized in reactive sputtering by using a mixture of plasma argon and hydrogen or methane. However, in regular sputtering, the ratio of energetic ions to neutral ions is low, which causes low $\mathrm{sp}^{3}$ content and low hardness in synthesized DLC. Nevertheless, by increasing the ion fraction through biasing the substrate, DLC with relatively high $\mathrm{sp}^{3}$ content can be obtained with a relatively low deposition rate [16].

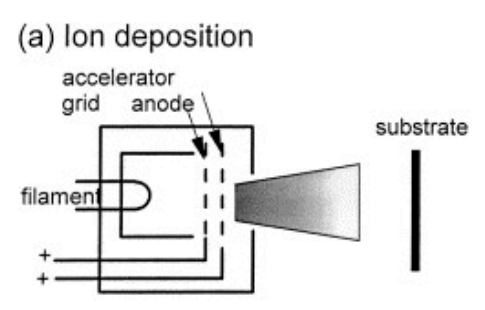

(b) Ion assisted sputtering
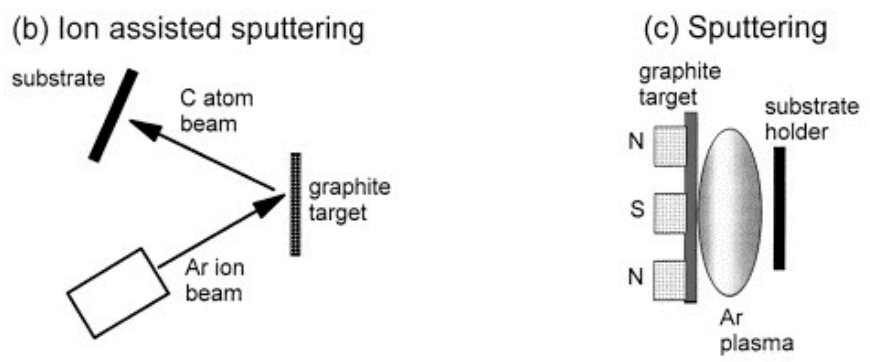

(d) Cathodic Vacuum Arc

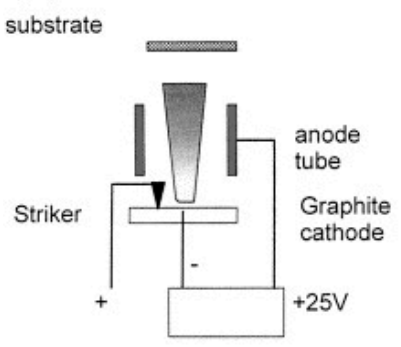

(e) Plasma deposition

(f) Pulsed laser deposition
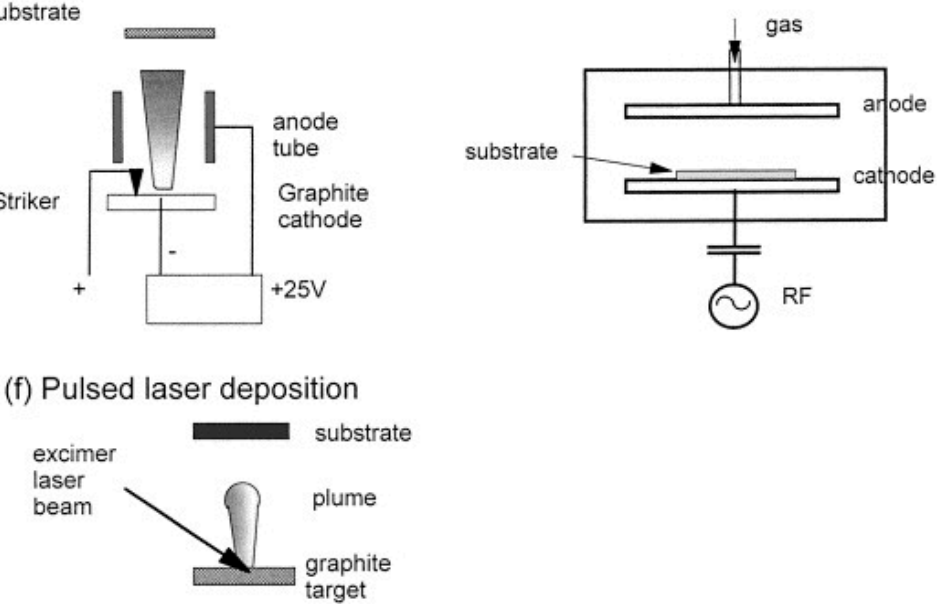

Figure 2.10. Schematic of different DLC deposition methods [16]

Ion beam deposition technique has been widely utilized to deposit DLC thin films as ion energy and ion current density can be adjusted independently [21]. In this technique, an ion beam 
generated from the precursors, either solid carbon target or hydrocarbon gas like methane, is accelerated in a vacuum environment. However, due to high amounts of unionized gas, the flux ratio of ions to the neutral species is low [16]. In ion beam deposition, the incident ions bombard on the substrate surface, penetrate the surface and enter into the subsurface layer producing high quality thin film, which is considered as ion implantation [34]. One of the advantages of ion beam techniques is their ability to use of ion implantation with other PVD techniques like sputtering or electron beam evaporation simultaneously. As a result of this combination, durable film with high adhesion can be formed on the substrate because of less built-in strain.

A special kind of ion beam technique is the Mass Selected Ion Beam (MSIB) with well-defined ion energy and well-controlled deposition condition. In this technique, generated carbon ions from graphite target possess narrow ion energy distribution. Hence, the advantage of this method is that the desirable species can be deposited while non-energetic species are filtered. Unfortunately, low deposition rate and the high cost of MSIB techniques, limit its application to laboratory scale [16].

In addition to ion beam, the arc discharge technique is well known for deposition of DLC with high purity. With the arc technique a DC or pulsed mode is used to generate electric discharge between anode and cathode source, which is carbon. The density of the generated plasma is high and the ion energy is about $30 \mathrm{ev}$. This technique is also used to dope DLC with other elements by either gas insertion or adding dopant to the cathode. Unstable cathode spot and insufficient filtering are disadvantages of this method in some applications.

Pulsed laser deposition is another effective PVD techniques to deposit DLC. It has a pulsed RF laser that is used to strike a small part of the target. Intense plasma is generated due to target vaporization under a high intensity laser pulses. Therefore, ion energy is related to laser impact on the target spot. This laboratory scale method is considered to be one of the most versatile techniques to deposit high quality DLC [16].

Plasma Enhanced Chemical Vapor Deposition (PECVD) is a widely used CVD method for DLC deposition, in which a glow discharge is used to activate gas particles. Different hydrocarbon gases used for DLC deposition in this technique are methane, ethane, acetylene, benzene, ethylene and propane.

In this work, EH ion beam system was used for DLC deposition due to availability, and DLC thin films with high quality were produced. 


\subsection{DLC deposition mechanism}

Unique characteristics of DLC come from a high fraction of metastable $\mathrm{sp}^{3} \mathrm{C}-\mathrm{C}$ bonds. It is well known that the key parameter in formation of this bond is bombarding the surface with energetic particles during the deposition [16]. In DLC deposition, the energetic carbon ions are implanted into subsurface atomic sites, which are denoted as "subplantation". Generally, the subplantation process is defined in four different stages as the following [33]:

- Penetration of bombarding particles to subsurface layer

- Incorporation of carbon and/ or hydrogen atoms in subsurface layer inducing local stress

- Formation of pure carbon layer due to sputtering and dilution of target atoms

- Continuous growth of DLC layer as a result of successive bombardment.

For carbon ions to penetrate the surface layer of substrate, certain energy is needed. This energy is called penetration threshold, $\mathrm{E}_{\mathrm{p}}$. Nevertheless, surface atoms are strongly bonded so that minimum energy is required for ion particles to relocate an atom from its initial position and generate a stable vacancy-interstitial pair. The minimum energy that an ion requires to displace a vacancy is called displacement threshold, $\mathrm{E}_{\mathrm{d}}$. Additionally, the energy that embraces surface ions is known as binding energy, $\mathrm{E}_{\mathrm{b}}$. Accordingly, the net penetration threshold for a set of carbon ions with certain critical energy is defined by

$E_{p} \sim E_{d}-E_{b}$

An ion with lower energy than the penetration threshold will not be able to penetrate to subsurface layer, and therefore, must stay on the surface in its low energy $\mathrm{sp}^{2}$ state. However, ions with higher energy than $E_{p}$ will penetrate to the subsurface layer. Thereby it will increase the local density and consequently form $\mathrm{sp}^{3}$ bonding. By increasing the ion energy, ions will acquire enough energy to penetrate deeper into the sub-layer meanwhile a set number of ions are involved in atoms displacement, causing thermalisation and relaxation [16], thus reducing the local density and losing $\mathrm{sp}^{3}$ bonding.

In order to deposit DLC thin film with high $\mathrm{sp}^{3}$ concentration, optimum ion energy should be kept around $100 \mathrm{eV}$ as Robertson reported [27]. With this energy, carbon ions have sufficient energy to penetrate into the substrate without any effect on the growth of surface density to produce maximum $\mathrm{sp}^{3}$ bonding [35]. Other researchers have also proposed that regardless of the 
deposition technique, high $\mathrm{sp}^{3}$ concentration in such an ion-dominated process like DLC deposition could be achieved with approximate ion energy of $100 \mathrm{eV}$ [28].

\subsubsection{Growth of a-C:H films:}

Although hydrogenated DLC follows the basics of sub-plantation model, the growth mechanism is slightly more complex than a-C films. With the deposition of hydrogenated amorphous carbon films, different precursors such as benzene, methane, ethane, propane and acetylene can be utilized. Distribution of ion energy plays an important role in growth of a-C:H thin films. The growth mechanism of hydrogenated carbon films is divided into three major steps:

- Dissociation and ionization of precursor gas

- Reaction of plasma with the surface of growing thin film

- Reaction in the subsurface later of film.

For studying the complex growth model of a-C:H, a thorough understanding of the chemical process of neutral species, dehydrogenation, as well as the physical process of sub-plantation is required. Under a plasma environment, ions and neutral species are generated that contribute in film growth. Neutral species in plasma contain un-ionized precursor gas, mono-radicals such as $\mathrm{CH}_{3}$, di-radicals and other unsaturated species such as $\mathrm{C}_{2} \mathrm{H}_{4}$ or $\mathrm{C}_{2} \mathrm{H}_{2}$. Usually the growth rate is independent from temperature but it can affect etching and consequently decrease the total growth. Since the surface of hydrogenated DLC is covered with C-H bonds, di-radicals along with other unsaturated species can easily absorb onto the surface and react with the film. Contribution of mono radicals in film growth is less than di-radicals since they only react with film if there is any dangling bond.

The role of hydrogen in the growth mechanism of a-C:H film is very significant because it has a small radical size and weak interaction with carbon atoms that makes it easy for hydrogen atom to penetrate deeper in the film. Consequently, hydrogen would bond with other hydrogen atoms and form hydrogen molecules, leaving unsaturated dangling bonds on the subsurface. The entire process of a-C:H growth involves the chemical procedures of the different species in the plasma, dehydrogenation-recombination, and sub-plantation of the energetic ions. Figure 2.11 present the growth model of a-C:H thin film which is suggested by Robertson [27]. 


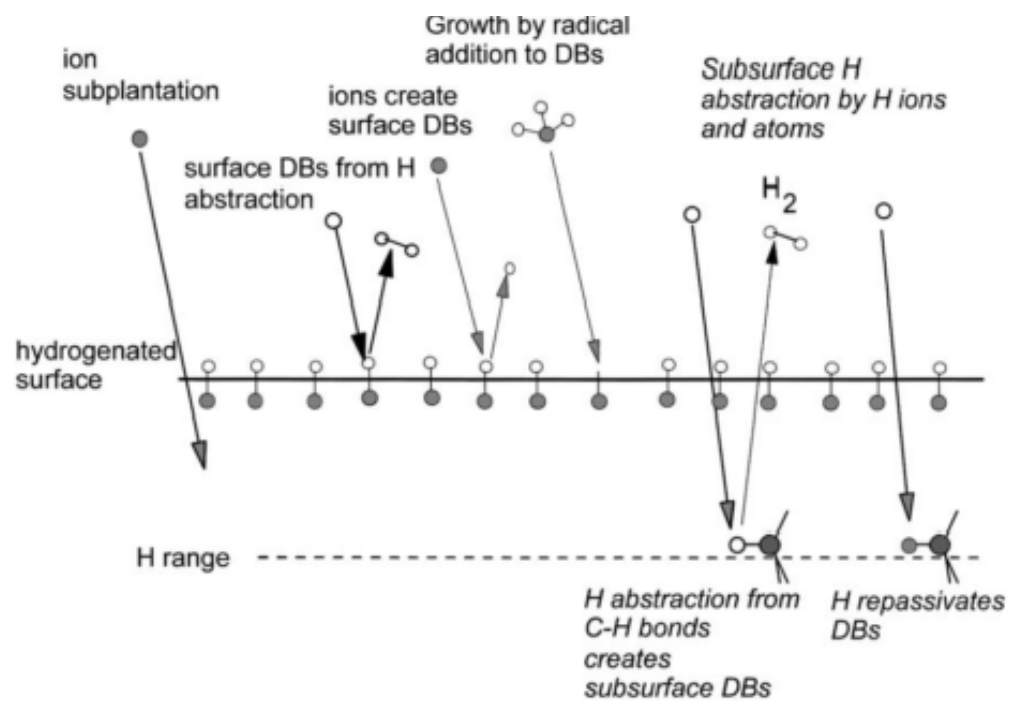

Figure 2.11. Model of growth process in a:C-H [16]

\subsection{Diamond-like carbon thin films on Polyethylene terephthalate (PET)}

Polyethylene terephthalate (PET) is semicrystalline polymer with a simple long chain. It belongs to polyester family, which possesses outstanding properties including chemical inertness, high mechanical strength, toughness and fatigue resistance. PET film was developed by injection molding and extrusion in late 1950s and was mostly utilized in video, photographic and X-ray films, as well as uses in flexible packaging. In the development of PET production, oriented three-dimensional PET was first produced in the early 1970s by blow molding. Since then, PET application in the plastic industry increased rapidly [5].

Repeating units are the major structure of PET polymer as it can be seen in Figure 2.12. In this structure, an aromatic ring is coupled with a short aliphatic chain with the total length of $1.09 \mathrm{~nm}$ and molecular weight of 200 [4]. In comparison with other polymers like polyolefin or polyamide, PET is stiff with fairly high thermal stability due to its structure. PET crystalline melting temperature is around $260^{\circ} \mathrm{C}$.

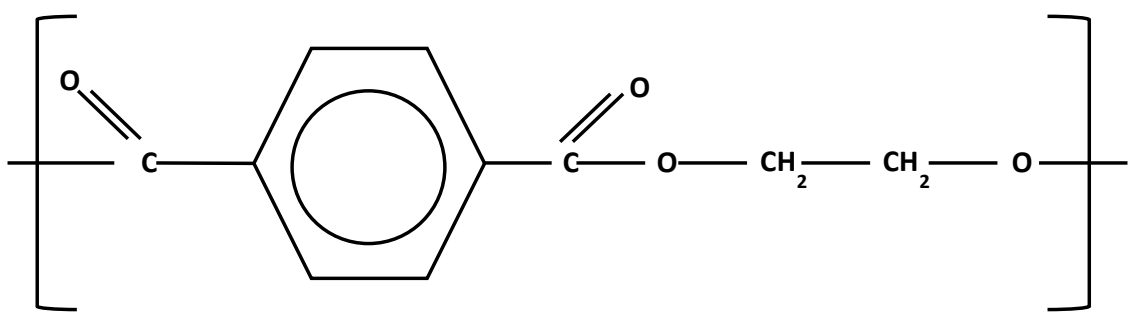

Figure 2.12. Polyethylene terephthalate (PET) structure 
PET can be implemented in cardiovascular implants such as artificial heart valves and blood vessels [1]. In addition, researchers found that PET can be a potential replacement for glass and silicon in solar constructions. Another application of PET is in food and beverage containers. Since PET, in comparison with other plastics, is remarkably well balanced in terms of required properties like strength, gas retention, flavor retention, and economy [2,3]. Gas permeation is the main drawback of polymers in such applications that can significantly affect the food quality. In order to overcome the gas barrier property of polymers, different techniques such as multilayer, coating, scavenging agents, and composite fortification have been employed. Among them, surface-coating techniques, like DLC and silicon oxide $\left(\mathrm{SiO}_{\mathrm{x}}\right)$ produced by plasma environment, could be one of the most effective methods whereby only a thin layer of material could greatly enhance gas permeation [3]. The gas barrier properties of PET have been investigated and can be improved by 20-30 times by deposition of $\sim 50 \mathrm{~nm}$ DLC film [36]. Although other oxide materials such as $\mathrm{Al}_{2} \mathrm{O}_{3}$ and $\mathrm{SiO}_{2}$ films can be used for this purpose, recyclability and biocompatibility of DLC makes it the best choice for food packaging industry $[2,3,37]$.

Tsubone et al. [12] studied on the oxygen permeation of DLC deposited on semicrystalline PET, polyethylene (PE) and polypropylene (PP) polymers, and amorphous polymethyl methacyrlate (PMMA) polymer. They reported that the gas barrier property of DLC coated samples dramatically improved after DLC deposition. They also measured gas barrier property of fractured DLC by mechanical deformation on substrate. They found that by increasing the number of cracks and lower residual strains on substrate like PET, gas barrier properties are improved in comparison with lower number of cracks on substrate and higher residual strains.

Kodama et al. [37] also fabricated the high gas barrier carbon films on PET substrate, which was fabricated in an atmospheric pressure glow (APG) plasma CVD equipment using an acetylene process gas. They proved that coated substrates have higher gas barrier property compared with those of uncoated PET substrates.

Tashiro et al. [3] also improved the gas barrier property of different polymers by DLC coating deposition and organosilane interlayer. They fabricated organosilane interlayer by immersing the substrate in a silane-coupling agent as a precursor before DLC deposition. They discovered that the cause of decreasing in oxygen transition rate was fabrication of highly adherent, flawless DLC coating on polymer substrates after treatment. 
Abbas et al. [19,38] studied the gas barrier property of PET after deposition of hydrogen free DLC, hydrogenated DLC and silicon doped hydrogenated DLC. They realized that the gas permeation of silicon doped DLC was higher than that of hydrogenated and hydrogen free DLC coatings. They mentioned that, firstly, it could be due to the incorporation of hydrogen, thus increasing the polymer-like bonds in the coating. Secondly, due to replacing strong C-C bonds with C-Si bonds, it causes relaxation of residual stress in the coating.

\subsection{Adhesion improvement of DLC on PET substrate}

Surface coating is a prominent way to enhance surface characteristics without sacrificing bulk properties. Diamond-like carbon (DLC) is hard, transparent, chemical inert, highly biocompatible, of a good gas barrier, and of low friction coefficient, high wear and corrosion resistance $[8,9]$. It is also an ideal coating material for modifying the surfaces of polymers for applications such as food and beverage containers [11], biomedical implants, and solar reflectors. However, DLC usually has high internal stress due to the ion bombardment during the deposition and has low adhesion strength to polymers because of their large difference in mechanical properties, leading to the delamination of DLC from polymers. Therefore, it is very important to develop techniques to lower the stress of DLC and to enhance the adhesion strength of DLC to polymers. One way to improve adhesion of DLC and PET is doping DLC with other elements like nitrogen and silicon. Another effective way is polymer surface modification techniques by ion surface treatment. Formation of free radicals on the polymer surface as well as new chemical bonds could cause improvement of adhesion between DLC and polymer. Therefore, doping and alloying of DLC and plasma surface treatment will be discussed in the following sections.

\subsubsection{Doping and alloying of DLC}

One of the important categories of diamond-like carbon is achieved by incorporation of different elements in the coating structure, resulting in obtaining multifunctional DLC with improved properties over pure DLC. In order to deposit DLC with desirable properties for specific applications and maintain their amorphous phase, controlling of content, distribution and the nature of dopants is very critical. Different dopants such as light elements (B, Si, N, O or F), metals (Ti, Co, W, Ni), or their combinations are used to improve DLC properties such as hardness, friction and wear resistance, electrical conductivity, thermal stability and 
biocompatibility. Doping can also enhance the film adhesion and decrease the internal stress due to ion bombardment during deposition. Figure 2.13 schematically shows the effects of different dopants on DLC film properties.

\subsubsection{Metal doped DLC}

Different metals including $\mathrm{Ti}, \mathrm{Ta}, \mathrm{Cr}, \mathrm{Ni}, \mathrm{Al}, \mathrm{Cu}, \mathrm{Ag}$, etc., have been used in different deposition techniques such as biased target ion beam deposition [39,40], reactive sputtering [41] cathodic arc evaporation [42], ion implantation [43], and dual plasma deposition [44] magnetron sputtering [45] to deposit metal doped DLC. The structure of the film after doping with metals has not been completely understood, but researchers believe that either pure metals or metal carbide could disperse in DLC with nanocrystalline structure. Reducing compressive internal stress due to ion bombardment and enhancement of adhesion of thin film to substrate are the known advantages of metal incorporation in DLC. In addition, by forming metal carbide in a carbon matrix, chemical erosion by hydrogen bombardment can be reduced and the deposition rate can be increased.

Studying DLC properties after doping with different elements is one of the interesting areas for researchers around the world. Incorporation of nanocrystalline chromium into DLC network could significantly improve wear resistance of the coating [46] while formation of titanium carbide in a carbon matrix could cause adhesion enhancement, thermal stability and outstanding mechanical properties [47]. Doping DLC with Nickel could also increase electrical conductivity, reduce coefficient of friction, and enhance wear resistance and form great adhesion to substrate because of strong bonds [48]. No degradation and oxidation has been found in Mo and W doped DLC films after annealing in $500{ }^{\circ} \mathrm{C}$ [44]. Therefore, in comparison with pure DLC, metal doped DLC offers higher hardness and elastic modulus, less coefficient of friction, better wear resistance and well adhesion due to lower internal stress during film deposition $[43,49]$. 


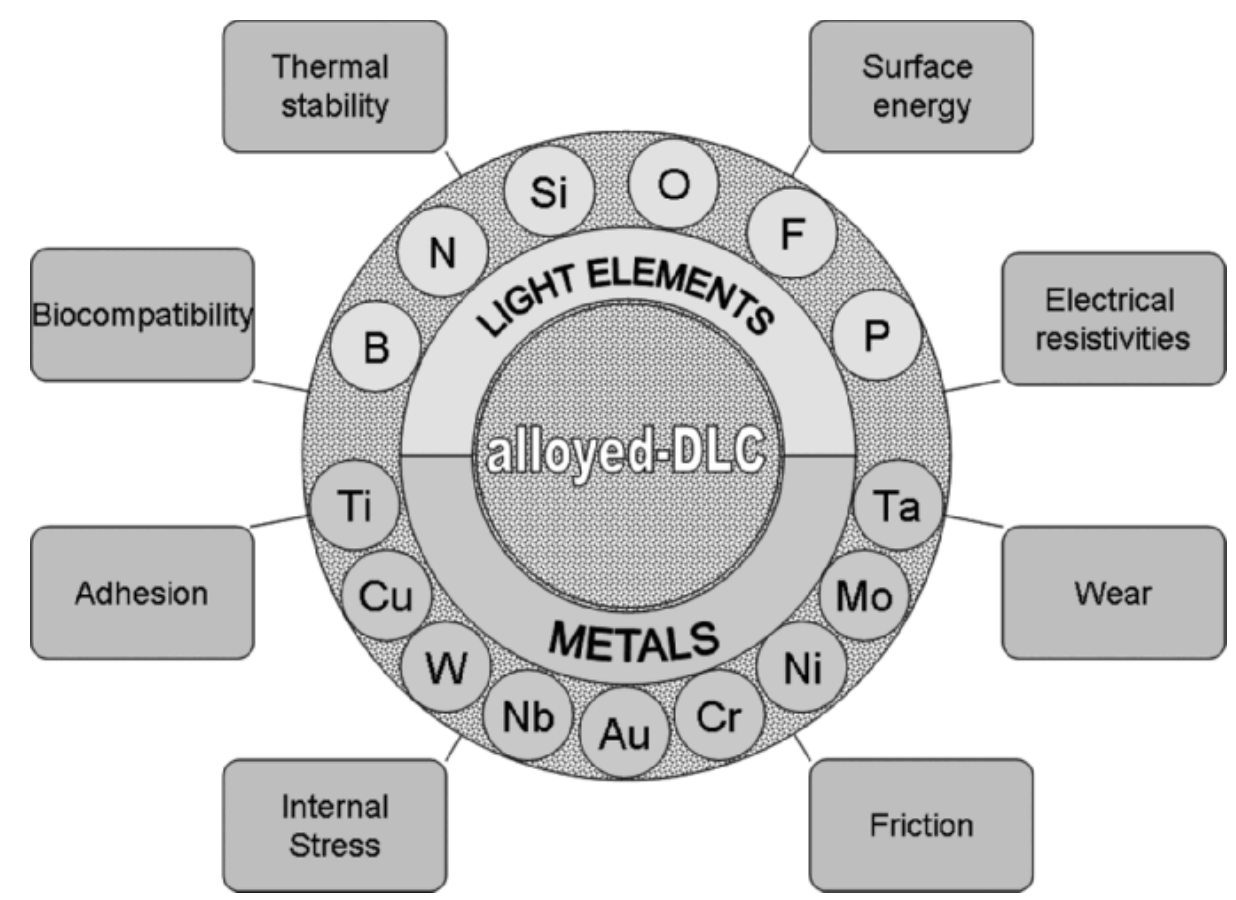

Figure 2.13. Schematic of most common doping elements in DLC for obtaining improved properties [15]

\subsubsection{Non-metal doped DLC}

Various PVD and CVD techniques like biased target ion beam deposition [50], pulsed laser deposition [51], direct current magnetron sputtering [52], filtered cathodic arc deposition [29], plasma immersion ion implantation and deposition [53], and radio frequency plasma enhanced CVD [54] have been used to deposit DLC thin films with non-metal elements such as N, B, Si, F. Low friction and wear rate under both ambient and dry nitrogen is the result of the incorporation of silicon into DLC that is mainly causes the formation of silicon oxide $\left(\mathrm{SiO}_{2}\right)$ at the surface. These properties make Si-DLC coating a perfect candidate for tribological applications [55]. In addition, it has been found that the concentration of silicon is very critical as higher $\mathrm{Si}$ concentrations in a DLC coating could cause excessive internal stress and formation of cracks in the coating [56]. Fluorine doped DLC has been studied by many researchers for several years. However, the benefit of doping DLC with fluorine is very limited because of the formation of the $-\mathrm{CF}_{2}$ and $-\mathrm{CF}_{3}$ bonds which leads to reduction of coating density and hardness as well as the formation of soft coating with high wear rate. Still, some researchers were able to deposit F-DLC with less micro-wear and lower friction by using magnetic recording media [55]. It has also been found that F-DLC could considerably enhance film antithrombogenicity for specific applications 
on blood contacting medical materials [57]. The result of studies on boron doped DLC shows that it has increased electrical conductivity and hardness through promoting $\mathrm{C}-\mathrm{C} \mathrm{sp}^{3}$ bonding [58]. Incorporation of Nitrogen into DLC makes another interesting DLC structure with improved film mechanical properties, tribological characteristics, haemocompatibility, and adhesion $[17,50]$. Nitrogenated DLC and silicon doped DLC will be discussed in details in the next section.

\subsubsection{Nitrogen doped DLC}

Nitrogen is one of the useful dopants in DLC due to its versatile properties, which gained a vast interest among scientist in the past decades. The first idea of developing nitrogen doped DLC was proposed by Liu and Cohen based on synthesizing $\beta-C_{3} \mathrm{~N}_{4}$ structure. However, formation of solid solution in nitrogen doped DLC is difficult. Nitrogen atoms can form $\mathrm{sp}^{3}$ configuration or $\mathrm{sp}^{2}$ sites due to doping with carbon as it is shown in Figure 2.14 [16]. Pyridine, pyrrole and nitrile could also form instead of solid solution.

By incorporation of nitrogen into DLC, cross-linking between the planes could be facilitated due to formation of pentagonal rings [59]. Consequently, the films with high elasticity and enhanced toughness would be produced. Nitrogen doping could promote formation of $\mathrm{sp}^{2}$ configuration due to $\mathrm{N}-\mathrm{H}, \mathrm{C}=\mathrm{N}, \mathrm{C} \equiv \mathrm{N}$ bonds formation and hindering carbon-carbon links [60]. As a result, a$\mathrm{C}: \mathrm{H}: \mathrm{N}$ would be softer than pure DLC and with excellent tribological properties [61]. In lower nitrogen content, nitrogen tends to form more $\mathrm{sp}^{2}$ bonds, whereas by increasing the nitrogen concentration more $\mathrm{sp}^{3}$ sites will be formed [62]. Thus, formation of softer DLC with lower residual stress could enhance film adhesion to the substrate [17]. Although many studies have been carried out on nitrogenated DLC due to its excellent properties, limited studies are reported using low energy end hall ion beam deposition technique. Therefore, studying the structure, properties and adhesion of N-DLC deposited on PET with the aid of E-H ion source would be beneficial.

\subsubsection{Silicon doped DLC}

Several authors have reported deposition of Si-DLC using $\mathrm{SiH}_{4}$ and hydrocarbon gases [63,64] using sputter methods [64,65] in mid-1970s and 1980s. The friction coefficient of Si-DLC was investigated by a Japanese research group in the early 1990s [66]. These researchers found that 
measured friction values were extremely lower in Si-DLC compared to a-C:H in both humid and dry atmospheres. Similar behavior was also found in vacuum conditions by Miyake et al. [67]. Furthermore, structures and properties of a-C:H:Si thin films, particularly their tribologycal properties, have been studied in the past decade [15]. A high thermal stability is another significant property of Si-DLC compared to pure DLC. Incorporation of Si into DLC could stabilize $\mathrm{sp}^{3}$ bonding configuration against graphitization [68]. It has been reported that both coefficient of friction and hardness will remain constant in Si-DLC samples even after annealing at $500{ }^{\circ} \mathrm{C}$. Meanwhile, pure DLC properties failed under $400{ }^{\circ} \mathrm{C}$ annealing [69]. The following researches suggested potential application of Si-DLC thin films. However, limited research has been conducted in this field using sputtering and hydrocarbon gas in end hall ion source and investigation of the adhesion strength of Si-DLC on PET. Thus, studying the structure-property relationship of Si-DLC using end hall ion source could be very helpful.
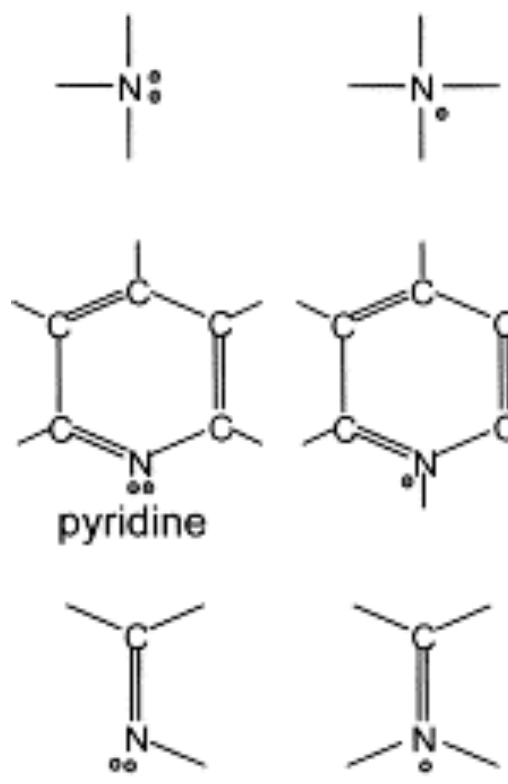
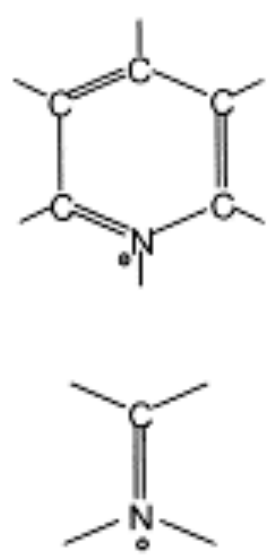
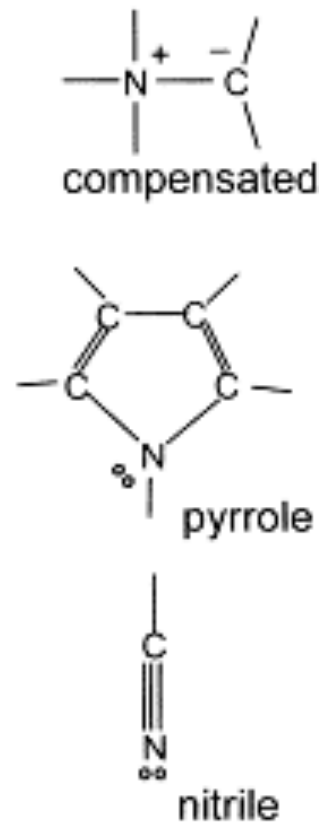

Figure 2.14. Carbon-Nitrogen bonding configurations [16]

\subsubsection{Polymer surface modification techniques}

Due to unique properties such as low density, low cost, chemical inertness, high specific strength, high ductility, good formability and high flexibility, polymers are available in a wide variety of compositions, forms, and structures. Thus their applications have increased steadily in 
the past decades. They have been widely used in packaging, furniture, toys, automobiles, food and beverage containers, biomedical implants, and solar powers. Nevertheless, they suffer from low hardness, limited strength and biocompatibility, which significantly limit their applications. Therefore, extensive research has been conducted in this field to enhance chemical and physical properties of polymer surfaces. Surface modification techniques on polymers is one of the more widely used techniques to initiate particular functional groups at the surface, enhance adhesion characteristics, rise hydrophobicity, and alter surface morphology of polymers [70,71]. Flame treatment, corona discharge, irradiation, chemical treatment, and plasma treatment are the most

utilized techniques in surface treatment of polymers. Among them, plasma treatment is a promising technique to modify polymer surfaces.

\subsubsection{Plasma surface treatment}

In this thesis, the term "plasma surface treatment" is referred to the modification technique under high vacuum with non-polymerizable gases like argon, oxygen and nitrogen. This technique is mostly used for cleaning and etching the surface, which causes formation of a functional group and cross-links at the surface of polymers due to the interaction of ions with surface top layer. The mechanism of plasma surface treatment is defined by four major phenomena [13].

(i) Cleaning of the surface (removal of some organic impurities from the surface).

(ii) Ablation or etching of the weak boundary layer from the surface and increasing the surface area for consequence treatment.

(iii) Crosslinking or branching near-surface molecules that results in strength enhancement of surface.

(iv) Modification of surface chemical structure due to bombardment of the surface with high energetic ions.

Subsequently, the synergetic combination of the above processes could affect adhesion improvement of polymer for further processing. During plasma treatment, any one of the four mechanisms could be be dominant depending on substrate, gas chemistry, reactor shape and working parameters.

Plasma treatment also has some advantages over other surface treatment methods as listed bellow:

1. Bulk properties of polymer would not be affected, as it is limited to the top surface layer. 
2. Plasma process is independent from substrate chemical reactivity or chemical structure.

3. By changing the precursor in plasma, broad range of functional groups could be initiated on the surface.

4. The modification is uniform on the entire surface.

5. Plasma surface treatment is a one-step process and consequent thin film deposition could happen in the same chamber without stopping the vacuum.

Besides the mentioned advantages, some of the disadvantages of this technique could be as follows:

1. It is a fairly expensive method because this process should be conducted in vacuum.

2. Precise control of the chemical composition of surface after modification is difficult due to the complexity of plasma processes.

\subsection{Structural characterization of diamond-like carbon thin films}

\subsubsection{Raman spectroscopy}

Inelastic scattering of monochromic light source is the basic of Raman spectroscopy. Generally, if the energies of photons change after incident to specimen, inelastic scattering of light happens (around 1 in $10^{7}$ photons), which is known as Raman scattering. Measuring the difference between incident photon energy and Raman scattering provides information of the molecule vibrations. In other words, if molecule vibrates with the characteristic frequency $v_{m}$ under incident beam with frequency of $v_{0}$, three different frequencies can be emitted as it is illustrated in Figure 2.15.

- $\boldsymbol{v}_{\boldsymbol{0}}$ is called elastic Rayleigh scattering that happens $99.999 \%$ of time. In this state, the frequency of emitted light is equal to incident beam.

- $v_{\boldsymbol{o}}-\boldsymbol{v}_{\boldsymbol{m}}$ is called Stokes frequency, meaning a fraction of molecules absorbs energy and start vibrating with frequency $v_{m}$. As a result, a photon loses its energy to a molecule and scatter with the frequency of $v_{0}-v_{m}$.

- $v_{0}+v_{m}$ is called Anti-Stroke frequency and means that photons gain some energy after incident with the molecules. In this case, the molecule is in a higher vibration state during incident and it causes the molecule to return to the ground state and emit light with $v_{0}+v_{m}$ frequency. 
Visible Raman spectroscopy is a powerful non-destructive technique to characterize carbonbased coatings such as diamond, graphite, carbon nanotubes, and DLC. This technique is widely used to analyze the detailed bonding structure of DLC. Raman works based on the energy differences between incident beam and scattered beam, which is particular for each individual substance or bond. Ultimately, Raman spectrum is the number of scattered light versus Raman shift (measured in $\mathrm{cm}^{-1}$ ). A schematic of Raman scattering of molecule is shown in Figure 2.16.

\section{Inelastic Scattering}

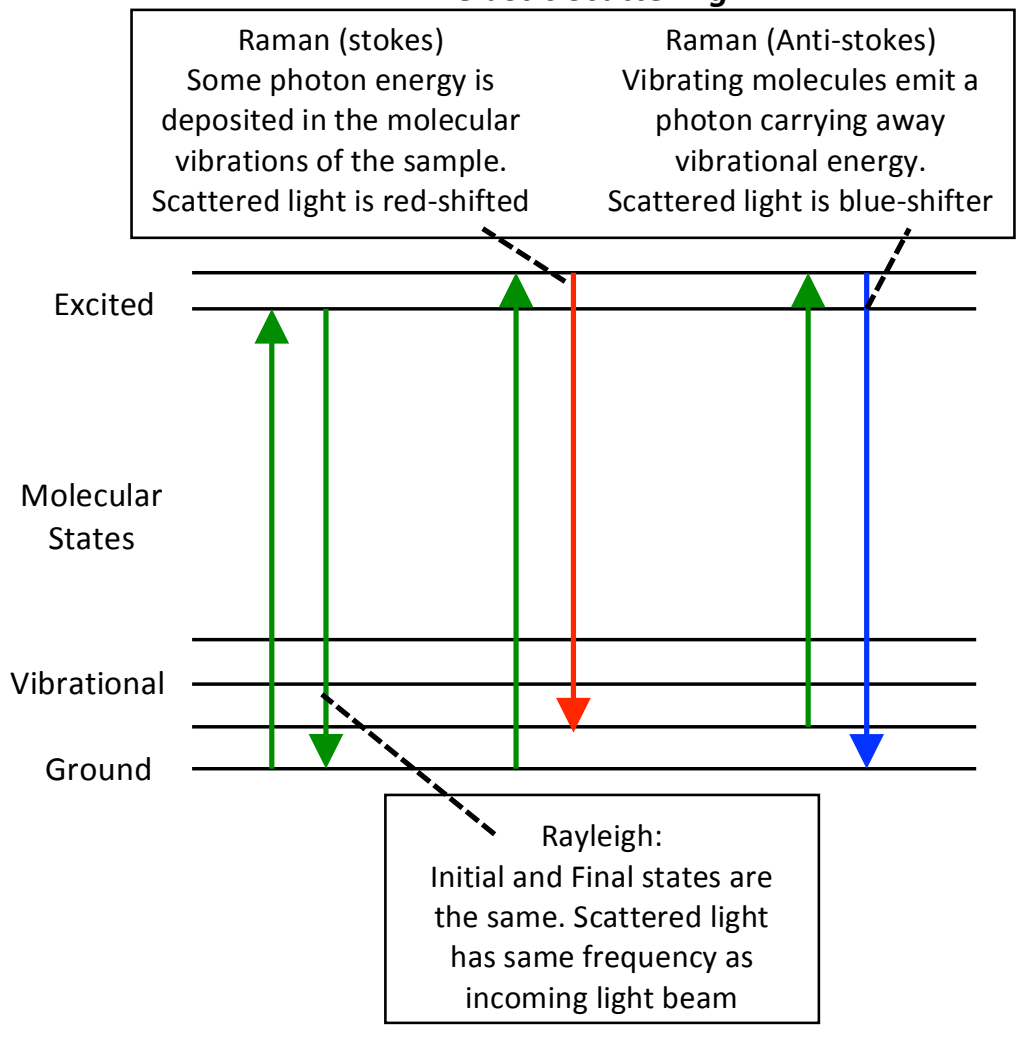

Elastic Scattering

Figure 2.15. Raman energy levels

As it is shown in Figure 2.17, carbon atoms show characteristic peaks between $800 \mathrm{~cm}^{-1}$ and $2000 \mathrm{~cm}^{-1}$ in the Raman spectra. It has been noted that Raman spectra is mainly sensitive to $\mathrm{sp}^{2}$ sites due to its higher cross sectional area. Therefore, in the case of diamond, the only peak that could be observed is at $1332 \mathrm{~cm}^{-1}$. Meanwhile, single crystal graphite shows a characteristic at $1570 \mathrm{~cm}^{-1}$, which is called " $\mathrm{G}$ " peak [61]. Raman spectra of disordered graphite shows another peak at $1360 \mathrm{~cm}^{-1}$ in addition to $\mathrm{G}$ peak, which is well known as "D" peak. Accordingly, in 
amorphous carbon Raman spectra, stretching of $\mathrm{sp}^{2}$ bonded atoms in both rings and chains is contributed to G peak located around 1360 while $\mathrm{D}$ peak is contributed to breathing modes of $\mathrm{sp}^{2}$ atoms in the rings located around 1575 [72].
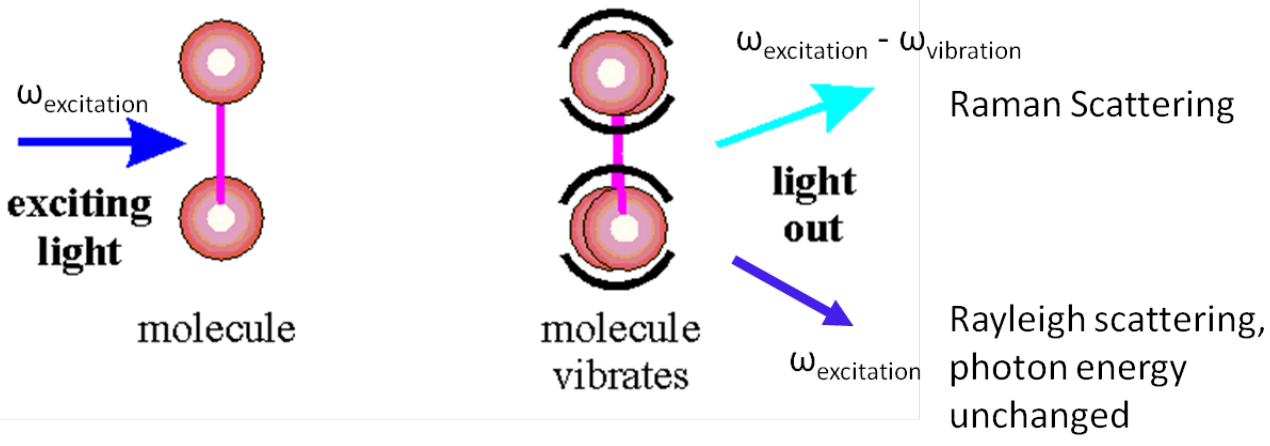

Figure 2.16. Raman Scattering from a molecule

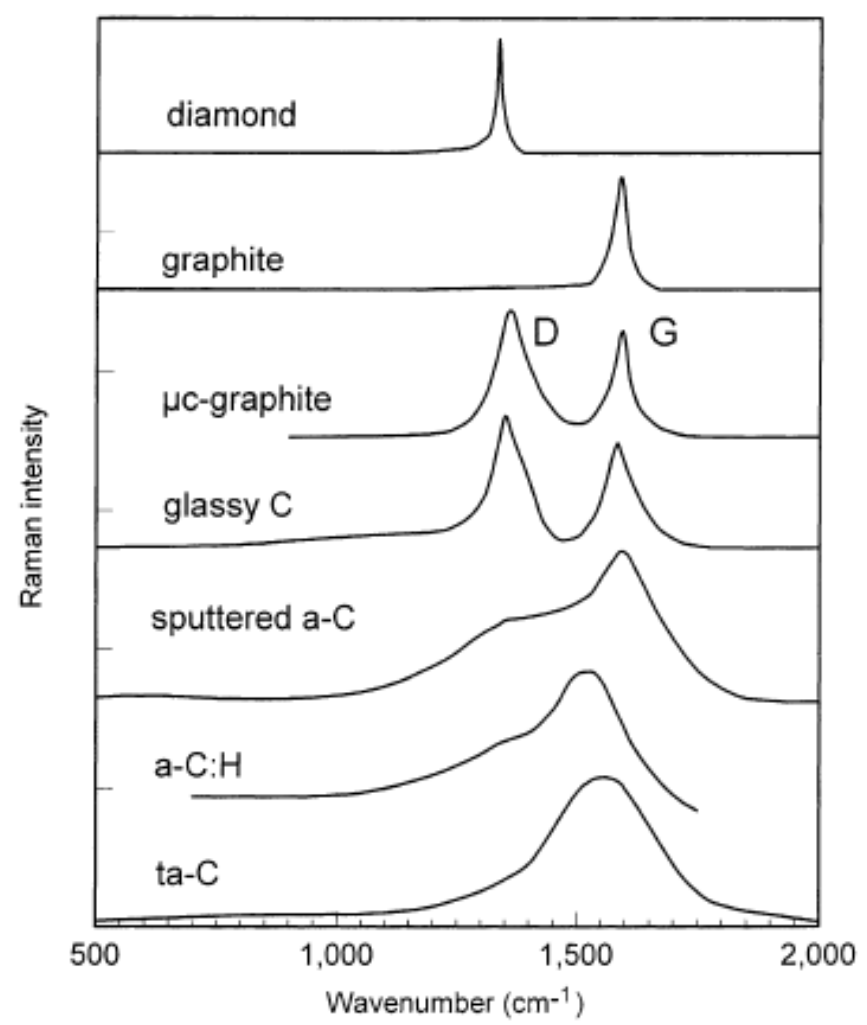

Figure 2.17. Raman Spectra of various carbon materials [16] 
Some factors such as $\mathrm{sp}^{2}$ clustering sites, the length of $\mathrm{C}-\mathrm{C}$ bonds and its angle, concentration of $\mathrm{sp}^{2}$ cluster or chain and $\mathrm{sp}^{3} / \mathrm{sp}^{2}$ ratio could affect the Raman spectra of amorphous carbons [73]. Figure 2.18 summarizes the effect of these factors on intensities and position of $D$ and $G$ peaks. As it can be deduced from the picture, due to complicated relationships among these factors, assessing $\mathrm{sp}^{3}$ content is tough. In addition, $\mathrm{sp}^{2}$ sites are dominant in visible excitation range as it is 50-250 times higher than $\mathrm{sp}^{3}$ sites. Moreover, investigation of $\mathrm{sp}^{3}$ content in DLC is very critical to understand film structure and characteristics as shown in different studies [73-75].

In order to obtain more information from DLC Raman spectra, Breit-Wigner-Fano ( BWF) is mostly used to fit G peak and Lorentzian is used to fit D peak [16]. In different amorphous carbon, the three-stage model is utilized to describe disorder carbon [76]. In stage 1, significant change from graphite structure to nano-crystalline graphite structure happens, that is accompanied by decrease in grain size while maintaining the aromatic ring. Thus, $G$ band moves toward higher wavenumbers up to $1600 \mathrm{~cm}^{-1}$ whereas $\mathrm{D}$ band is almost insignificant due to lack of disorder structure. Stage 2 is recognized when nano-crystalline graphite structure turns to a disordered $\mathrm{sp}^{2}$ rich amorphous carbon network, which leads to a loss of aromatic bonding and consequent shift of $\mathrm{G}$ peak to lower wavenumbers. In stage $3, \mathrm{sp}^{2}$ configuration rings transforms to short chains and $\mathrm{sp}^{3}$ content gradually increase toward $100 \%$. In this stage the ratio of $\mathrm{I}_{\mathrm{D}} / \mathrm{I}_{\mathrm{G}}$ is slowly reaching toward zero. Valuable information of DLC structure could be obtained by Raman spectrum [77]. For example, $\mathrm{sp}^{3}$ concentration in DLC could be obtained from studying the position, area, full with half maximum (FWHM), and the relative intensity $\left(I_{D} / I_{G}\right)$ of peaks. In this regard, de-convolution of $\mathrm{D}$ and $\mathrm{G}$ overlapped peaks could give more information about $\mathrm{D}$ and $\mathrm{G}$ peaks.

\subsubsection{Fourier transform infrared spectroscopy (FTIR)}

Changing in dipole moment during vibration means that the asymmetric molecule bond is $I R$ activated and consequently could give signal in the $I R$ band [78]. By utilizing the infrared band between 2.5 to $25 \mu \mathrm{m}$, the FTIR measurement could carry out in the range of $400-4000 \mathrm{~cm}^{-1}$. Advanced FTIR equipped with modern $I R$ techniques provide nondestructive, quick analysis of samples without laboratory preparations. Interaction between infrared radiation and sample is the basic of infrared spectroscopy whereby absorption infrared spectroscopy indicates that a photon is trapped inside the sample and emission infrared spectroscopy when a photon is radiated. 


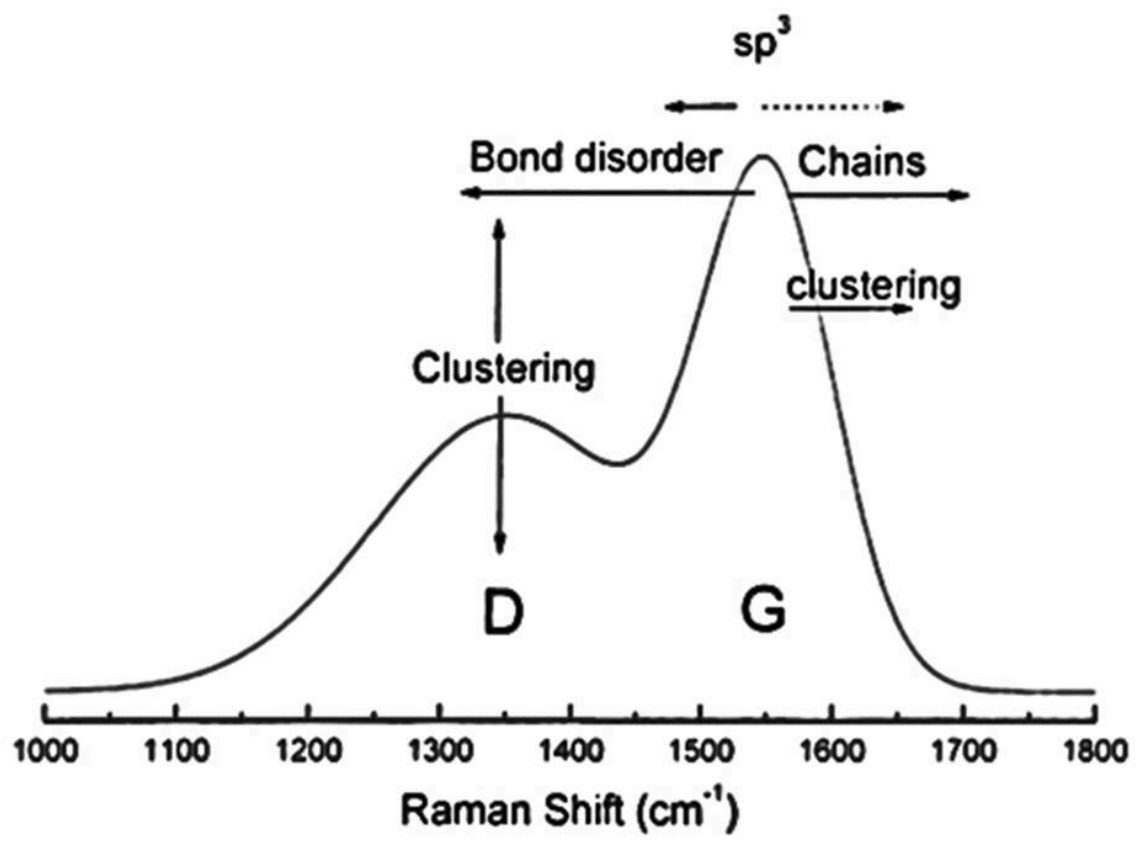

Figure 2.18. Schematic of an amorphous carbon (a-C) D and G peaks. Arrows show the factors affecting the height and position of Raman D and G peaks of amorphous carbons. Dotted arrow indicates the indirect influence of the sp3 content on increasing $\mathrm{G}$ position [16]

In FTIR, the photon would only just have sufficient energy to change the vibration or rotation state of the molecule and not enough energy to excite the electron. As it is illustrated in Figure 2.19, change in length and degree of bonds could describe molecule vibration. Therefore, atoms could have different vibration due to existence of various connections between molecules.
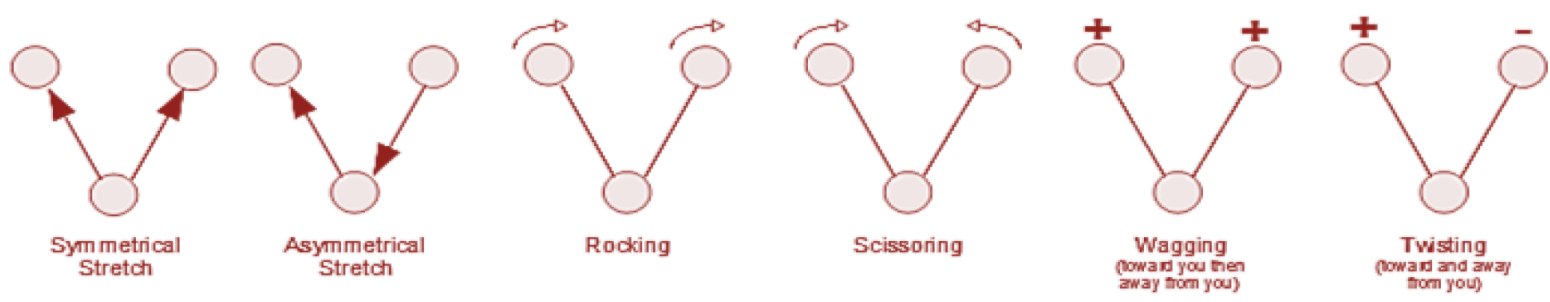

Figure 2.19. The illustration of some of the molecule vibrations [79].

In amorphous carbon studies, the FTIR technique could be very useful because of its high sensitivity to triple bonds. Furthermore, FTIR analysis is also useful for investigating the amount of hydrogen bonding in hydrogenated DLC. The infrared absorption band at $2900 \mathrm{~cm}^{-1}$ 
associated with the $\mathrm{C}-\mathrm{H}$ stretching vibration is used to estimate the $\mathrm{sp}^{3}$ ratio in $\mathrm{C}-\mathrm{H}$ bonds. In addition, the areas of absorption coefficients can be calculated for both $\mathrm{C}-\mathrm{H}$ bonds with $\mathrm{sp}^{3}$ carbon and $\mathrm{C}-\mathrm{H}$ bonds with $\mathrm{sp}^{2}$ carbon [80].

\subsubsection{X-ray photoelectron spectroscopy}

X-ray photoelectron spectroscopy (XPS) is a widely used technique to measure chemical analysis of materials. In addition, XPS gives quantitative information about elemental composition, chemical state and electronic state of the elements in the sample. XPS is based on the phenomenon called photoelectric effect. In this technique, soft X-ray photons with energy around 1-2 KeV are emitted to the surface of sample and knock out the core electrons or the valence electrons. Such electrons being knocked out or ejected, referred to as photoelectrons, are consequently detected by a hemispherical analyzer (HSA) and then analyzed by the detector. The detector measures the kinetic energy of the photoelectrons (equation 2.6), which is less than the absorbed X-ray photon energy, is equal to element binding energy.

$I P=E_{B}=h v-E_{k i n}-\Phi_{\text {work }}$

Where

$I P$ : ionization potential $(\mathrm{eV})$,

$E_{B}$ : binding energy $(\mathrm{eV})$,

$E_{k i n}$ : kinetic energy of the photoelectron $(\mathrm{eV})$,

$\Phi_{\text {work }}$ : work function of the sample (eV), and

$h v$ : photon energy $(\mathrm{eV})$

One of the characteristics of each element is having a unique set of binding energy, which is the basis of chemical analysis in XPS. In this technique, the calculated number of electrons ejected at a sequence of energies is plotted versus the binding energy. Therefore, the intensities of the peaks represent the elemental composition of the sample along with the peak shapes that give information about the chemical state for particular elements. Since XPS can only measured at 20 $\mathrm{nm}$ depth of the sample, it is known as a surface sensitive technique. XPS can be used to detect most of the elements except hydrogen and helium due to their low ionization potential.

Generally, several steps are needed to analyze the driven XPS data. In the first step, spectrum lines should be identified in order to find the particular elements associated with that energy 
level. In the second step, inelastic scattering background that comes from the emitted photoelectrons at different subsurface depth should be eliminated. Shirley and Tougaard [81] described the most commonly used methods to calculate inelastic background. In the third step, atomic sensitivity factors obtained from standard calibrations are used to calculate the actual XPS peak intensity for each element. By considering the fact that XPS peaks are proportional to the number of atoms in the analyzed volume, atomic concentration could be measured very straightforwardly.

\subsubsection{Near edge $X$-ray absorption fine structure spectroscopy}

NEXAFS gives information about local geometry and/or electronic structure of material in solid, gas or solution phases. Basically, NEXAFS is very close to X-ray absorption near edge structure (XANES) technique with a few differences. However, both techniques are considered as part of the X-ray absorption spectroscopy (XAS). Generally, NEXAFS refer to X-ray spectroscopy with lower energy level about 1-1000 eV while as XANES uses hard X-ray.

Measurement of photoelectron, fluorescence and Auger electron emission as a result of X-ray photoabsorption of atom core level is the fundamental of NEXAFS technique that is shown in

Figure 2.20. If a monochromic X-ray beam generated from synchrotron with sufficient energy to excite the core level electrons incident the surface of a material, three phenomenon can happen; emission of a photoelectron due to kicking out the electron to empty state, emission of auger electrons and fluorescence as a result of filling a inner-shell vacancy with electrons from other energy levels [82]. In order to measure the following emissions and gather XAS data, the X-ray beam photon energy should be tuned to an appropriate absorption edge. The term absorption edge in XAS study is related to excitation of core electron from specific atoms quantum numbers (n) of 1, 2 and 3, which corresponds to K-, L- and M-edges, respectively.

NEXAFS spectra could be performed in two modes: 1) Florescence Yield (FLY), 2) Total Electron Yield (TEY). In FLY mode, emitted florescence photons are measured, which is proportional to the number of X-ray photons absorbed by the samples [21]. In TEY mode, the total number of escaped electrons from the surface, including both photoelectrons and auger electrons, are counted and directly related to the X-ray absorption process. The FLY mode is relatively bulk sensitive and can reach about $100 \mathrm{~nm}$ depth for carbon $\mathrm{K}$ edge while TEY is more 
surface sensitive and is sensitive to only about few nanometer [83]. In some NEXAFS devices, instruments for both TEY and FLY are installed.

One of the most powerful techniques to study the DLC film electronic structure and bonding state in the atomic scale is NEXAFS. This technique can be used to determine $\mathrm{sp}^{2}$ and $\mathrm{p}^{3}$ concentration with fairly good accuracy. Thus, many studies conducted in this area use NEXAFS to analysis bonding configuration and concentration of $\mathrm{sp}^{2}$ and $\mathrm{sp}^{3}$ of nanodiamond and diamond like carbon coatings.

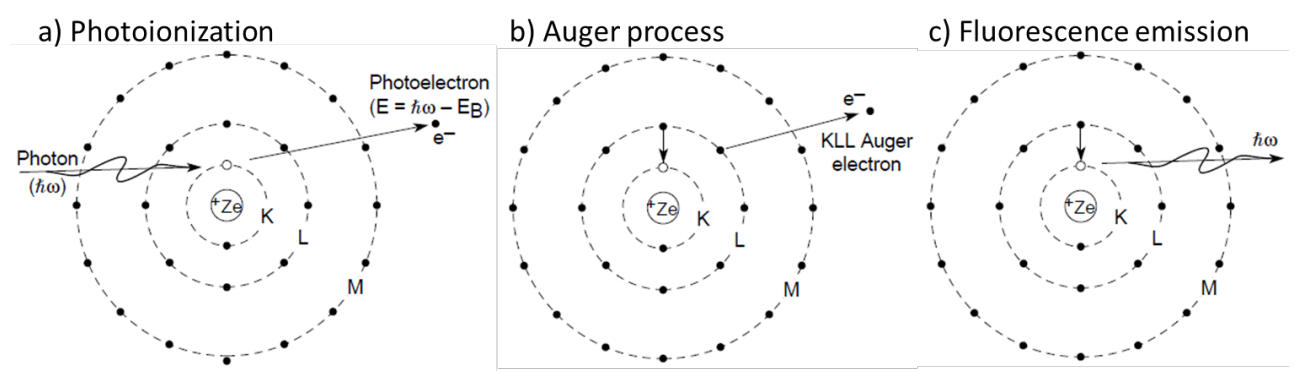

Figure 2.20. Schematic diagram of a) emission of photoelectron, b) auger, and c)

fluorescence [23]

\subsection{Property characterization of diamond-like thin films}

\subsubsection{Nanoindentation}

One of the major applied techniques to measure thin film mechanical properties is Nanoindentation. Precisely monitoring the penetration depth and displacement of the diamond nanoindentor tip when loaded into and then unloaded from the thin film is done with very small loads in the order of milli-Newtons or even micro-Newtons, and is the basis of nanoindentation. Hardness, elastic modulus, fracture toughness and yield strength of the thin film could be calculated by utilizing the measured data. The nanoindentor tip is one of the key components of this technique since different tips with various geometries could be utilized to such as Berkovich, cube corner, Vickers, and Knoop. The measured thin film hardness could easily be affected by factors such as thin film surface roughness and substrate hardness. The technique for calculating the hardness from the measurements is very critical as the calculated hardness value can differ significantly with different calculation techniques.

For hardness measurement of typical amorphous carbon, the force displacement curve is measured when the diamond tip penetrates into the surface. Figure 2.21 shows the loading and 
unloading curves of DLC film. For measuring the value of hardness, the tangent of the unloading curve should be measured and then extrapolating it to zero load. The hardness is calculated by using:

$H=0.0378 L_{\max } / h_{p}{ }^{2}$

Whereas the young modulus value is proportional to the slope of the tangent and can be found by the following equation;

$\left.E=0.179 \frac{\left(1-v^{2}\right)}{\left(h_{\max }-h_{p}\right) h_{p}}\right)$

where

$\mathrm{L}_{\max }$ : maximum load $(\mathrm{N})$,

v: Poisson ratio,

$\mathrm{h}_{\mathrm{p}}$ : plastic deformation

$h_{\max }-h_{p}$ : elastic deformation undergone by the film.

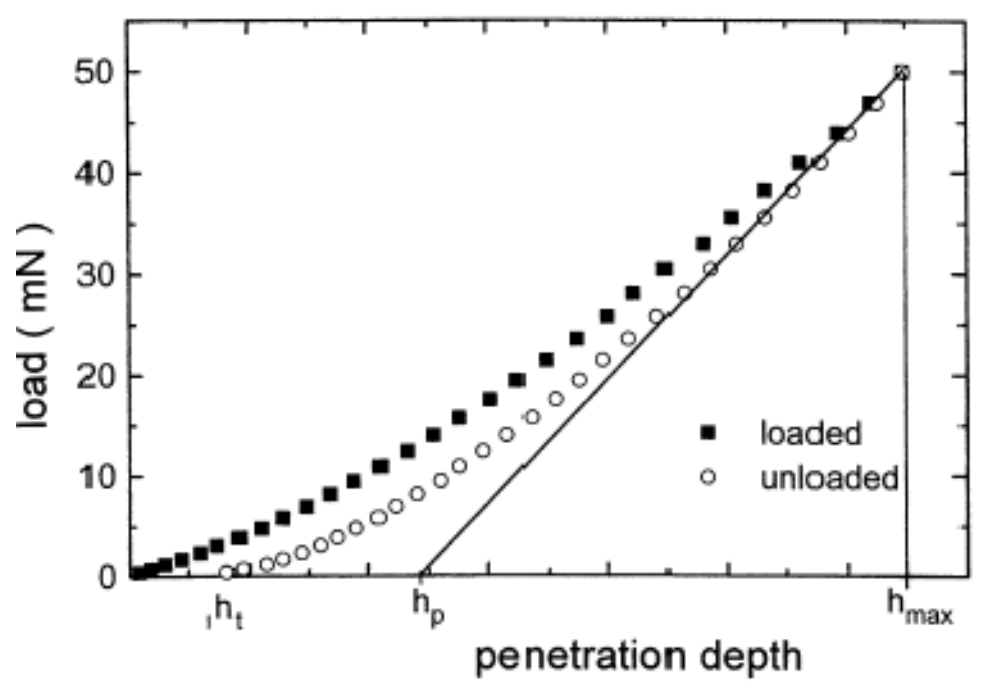

Figure 2.21. Loading -unloading curve from nano-indentation measurement [16]

\subsubsection{Friction and wear test}

When two contacting surfaces are in relative motion to each other, the dissipation of energy in between them is called friction, which manifests as a pair of opposing forces exerted to the two surfaces such that they oppose the relative motion. In fact, kinetic energy is transferred to heat due to friction, which can have consequences such as temperature-caused damages. In addition, 
another effect of friction is wear that can lead to damaging the surface and loss of performance. Friction force happens as a result of three major mechanisms including abrasion, shearing and adhesion as it is shown in Figure 2.22 [30]. Abrasion occurs when existing wear debris in the counterface scratches one of the surfaces. The shearing component of friction is the result of material being trapped in between two moving surfaces. The third component happens with the formation of adhesive junctions in between sliding surfaces.

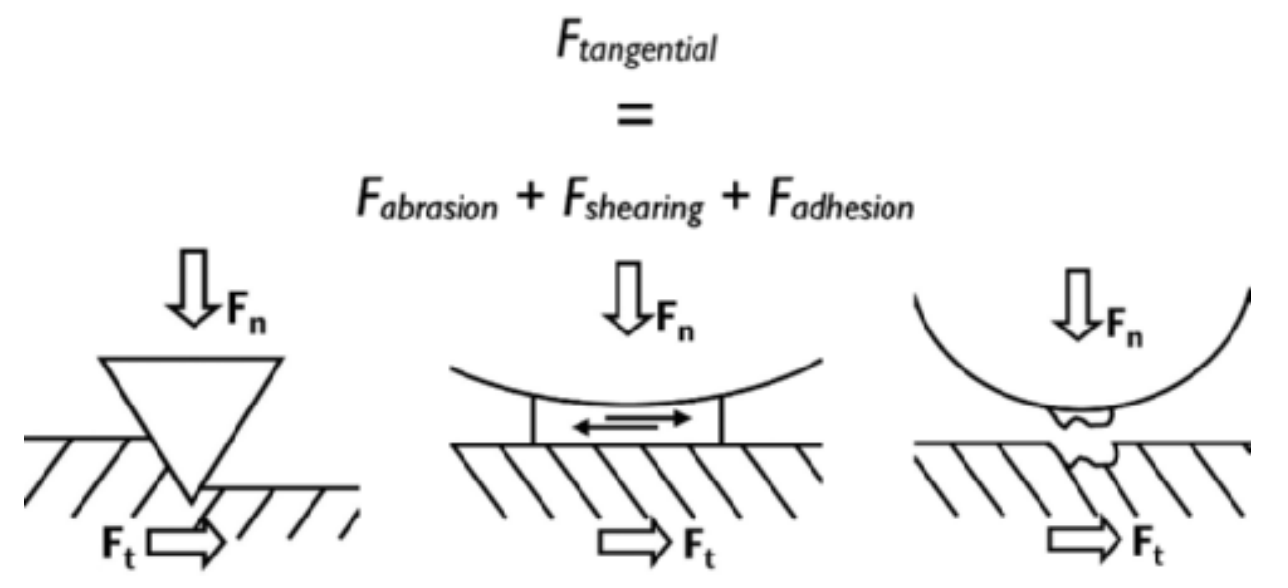

Figure 2.22. Schematic of three major components of friction [30]

In terms of DLC thin films, abrasive phenomenon would likely not occur because of their high hardness and high strain resistance. The contribution of shearing would not be significant because of high hardness of DLC as well. Though, shearing could be seen if the involvement of adhesive component was larger. Therefore, it can be concluded that the major fundamental phenomena in DLC friction is adhesion due to low surface roughness. Other properties of coatings like surface topography and mechanical properties can also play an important role in friction. Hence, surface chemistry controls the strength of adhesive junctions. Moreover, environmental conditions can strongly affect the friction. Friction coefficient values for different type of DLC are reported in the range of 0.001 to 0.7 [28].

Various Tribometers or friction testers such as ball-on-disc, ball-on-flat, pin-on-disc and flat-onflat, have been employed to measure the tribological performance of coatings. Therefore, some mathematical models have been developed based on coulomb approximation theory to measure coefficient of friction on coatings with tribometer. In this case, it is assumed that the surfaces are 
in close contact to each other and the friction force is proportional to the normal force. The schematic of ball-on-disc sliding test is illustrated in Figure 2.23.

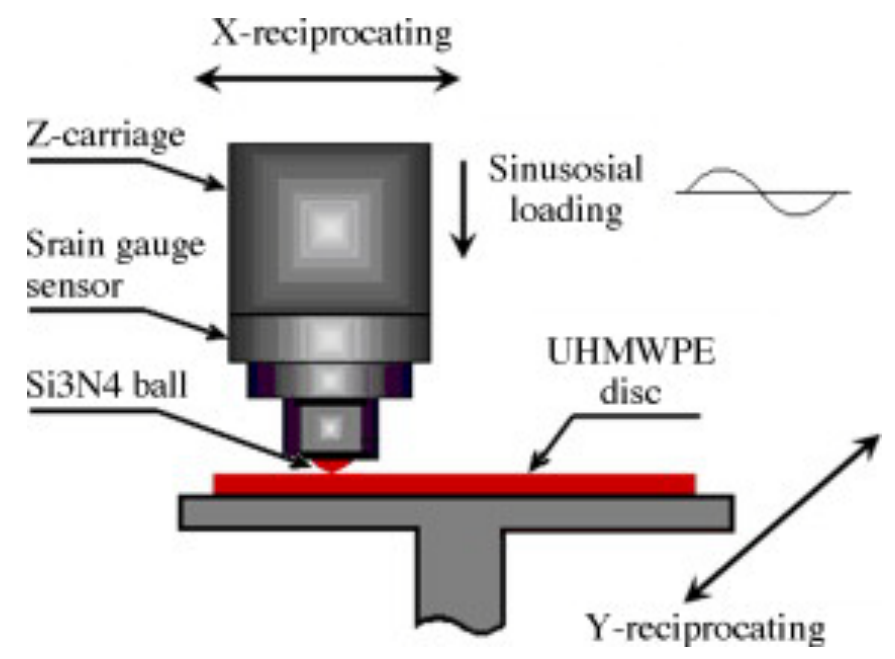

Figure 2.23. Schematic of ball-on-disc sliding test [84].

\subsubsection{Wettability and contact angle}

DLC is known for having small surface energy. In order to measure the surface energy, the contact angle $\theta\left(^{\circ}\right)$ of liquid on DLC surface could be measured. When a droplet is placed on the surface, surface energy would depend on three energies, the film surface energy $\gamma_{s v}(\mathrm{mN} / \mathrm{m})$, the liquid surface energy $\gamma_{l v}(\mathrm{mN} / \mathrm{m})$ and the interfacial energy of liquid and film $\gamma_{s l}(\mathrm{mN} / \mathrm{m})$, which is defined by Young's equation

$\gamma_{s v}=\gamma_{s l}+\gamma_{l v} \cos \theta$

Coating characteristic such as absorption, wetting and adhesion properties can be understood by surface energy that are also measured by contact angle. As is shown in Figure 2.24, a small contact angle is observed if the liquid spreads on the surface, and a large contact angle is achieved when the droplet beads on the surface. More specifically, if the contact angle was less than $90^{\circ}$ then wetting of the surface is favorable (hydrophilic). Therefore, larger angles (more than $90^{\circ}$ ) means wetting of the surface is unfavorable (hydrophobic). A high contact angle is the result of low surface energy. 


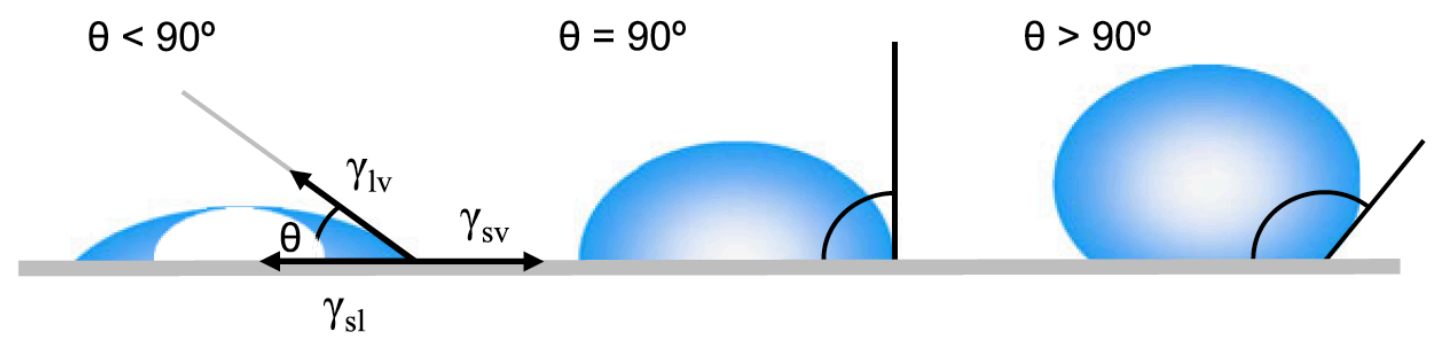

Figure 2.24. Schematic of different contact angles

Generally, the Young - Dupre equation is used to measure surface energy while the liquid surface energy $\gamma_{l v}$ and the interfacial energy of liquid are equal.

$E=\gamma(1+\cos \theta)$

Where $\gamma$ is the water surface tension at $20^{\circ} \mathrm{C}$ which is equal to $72.8 \mathrm{mN} / \mathrm{m}$ and $\theta$ is the contact angle $\left({ }^{\circ}\right)$.

\subsection{Atomic force microscopy (AFM)}

Atomic Force Microscopy (AFM) is a powerful technique to collect high-resolution images in three dimensions from surface topography of either insulator or conductive materials. The interaction between the sample surface and the silicon made sharp tip that is placed on a flexible cantilever, is monitored by reflecting a laser beam from the back of cantilever and collecting the reflected beam by a photodiode detector. Two of the most common AFM modes are contact and tapping modes. There is a gentle physical contact between the sample and the tip in contact mode. Whilst the basis of tapping mode is the small piezoelectric element mounted on the AFM tip sensing the ups and downs of the sample surface without touching. In contact mode, change in the sample topography is monitored by the deflection in the cantilever while scanning the surface by constant force, in which the scanning motion is for generating an image. The tapping mode uses high-resolution technique to collect images from small single molecules. AFM could work in ambient air and provide three-dimensional images with higher resolutions than other techniques like SEM. However, AFM can only scan small area in the realm of $150 \times 150 \mu \mathrm{m}$ with a maximum height around a few micrometers. AFM could be used to study the topography of DLC and other surfaces like plasma surface treated polymers. 


\section{CHAPTER 3 \\ MATERIALS AND METHODS}

\subsection{Ion Beam deposition system}

A custom designed ion beam system manufactured by 4 Wave Inc. located at the Department of Mechanical Engineering in the University of Saskatchewan was used to synthesize the thin films on PET and silicon substrates. A picture of the IBD system is presented in appendix, Figure A.1. The system consists of a high vacuum chamber, a pumping system (comprise of mechanical and turbo pumps), a substrate holder (that can be inclined from $0^{\circ}$ to $90^{\circ}$, three targets and two EndHall low energy ion source. One source is used for direct ion beam deposition and the other is used for sputtering as it is illustrated in Figure 3.1. The distance between the substrate holder, which is tilted $45^{\circ}$, and the E-H ion beam etch source is about $300 \mathrm{~mm}$. Ion beam etching, ion beam deposition and ion beam sputtering could be performed in this versatile system.

By utilizing the end-hall ion source I, ion beam deposition, ion beam assisted deposition, etching and pre-cleaning the surface could be done, while the end-hall ion source $\Pi$ could be used for sputtering. Hydrogenated DLC could be synthesized from hydrocarbon gases like methane and acetylene using the E-H ion source I. Sputtering of graphite particles using the E-H ion source $\Pi$ is the basis of deposition of hydrogen free DLC. The simultaneous use of both end halls also makes it possible for deposition of metal or non-metal doped DLC.

In the present thesis work, doped DLC and pure DLC samples were synthesized on PET and silicon using the E-H ion source manufacture by Kaufman \& Robinson, Inc. High ion current (up to 5 Amps) and low energy ion beam (35-210 v), uniform deposition in large scale, as well as ease of operation and maintenance are the biggest advantage of griddles E-H ion source. Polyethylene terephthalate (PET) polymer purchased from Goodfellow Co. with the thickness of $100 \mu \mathrm{m}$ were used in this study. Samples were carefully cleaned with an alcohol bath, washed with distilled water, and then dried at room temperature. A piece of cleaned silicon wafer with 
the size of $1 \mathrm{~cm} \times 1 \mathrm{~cm}$ as a reference sample was installed into the system along with the polymer substrates.

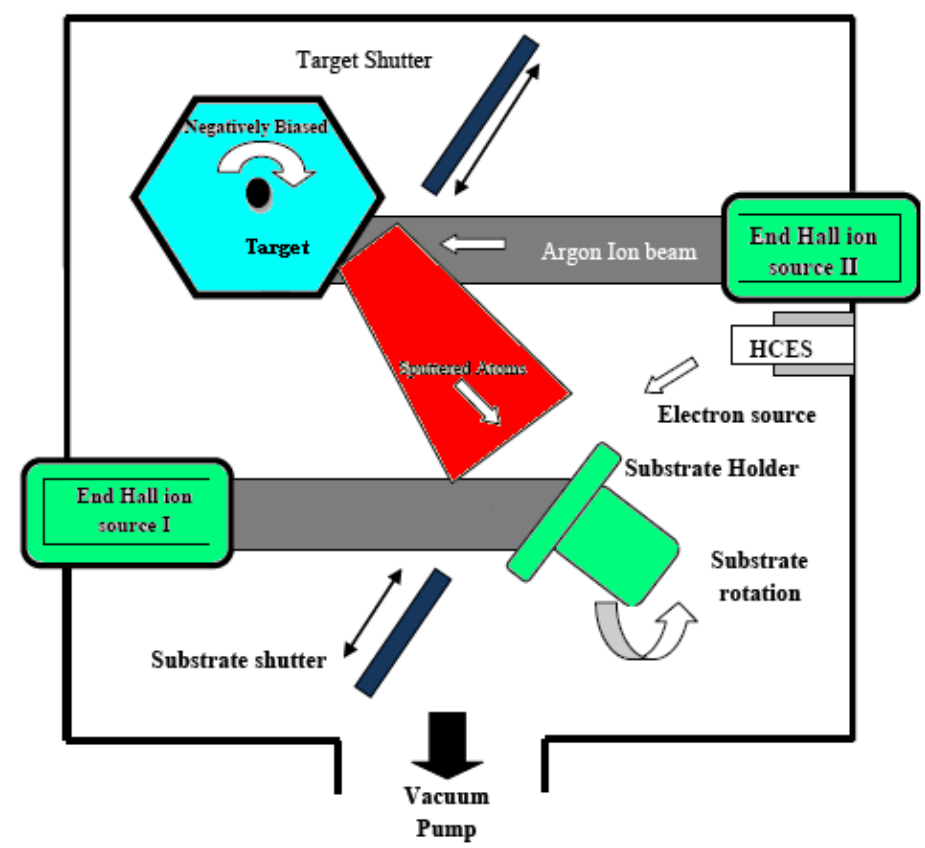

Figure 3.1. A schematic of the IBD system configuration [85]

Table 3.1. Deposition parameters of doped DLC samples

\begin{tabular}{|c|c|c|c|c|c|c|}
\hline Parameters & DLC-M & NDLC-1 & NDLC-2 & NDLC-3 & $\begin{array}{l}\text { SDLC- } \\
400\end{array}$ & $\begin{array}{l}\text { SDLC- } \\
800\end{array}$ \\
\hline $\begin{array}{c}\text { Ion Current } \\
\text { (A) }\end{array}$ & 3 & 3 & 3 & 3 & 3 & 3 \\
\hline $\begin{array}{c}\text { Total gas } \\
(\mathrm{SCCM}) \\
\left(\mathrm{Ar}+\mathrm{CH}_{4}+\mathrm{N}_{2}\right)\end{array}$ & 20 & 21 & 22 & 23 & 20 & 20 \\
\hline Argon & 12 & 12 & 12 & 12 & 12 & 12 \\
\hline Methane & 8 & 8 & 8 & 8 & 8 & 8 \\
\hline Nitrogen & - & 1 & 2 & 3 & - & - \\
\hline $\begin{array}{l}\text { Target bias } \\
\text { (v) }\end{array}$ & - & - & - & - & 400 & 800 \\
\hline $\begin{array}{l}\text { Deposition } \\
\text { time (h) }\end{array}$ & 4 & 4 & 4 & 4 & 4 & 4 \\
\hline $\begin{array}{c}\text { Deposition } \\
\text { temperature } \\
{ }^{\circ} \mathrm{C}\end{array}$ & 25 & 25 & 25 & 25 & 25 & 25 \\
\hline $\begin{array}{c}\text { Coating } \\
\text { thickness } \\
(\mathbf{n m})\end{array}$ & 450 & 320 & 290 & 240 & 460 & 510 \\
\hline
\end{tabular}


Based on the previous research conducted in our group, direct ion beam deposition with a mixture of $\mathrm{Ar} / \mathrm{CH}_{4}(12 / 8) \mathrm{SCCM}$ was used to deposit hydrogenated DLC. Mixtures of argon (Ar), methane $\left(\mathrm{CH}_{4}\right)$ and nitrogen $\left(\mathrm{N}_{2}\right)$ were used as precursor gases for N-DLC thin films. The mean ion energy of depositions was kept in between $55 \mathrm{eV}$ and $65 \mathrm{eV}$ and the current was $3 \mathrm{~A}$ for all the depositions. In all cases, depositions were performed in room temperature for a period of $4 \mathrm{~h}$ while the substrate holder was inclined at an angle of $45^{\circ}$ to the incident beam. Ion beam base pressure was $3 \times 10^{-7}$ Torr and working pressure was kept around $5 \times 10^{-4}$ Torr. Different concentrations of nitrogen doped DLC were deposited under three different nitrogen flow rates (1 SCCM, 2 SCCM and 3 SCCM). Furthermore, Si doped hydrogenated DLC was achieved by simultaneously running ion beam sputtering of silicon targets with different target bias (400 and 800 volts) and low energy etching source with methane precursor. Deposition parameters of NDLC and Si-DLC are summarized in Table 3.1.

Plasma surface treatments were also performed using the E-H ion source I in three different environments. Oxygen/ argon, nitrogen/ argon and argon/ argon environments were used to etch the PET surface before DLC deposition for $30 \mathrm{~min}$ and 60 minutes. The total flow of the gas mixture was kept at 20 SCCM while the argon background flow was kept at 12 SCCM in all the samples. In the next step, DLC depositions with two different hydrocarbon precursors were performed to synthesize pure DLC on plasma surface treated samples. Table 3.2 shows the deposition parameters for DLC samples after surface treatment.

Table 3.2. Deposition parameters of pure DLC with two different hydrocarbon gas

\begin{tabular}{ccc}
\hline Parameters & DLC-M & DLC-A \\
\hline Ion Current (A) & 3 & 3 \\
Total gas $(\mathbf{s c c m})$ & 20 & 18 \\
$\left(\mathbf{A r}+\mathbf{C H}_{\mathbf{4}} / \mathbf{C}_{\mathbf{2}} \mathbf{H}_{2}\right)$ & 12 & 12 \\
Argon & 8 & - \\
Methane & - & 4 \\
Acetylene & 4 & 4 \\
Deposition time (h) & 25 & 25 \\
Deposition temperature ${ }^{\circ} \mathbf{C}$ & 450 & 435 \\
Coating thickness $(\mathbf{n m})^{\circ}$ & & \\
\hline
\end{tabular}




\subsection{Structural characterization}

Microstructure characterization by Raman spectroscopy was carried out using a Renishaw Invia Reflex Raman microscope (Renishaw, Gloucestershire, UK), located at Saskatchewan Structural Science Center (SSSC), University of Saskatchewan, operated under a $514 \mathrm{~nm}$ Ar ion laser with a spot size of approximately $2 \mu \mathrm{m}$. During operation, three different spots were selected for testing with $30 \mathrm{sec}$ exposure times for better Raman results. A picture of the Raman spectroscope is presented in appendix as Figure A.2. Fourier Transform Infrared (FTIR) spectroscopy located at Saskatchewan Structural Science Center (SSSC), University of Saskatchewan, using Illuminat IR II FTIR microscope accessory (Smith's Detection, Danbury, CT) equipped with liquid nitrogen cooled MCT detector. ATR-FTIR measurements were performed in the range of 4000$650 \mathrm{~cm}^{-1}$.

XPS measurements were performed by the Omicron Multiprobe system of the REIXS surface science facility at the Canadian Light Source (CLS), University of Saskatchewan, using a monochromatized A1 K (alpha) x-ray source and a Sphera EA 125 hemispherical electron energy analyzer with the kinetic energies ranging from 600 to $1500 \mathrm{eV}$. An electron gun was also used to neutralize the charging effect on the polymer surface. Film composition and chemical structure was analyzed with CasaXPS software. The bonding nature of carbon in DLC and NDLC samples deposited on silicon were studied by Near Edge X-ray Absorption Spectroscopy (NEXAS) carried out at SGM beamline at the Canadian Light Source, University of Saskatchewan. Two modes of total electron yield mode (TEY) and fluorescence yield mode (FLY) were used to study C K-edge of N-DLC samples. NEXAFS energy window ranged from $280 \mathrm{eV}$ to $320 \mathrm{eV}$.

\subsection{Surface property characterization}

AFM measurements were performed in tapping mode in air by a Dimension Hybrid Nanoscope system (Veeco Metrology Group) located at the Department of Chemistry, University of Saskatchewan, using commercial AFM probes (Veeco Metrology Group). The AFM was mounted with an optical microscope (TE2000, Nikon) as presented in appendix in Figure A.3. The images of samples were obtained in air without any surface damage.

New View 8000 optical profilometer manufactured by Zygo located at Department of Mechanical Engineering, University of Saskatchewan, was also utilized to investigate thickness 
of coating and sample surface topography before and after deposition. A picture of the optical profiler system is displayed in appendix in Figure A.4.

The water contact angle of PET substrate with and without DLC coatings were calculated with a goniometer Located at the Department of Soil Science, University of Saskatchewan, equipped with micropipette, which gives precise control of droplet size and injection force, Nikon Cool Pix 8400 camera with $3264 \times 2448$ resolutions, a sample stage and contact angle analysis software. The schematic of the goniometer is shown in Figure 3.2. The measurement was carried out in three points with liquid distilled water at room temperature. The obtained images were analyzed with open source multi-platform java image processing program ImageJ, using the Low Bond Axisymmetric Drop Shape Analysis Model of Drop Shape Analysis (LB_ADSA) plug in. Young - Dupre equation $[E=\gamma(1+\cos \theta)]$ was used to measure surface energy [86]. Where $\gamma$ is water surface tension at $20^{\circ} \mathrm{C}$, which is equal to $72.8 \mathrm{mN} / \mathrm{m}$, and $\theta$ is the contact angle $\left(^{\circ}\right)$.

\subsection{Mechanical property characterization}

Hardness and Young's modules of DLC films on Si substrates were measured by nanoindentation using a Universal Materials Tester (UMT) manufactured by the Center for Tribology (CETR) Inc. located at the Department of Mechanical Engineering, University of Saskatchewan. The indentation testing was performed on nine different locations for each sample with the use of a Berkovich indenter. The mechanical properties of DLC films were calculated by loaddisplacement curves based on Oliver and Pharr method [87]. A picture of the UMT is displayed in appendix as Figure A.5.

Another UMT purchased from CETR Inc., located at the Department of Mechanical Engineering, University of Saskatchewan, was used to perform the scratch test to measure the adhesion of the thin films. The scratch test was performed in a fully computerized UMT with a Tungsten carbide blade as the scratching object. A load was applied via a closed-loop servomechanism and was programmed to increase linearly from $0.1 \mathrm{mN}$ to $0.5 \mathrm{mN}$. The final scratch line was observed under optical microscope in three different locations (beginning, middle and the end). Ball-on-disc tribometer was used in the same system to measure the coefficient of friction (COF) of the film with $4 \mathrm{~mm}$ ultra high molecular weight polyethylene (UHMWPE) balls. The tests were performed under 14400 reciprocating cycles with $5 \mathrm{~mm}$ stroke operating 
under $1 \mathrm{~N}$ loading force in an ambient atmosphere $\left(22^{\circ} \mathrm{C}\right.$ and a relative humidity of $\left.40 \%\right)$. The picture of the tribometer is shown in appendix, Figure A.6.

Micropipette:

Manipulation of different

volumes and types of solutions

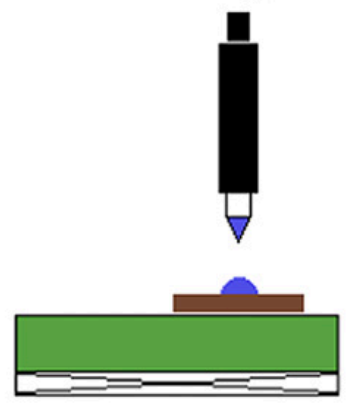

Stage

-Control parameters (Temp,

Humidity, Wind Speed and Angle)

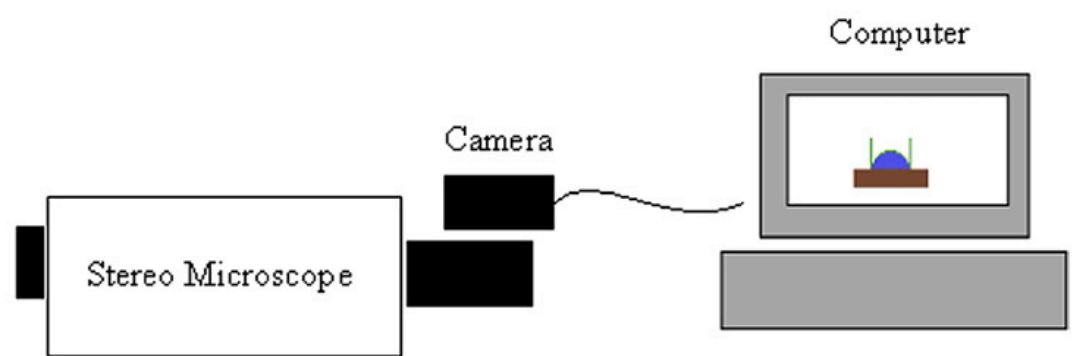

Viewing and image acquisition at

Image digitization to accurately different resolutions

determine contact angles

-Different types and sizes of

samples

Figure 3.2. Schematic of image acquisition and contact angle measurement 


\section{CHAPTER 4}

\section{RESULTS AND DISSCUSSIONS}

\subsection{Ion treatment of PET substrate films}

\subsubsection{X-ray photoelectron spectroscopy (XPS)}

Figure 4.1.a illustrates the XPS spectra of $\mathrm{C} 1 \mathrm{~s}$ and O1s in bare (untreated) PET. Four major peaks at 284.6, 286.3, 288.6 and $291.4 \mathrm{eV}$ are identified after peak de-convolution for $\mathrm{C} 1 \mathrm{~s}$ spectrum in Fig. 4.1a. The peak at $284.6 \mathrm{eV}$ can be assigned to $\mathrm{C}-\mathrm{C}$ or $\mathrm{C}-\mathrm{H}$ bonds, non-oxidized carbons in aromatic ring. Located at 286.3 is the peak associated to ethylene carbons that are singly bounded with oxygen (C-O) while the peak of double bonded carboxylate carbons (ester $\mathrm{O}=\mathrm{C}-\mathrm{O}$ ) is located at 288.6. The small satellite peak $\mathrm{d}$ at 291.4 is because of the aromaticity within the PET molecule [88]. For O1s XPS spectrum in Figure 4.1.b, two major peaks at 531.7 and $533.3 \mathrm{eV}$ are attributed to $\mathrm{O}=\mathrm{C}$ and $\mathrm{O}-\mathrm{C}$, respectively.
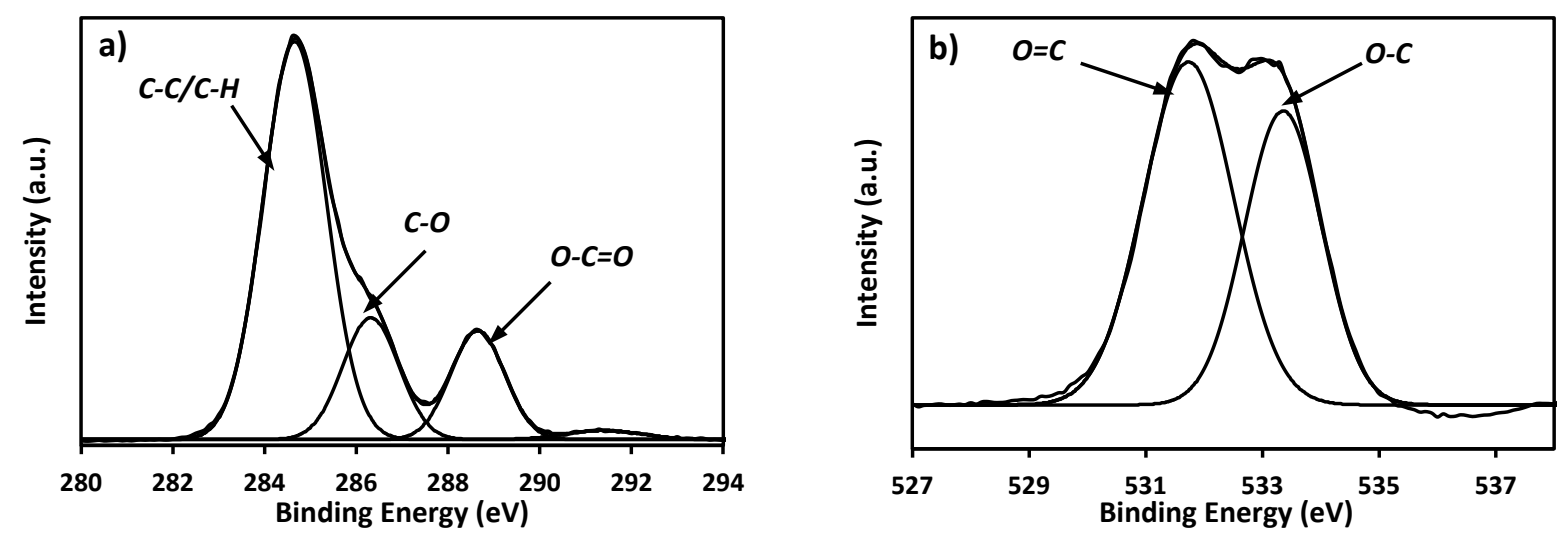

Figure 4.1. De-convolution of XPS spectra of bare PET: a) C1s and b) O1s

$\mathrm{Ar} / \mathrm{Ar}, \mathrm{Ar} / \mathrm{O}_{2}$ and $\mathrm{Ar} / \mathrm{N}_{2}$ ion treatments were performed on PET films for 30 and 60 minutes. Table 4.1 summarizes the bonding states and $\mathrm{O} / \mathrm{C}$ ratio of the polymer after different ion treatments calculated based on XPS spectra. The results show that $\mathrm{O} / \mathrm{C}$ ratio decreases after 
treatments except 30 minutes $\mathrm{Ar} / \mathrm{O}_{2}$ ion treatment. Increasing in $\mathrm{O} / \mathrm{C}$ ratio implies the formation of oxygen functional groups on the PET film surface after exposure to $\mathrm{Ar} / \mathrm{O}_{2}$ ion bombardment for 30 mins whereas decreasing in $\mathrm{O} / \mathrm{C}$ ratio indicates breaking of $\mathrm{C}-\mathrm{O}$ and $\mathrm{O}=\mathrm{C}-\mathrm{O}$ bonds and formation of free radicals on the surface. C1s XPS spectra of PET films after 30 and 60 minutes $\mathrm{Ar} / \mathrm{O}_{2}$ ion treatment, simplified as oxygen treatment, are illustrated in Figure 4.2.

Table 4.1. Bonding states of ion treated PET films

\begin{tabular}{|c|c|c|c|c|c|c|c|}
\hline & $O / C$ & $C=C \%$ & $\mathrm{C}-\mathrm{O} \%$ & $O-C=O \%$ & $C-\mathrm{N} \%$ & $C=O \%$ & $O-C=O-H$ \\
\hline Bare PET & 0.37 & 65.2 & 17.8 & 15.2 & & & \\
\hline $30-\mathrm{Ar} / \mathrm{O}_{2}$ & 0.44 & 64.5 & 14.5 & 12.9 & & 5.9 & 2.2 \\
\hline $60-\mathrm{Ar} / \mathrm{O}_{2}$ & 0.25 & 75.7 & 15.1 & 9.2 & & & \\
\hline $30-A r / A r$ & 0.31 & 78.3 & 12.3 & 9.5 & & & \\
\hline $60-A r / A r$ & 0.24 & 85.7 & 10.3 & 4.0 & & & \\
\hline $30-\mathrm{Ar} / \mathrm{N}_{2}$ & 0.25 & 68.3 & 18.5 & 7.0 & 6.1 & & \\
\hline $60-A r / N_{2}$ & 0.23 & 67.9 & 15.0 & 10.0 & 7.2 & & \\
\hline
\end{tabular}

New oxygen functional groups have been formed on PET films after $30 \mathrm{~min} \mathrm{Ar} / \mathrm{O}_{2}$ ion treatment with peaks at $286.2 \mathrm{eV}$ and $288.6 \mathrm{eV}$, attributed to $\mathrm{C}=\mathrm{O}$ and $\mathrm{O}-\mathrm{C}=\mathrm{O}-\mathrm{H}$ bonds, correspondingly [89]. However, in $60 \mathrm{~min} \mathrm{Ar} / \mathrm{O}_{2}$ ion treated films, the fraction of $\mathrm{C}=\mathrm{C}$ bond increases with the reduction of $\mathrm{C}-\mathrm{O}$ and $\mathrm{O}-\mathrm{C}=\mathrm{O}$ bonds, that could result in the formation of free bonds on the PET surface. Generally, polymer surface modification happens due to three different processes defined as replacing, etching, and polymerization. The initial stage is replacing and etching follows. In the case of $\mathrm{Ar} / \mathrm{O}_{2}$ ion treatment, new functional groups forms during the initial stage by replacing the polymer bonds, while etching is more likely to dominant after the initial replacing [90]. 

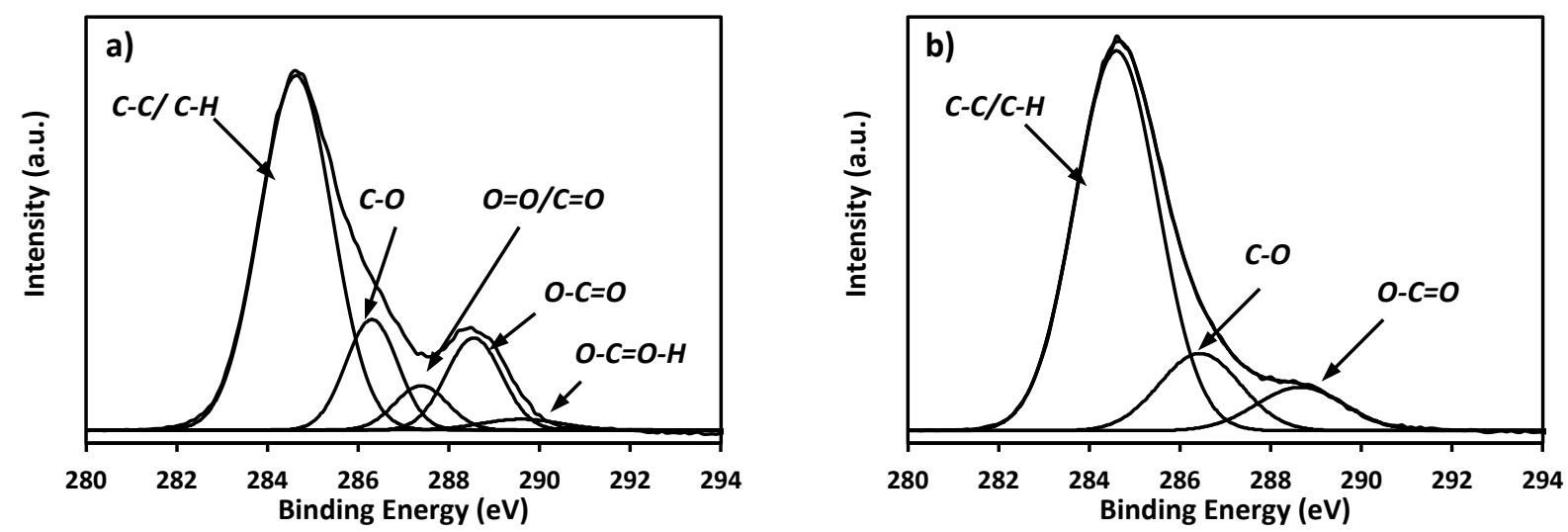

Figure 4.2. C1s XPS spectra of $\mathrm{Ar} / \mathrm{O}_{2}$ ion treated PET films a) for $\mathrm{r} 30 \mathrm{~min}$ and b) from $60 \mathrm{~min}$

O1s XPS spectra of $\mathrm{Ar} / \mathrm{O}_{2}$ ion treated samples are displayed in Figure 4.3. No shoulder was detected for $\mathrm{Ar} / \mathrm{O}_{2}$ treated samples. Table 4.2 presents the bonding states of oxygen of samples with different treating time. The results show that the percentage of $\mathrm{O}=\mathrm{C}$ bonds increases and $\mathrm{O}-$ $\mathrm{C}$ bond decreases comparing to bare PET before treatment. Higher oxygen content after 30 minutes treatment could be due to formation of new carbonyl bonds on the surface while by increasing treatment time, repetitive oxygen ion bombardment on the surface could break polymer bonds on the surface and form free radicals [91], thus decreasing $\mathrm{O}$ content.
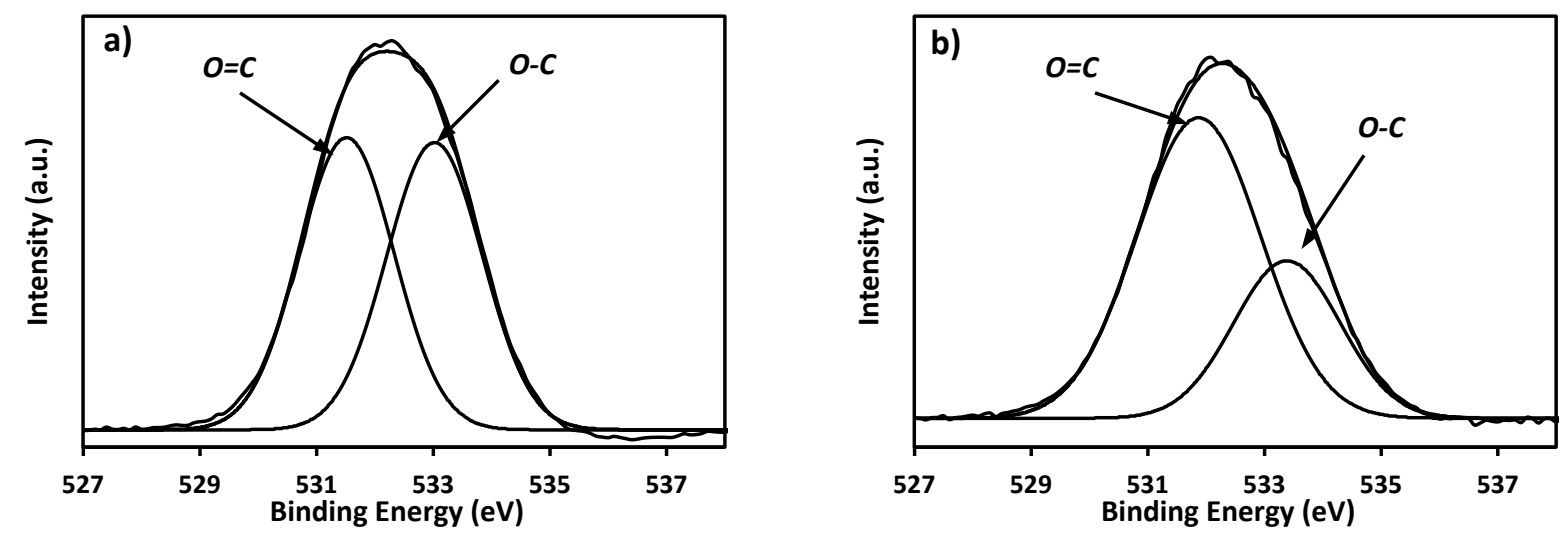

Figure 4.3. O1s XPS spectra of Oxygen treated PET film a) after $30 \mathrm{~min} \mathrm{~b}$ ) after $60 \mathrm{~min}$ 
Table 4.2. O1s Bonding state of plasma treated PET films

\begin{tabular}{lcc}
\hline & $\boldsymbol{O}=\boldsymbol{C} \%$ & $\boldsymbol{O}-\boldsymbol{C}$ \\
\hline Bare $\boldsymbol{P E T}$ & 57.7 & 42.3 \\
$\mathbf{3 0}-\boldsymbol{A r} / \boldsymbol{O}_{2}$ & 73.6 & 26.4 \\
$\mathbf{6 0}-\boldsymbol{A r} / \boldsymbol{O}_{2}$ & 69.0 & 31.1 \\
$\mathbf{3 0}-\boldsymbol{A r} / \boldsymbol{A r}$ & 86.1 & 13.9 \\
$\mathbf{6 0}-\boldsymbol{A r} / \boldsymbol{A r}$ & 84.5 & 15.5 \\
$\mathbf{3 0}-\boldsymbol{A r} / \boldsymbol{N}_{2}$ & 83.2 & 16.8 \\
$\mathbf{6 0}-\boldsymbol{A r} / \mathbf{N}_{2}$ & 69.5 & 30.5 \\
\hline
\end{tabular}

PET films were also treated by $\mathrm{Ar} / \mathrm{N}_{2}$ ions for 30 and 60 minutes and their $\mathrm{C} 1 \mathrm{~s}$ XPS spectra are shown in Figure 4.4. Formations of new $\mathrm{C}-\mathrm{N}$ bonds have been observed under both treatment times [92]. The lowest $\mathrm{O} / \mathrm{C}$ ratio is also found after $\mathrm{N}$-treatment that could be due to the high etching effect of nitrogen ions. Therefore, $\mathrm{O}-\mathrm{C}$ and $\mathrm{O}=\mathrm{C}$ bonds are broken, which results in the formation of new bonds between nitrogen and carbon. nitrogen ions have an average energy of approximately $60 \mathrm{eV}$, enough to break the carbon-oxygen bonds and form new bonds with carbon. This effect could rise by increasing the treatment time as $\mathrm{C}-\mathrm{N}$ percentage is higher for longer treatment time presented in Table 4.1.
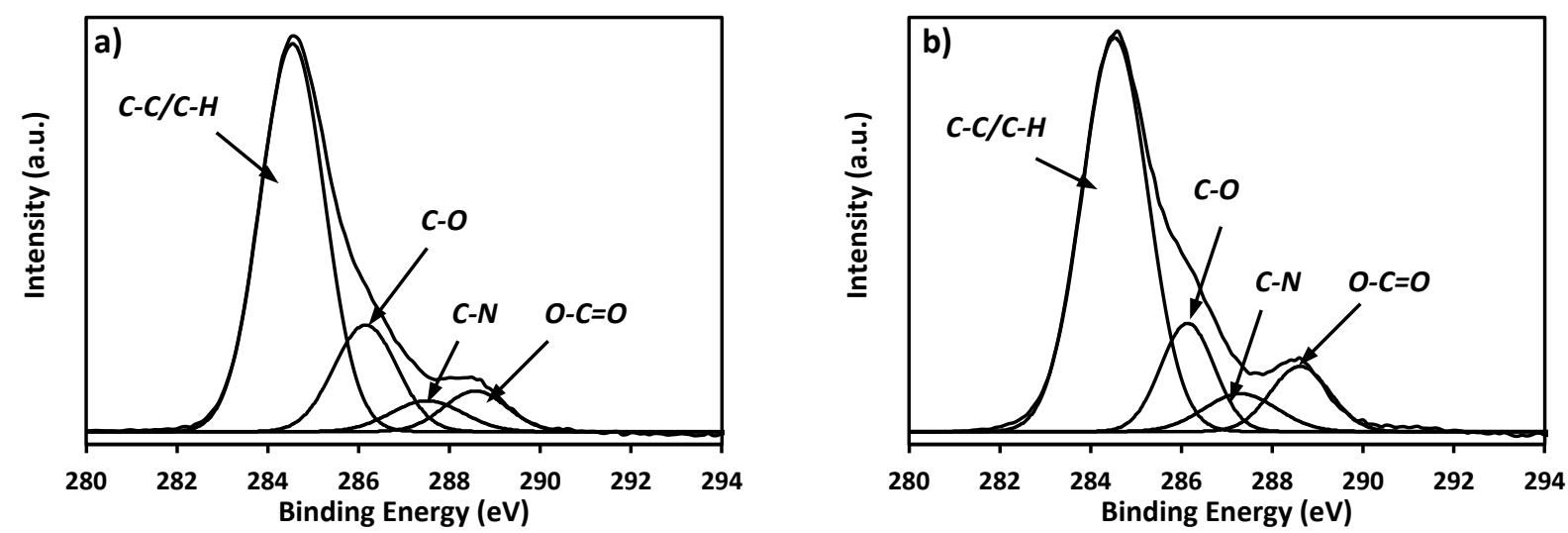

Figure 4.4. C1s XPS spectra of Argon treated PET film a) for $30 \mathrm{~min}$ and b) for $60 \mathrm{~min}$

In addition to oxygen and nitrogen ion treatment, PET films were subjected to pure argon ion treatment. Although the radius of argon ions is bigger than that of oxygen and nitrogen ions, argon ions have enough energy to break $\mathrm{O}-\mathrm{C}=\mathrm{O}$ and $\mathrm{O}-\mathrm{C}$ bonds on the surface as $\mathrm{O}-\mathrm{C}=\mathrm{O}$ bonds on the surface after 60 minutes argon treatment have been found to be with the lowest fraction 
(Table 4.1). Furthermore, by increasing the treatment time chemical state of PET has been changed as it can be seen in Figure 4.5.
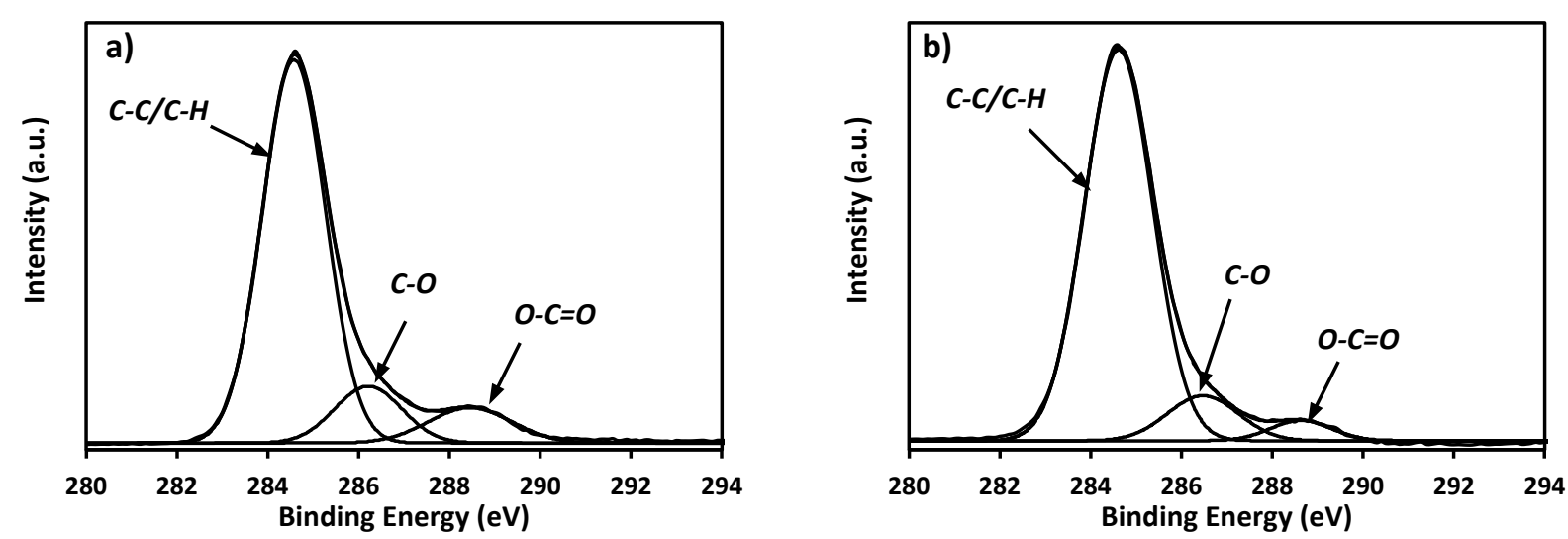

Figure 4.5. C1s XPS spectra of Argon treated PET film a) for $30 \mathrm{~min}$ and b) for $60 \mathrm{~min}$

\subsubsection{Fourier transform infrared spectroscopy (FTIR)}

FTIR study of PET films before and after 60 minutes $\mathrm{Ar} / \mathrm{O}_{2}$ treatment is shown in Figure 4.6. As it can be seen, the intensity ratio of carbonyl $\mathrm{C}=\mathrm{O}$ to asymmetric $\mathrm{C}-\mathrm{C}-\mathrm{O}$ bonds decreases after ion treatment. It further demonstrates the effect of ion treatment on polymer bonding structure, confirming the XPS results described in section 4.1.1.

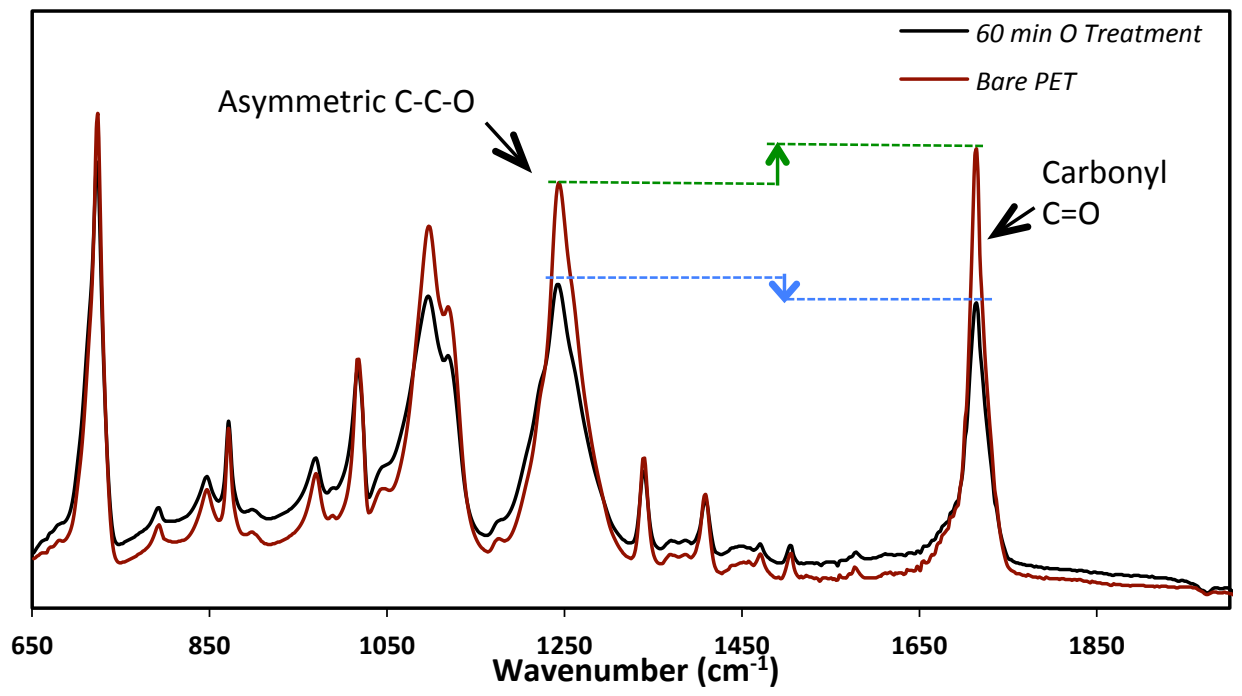

Figure 4.6. ATR-FTIR Spectra of bare PET and 60 min Oxygen treated PET film 


\subsubsection{Atomic force microscopy analysis (AFM)}

Atomic Force microscopy was performed on bare PET and ion treated samples in $30 \mu \mathrm{m}$ and 1 $\mu \mathrm{m}$ scales. Figure 4.7 displays the image of untreated PET film where the film average roughness in $30 \mu \mathrm{m}$ scale is measured to be $10.4 \mathrm{~nm}$. The images of PET surfaces after exposure to ion bombardment are also shown in Figure 4.8 to Figure 4.10. The results show that the samples with 30 min O-treatment have the highest roughness. Formation of new bonds after oxygen treatment could cause the microstructural change in PET and thus increases the surface roughness [93]. Nevertheless, with 60 min O-treatment, oxygen ions could break carbon-oxygen bonds and cause reduction in surface roughness down to close to that of bare PET, which would be the most effective treatment among all to change the surface structure to be appropriate for DLC deposition.

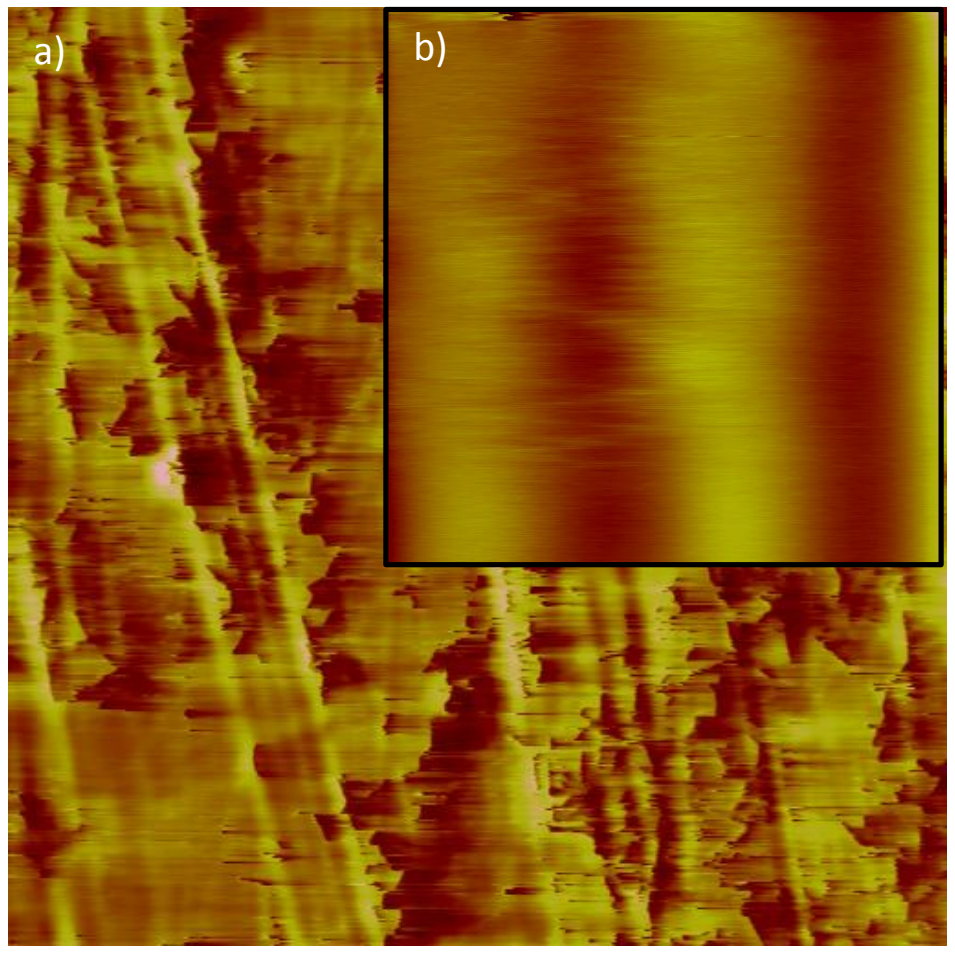

Figure 4.7. AFM images of bare PET in a) $30 \mu \mathrm{m}$ and b) $1 \mu \mathrm{m}$ scales 

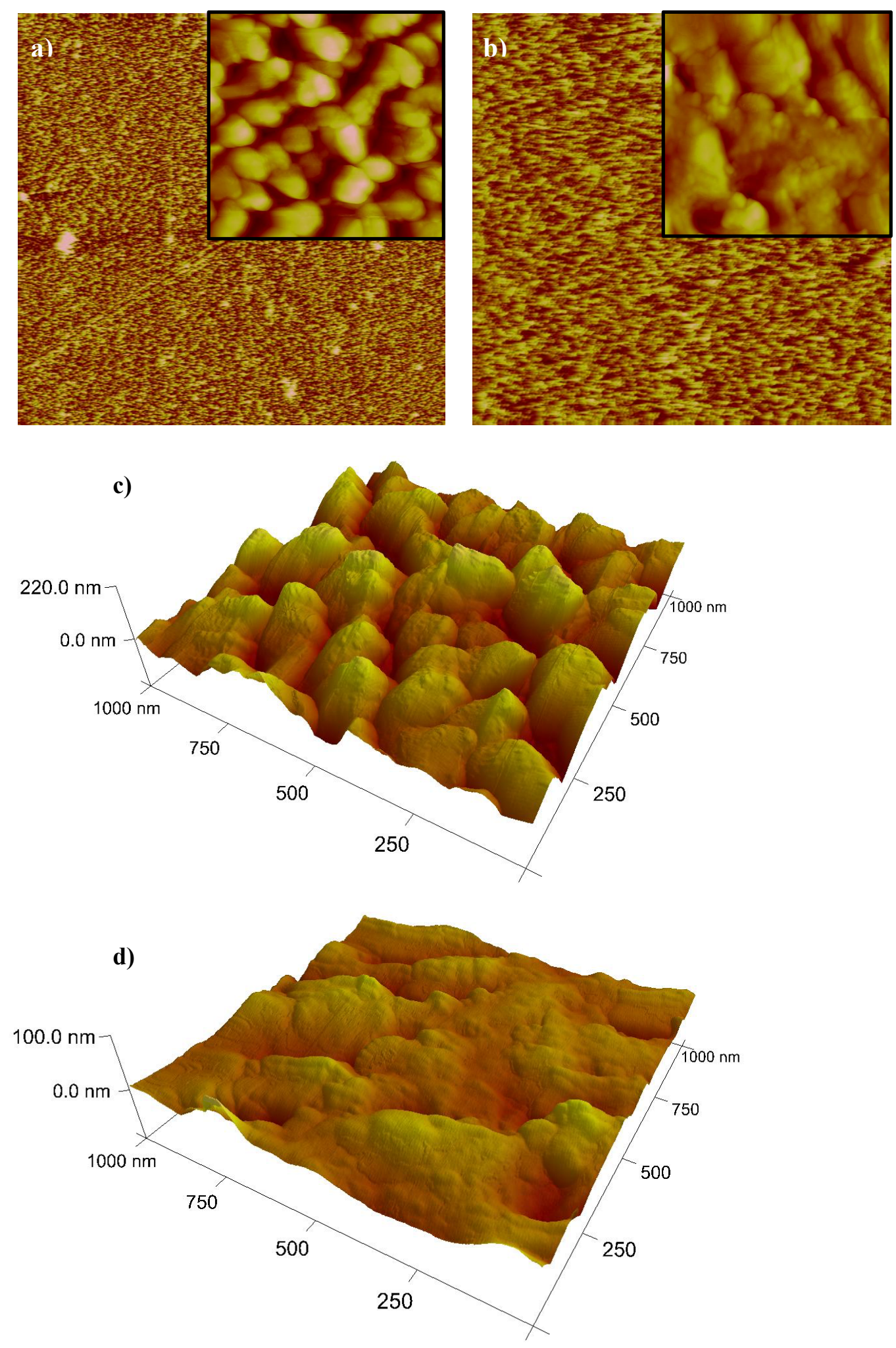

Figure 4.8. AFM images of PET films after oxygen ion treatment, a) 2D image after $30 \mathrm{~min}$ treatment, b) 2D image after 60 min treatment, c) 3D image after 30 min treatment, and d) 3D image after 60 min treatment 
On the contrary, 30 minutes nitrogen treatment dramatically decreases the roughness of PET with a finer microstructure comparing to oxygen treatment as shown in Figure 4.9. As observed by XPS, $\mathrm{N}$ ion treatment facilitated the formation of new $\mathrm{C}-\mathrm{N}$ bonds and free radicals on the surface. By increasing N-treatment time, the roughness decreases slightly with finer microstructure. The finest microstructure was observed on the PET surface after 60 min argon ion treatment (see Figure 4.10). As shown from XPS results, no new bonds have been observed in Ar-treated sample. Etching is probably the main mechanism for argon ion treatment, which roughens the surface by forming free radicals and strengthening PET crosslinking.
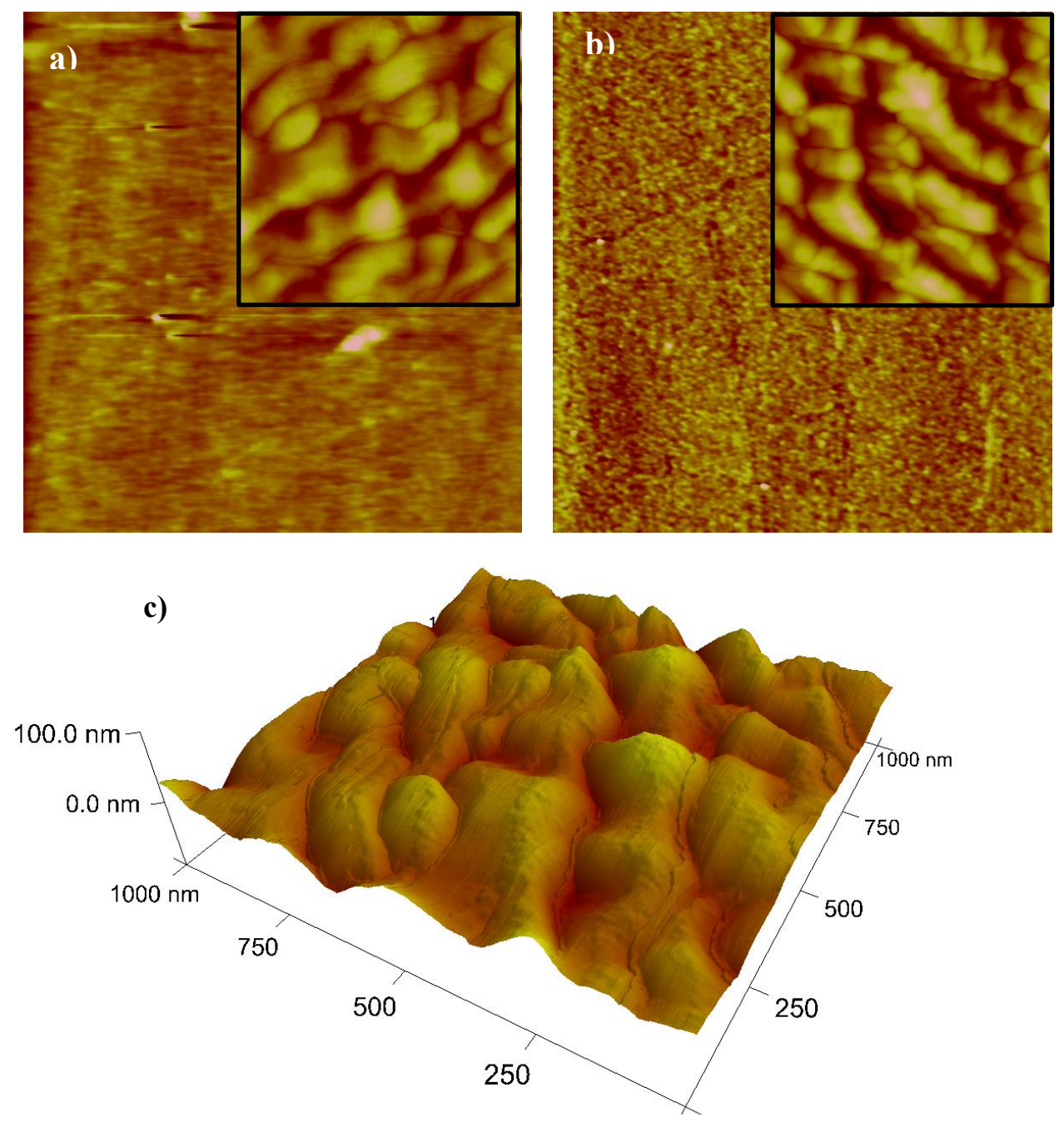


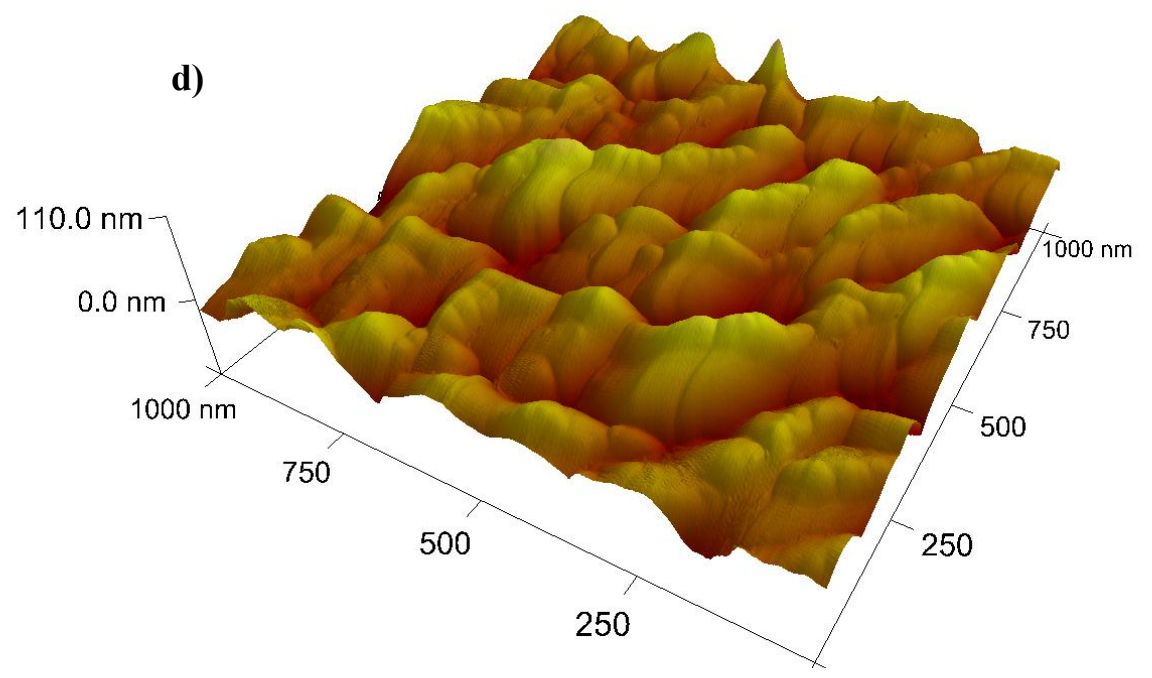

Figure 4.9. AFM images of PET surface after Nitrogen treatment, a) 2D image after $30 \mathrm{~min}$ treatment, b) 2D image after 60 min treatment, c) 3D image after 30 min treatment, and d) 3D image after 60 min treatment
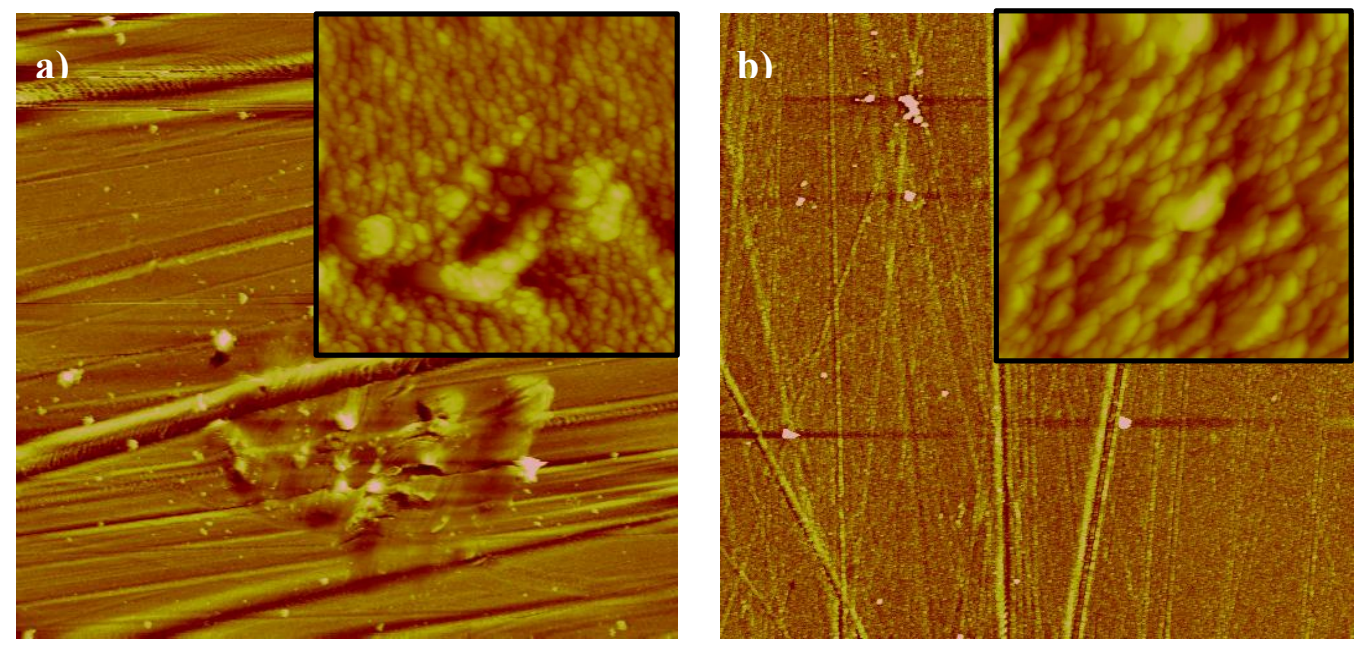

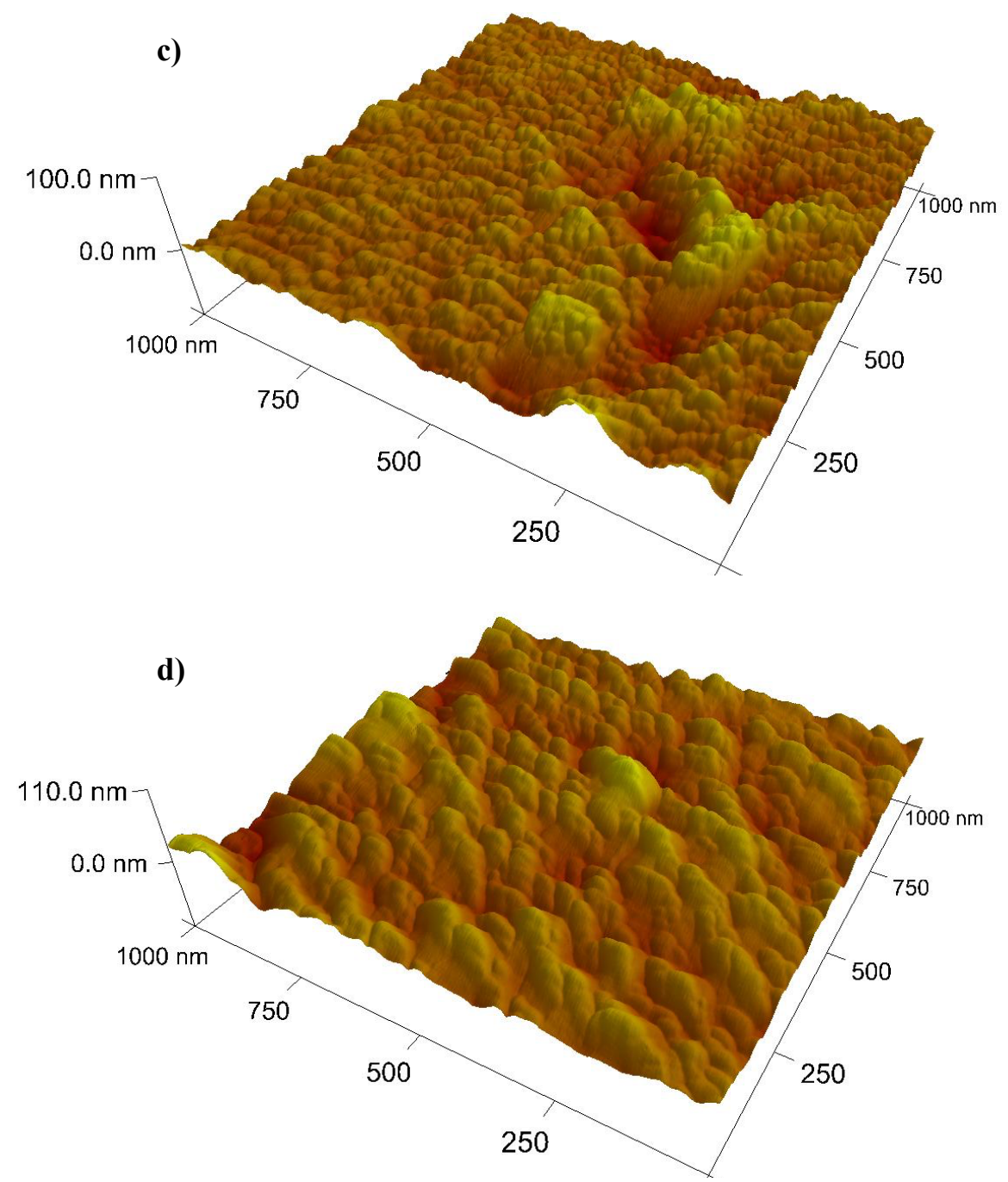

Figure 4.10. AFM images of PET surface after argon ion, a) 2D image after 30 min treatment, b) 2D image after 60 min treatment, c) 3D image after 30 min treatment, and d) 3D image after 60 min treatment

\subsubsection{Surface roughness}

Roughness measurements of PET films were also performed with optical profiler. The advantage of optical profiler over AFM is its ability to measure larger area. The average roughness values of all the samples are presented in Table 4.1, which are consistent with AFM results. 30 min Otreated samples show the highest roughness, which is followed by $30 \mathrm{~min}$ argon and nitrogen treated samples. The results show that shorter time treatment causes higher roughness for the same ions. Since there are some scratches on the surface of untreated PET films, the etching effect due to the long time treatment can smoothen the surface [94]. 
Table 4.3. Roughness, water contact angle, and surface energy values of plasma treated PET

\begin{tabular}{|c|c|c|c|}
\hline $\begin{array}{c}\text { Surface } \\
\text { treatment }\end{array}$ & $\begin{array}{c}\text { Roughness } \\
\text { nm }\end{array}$ & $\begin{array}{c}\text { Contact } \\
\text { Angle } \\
\left({ }^{\circ}\right)\end{array}$ & $\begin{array}{c}\text { Surface } \\
\text { Energy } \\
m N / m\end{array}$ \\
\hline Bare PET & $13 \pm 1$ & $81.2 \pm 0.1$ & $83.9 \pm 0.1$ \\
\hline $30-\mathrm{Ar} / \mathrm{O}_{2}$ & $21 \pm 1$ & $79.5 \pm 0.2$ & $86.1 \pm 0.2$ \\
\hline 60- $\mathrm{Ar} / \mathrm{O}_{2}$ & $13 \pm 1$ & $95.4 \pm 0.1$ & $66.0 \pm 0.1$ \\
\hline $30-A r / A r$ & $16 \pm 1$ & $93.5 \pm 0.2$ & $68.4 \pm 0.2$ \\
\hline $60-A r / A r$ & $10 \pm 1$ & $86.7 \pm 0.2$ & $77.0 \pm 0.3$ \\
\hline $30-A r / N_{2}$ & $14 \pm 1$ & $96.8 \pm 0.1$ & $64.2 \pm 0.1$ \\
\hline $60-A r / N_{2}$ & $11 \pm 1$ & $77.5 \pm 0.3$ & $88.5 \pm 0.4$ \\
\hline
\end{tabular}

\subsubsection{Contact angle and surface energy}

Water contact angle of PET film after different ion treatments were measured with goniometer and the surface energies were then calculated based on the measured contact angles. Both the contact angles and the surface energies are listed in table 4.3. Contact angle of bare PET is $81.2^{\circ}$ and the calculated surface energy is $83.9 \mathrm{mN} / \mathrm{m}$. After 30 minute exposure to oxygen ions and 60 minutes nitrogen ions, the contact angle decreases to $79.5^{\circ}$ and $77.5^{\circ}$, respectively. Other ion treatment conditions result in increasing contact angle and hydrophobicity. The highest contact angle belongs to $30 \mathrm{~min} \mathrm{~N}$-treatment followed by $60 \mathrm{~min}$ O-treatment and $30 \mathrm{~min}$ Ar-treatment as presented in Table 4.3 and Figure 4.11. The results show that both ion types and treatment time change surface energy as formation of polar groups such as $-\mathrm{OH}, \mathrm{CO}, \mathrm{COOH}$ and $\mathrm{COO}-$ on the surface could enhance hydrophilic properties compared to bare PET [95]. In addition, increasing the roughness could result in higher surface energy and chemical composition and bonding structure changes could also play role. 


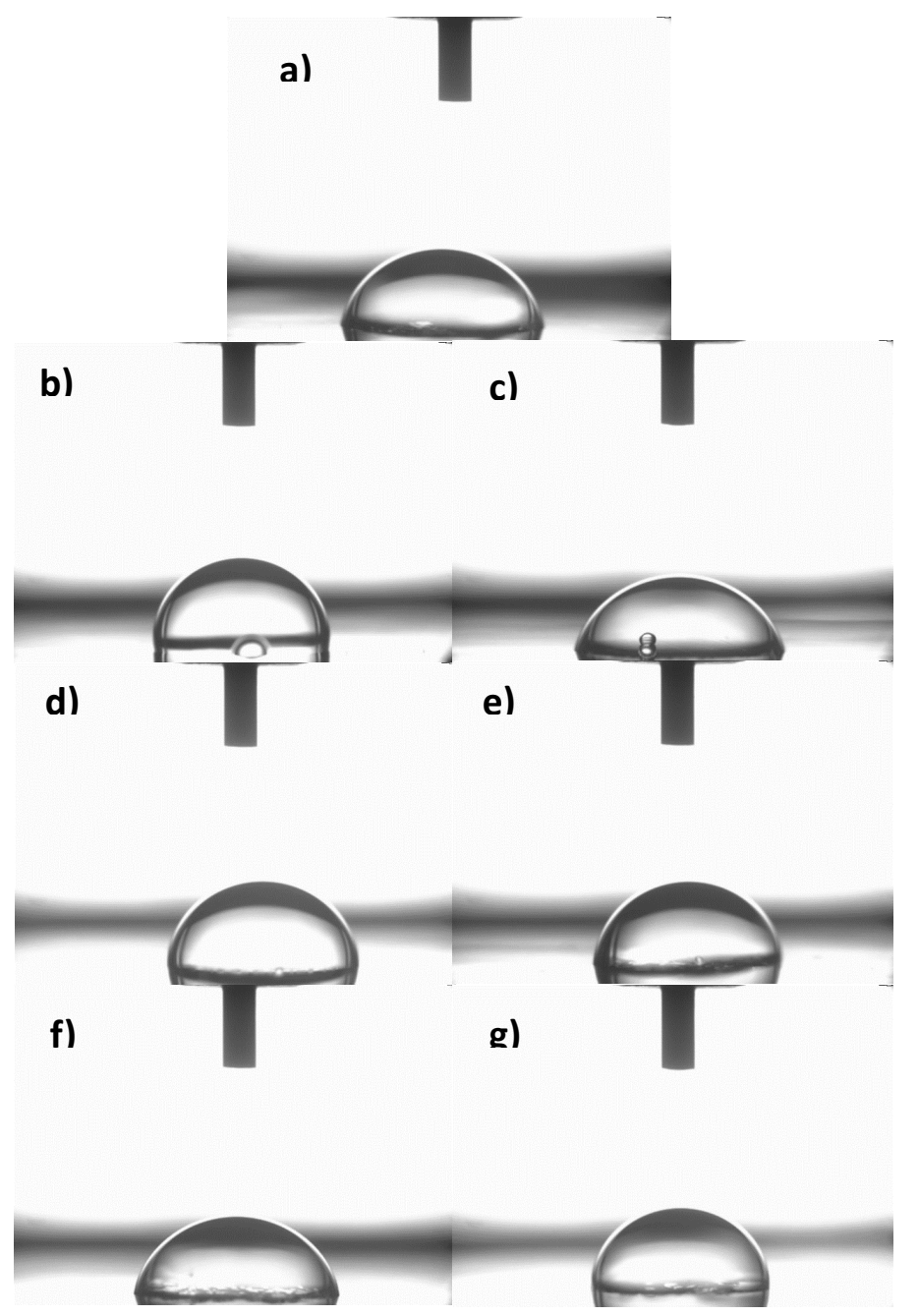

Figure 4.11. Water contact angel of a) bare PET, b) 30 min N-treatment, c) 60 min N-treatment, d) $30 \mathrm{~min}$ Ar-treatment, e) $60 \mathrm{~min}$ Ar-treatment, f) $30 \mathrm{~min}$ O-treatment, and g) $60 \mathrm{~min} \mathrm{O}-$ treatment

\subsubsection{Friction and wear}

The COF for different samples is summarized in Figure 4.12. It shows that COF of samples after treatment is higher than to the bare PET samples. 60 minute Ar-treated samples have the highest COF whereas 30 minutes O-treated samples show the lowest COF although 60 min Ar-treated samples have the smoothest surface and 30 minutes O-treated ones have the roughest surface. The results show that the chemical composition and bonding structure of PET surface more important role than the surface roughness in controlling COF in this case. It seems that the dangling bonds formed by Ar ion bombardment cause higher COF [96]. 


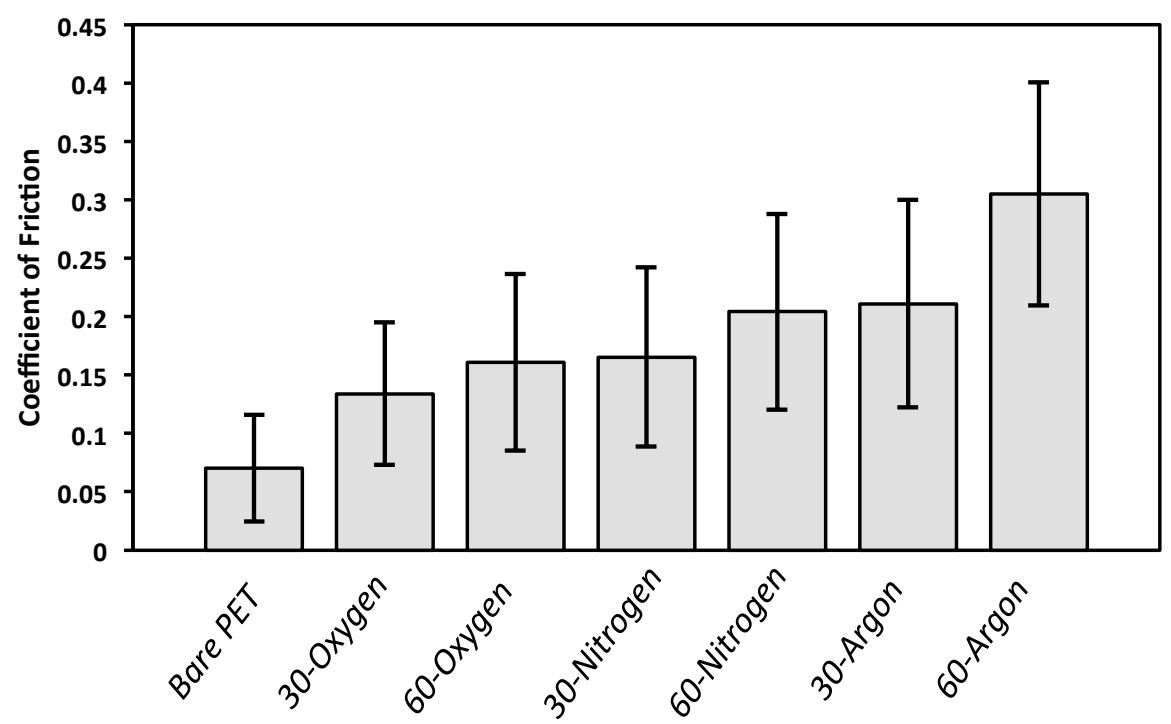

Figure 4.12. COF of untreated and ion treated PET films 


\subsection{Effect of ion treatment of PET substrate films on DLC deposition}

\subsubsection{Raman spectroscopy}

A typical Raman spectrum of DLC films consists of two broad peaks: a disordered D peak centered around $1360 \mathrm{~cm}^{-1}$ and a graphitic $\mathrm{G}$ peak around $1575 \mathrm{~cm}^{-1}$. Since $\pi$ states ( $\mathrm{sp}^{2}$ bonds) have lower bond energy than $\sigma$ states ( $\mathrm{sp}^{3}$ bonds), $\pi$ states can be easily polarized to have much larger Raman scattering cross section than $\sigma$ states. Furthermore, $G$ peak is sensitive to the stretching vibration of $\mathrm{sp}^{2}$ sites in either $\mathrm{C}=\mathrm{C}$ chain or aromatic ring while $\mathrm{D}$ peak is only sensitive to breathing mode of $\mathrm{sp}^{2}$ sites in the ring [27]. Therefore, visible Raman spectrum can give valuable information on the bonding states of DLC by analyzing the position and full width of half maximum (FWHM) of the G peak and the intensity ratio of the D peak to G peak [16]. The Raman spectra of DLC-M (using methane as carbon source) and DLC-A (using acetylene as carbon source) are displayed in Figure 4.13 and the $\mathrm{I}_{\mathrm{D}} / \mathrm{I}_{\mathrm{G}}$ ratio is calculated and the ratio is 0.82 for DLC deposited with methane gas and 1.31 for acetylene as precursor gas, showing higher in $\mathrm{sp}^{2}$ bonding content in using $\mathrm{C}_{2} \mathrm{H}_{2}$. Do et al. [97] reported that it was very difficult to form C-C single bonds in acetylene plasma because hydrocarbon gases with even numbers of carbons are more likely to form double $\left(\mathrm{sp}^{2}\right)$ bonds than single $\left(\mathrm{sp}^{3}\right)$ bonds in the deposited DLC films.

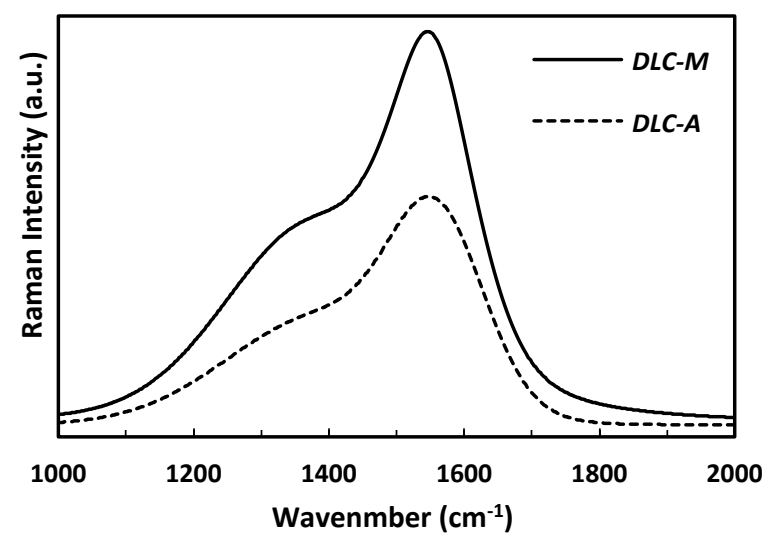

Figure 4.13. Raman spectra of DLC-M and DLC-A

\subsubsection{X-ray photoelectron spectroscopy (XPS)}

The curve-fitted XPS spectra of C1s in DLC films deposited in two different precursor gases of $\mathrm{CH}_{4}$ and $\mathrm{C}_{2} \mathrm{H}_{2}$ are shown in Figure 4.14. The peak was de-convoluted with CasaXPS software using Gaussian/Lorentzian function after background removal using Shirley method into two 
peaks; $\mathrm{sp}^{2}$ carbon at a lower binding energy of $284.4 \pm 0.1 \mathrm{eV}$ and $\mathrm{sp}^{3}$ carbon at a higher binding energy of $285.1 \pm 0.1 \mathrm{eV}$. The shoulder at higher binding energy is attributed to carbons atoms bonded with oxygen on the film surface [98]. By measuring the area of the de-convoluted $\mathrm{sp}^{2}$ and $\mathrm{sp}^{3}$ peaks after curve fitting, the area ratio of the peaks $\left(\mathrm{I}_{\mathrm{C}=\mathrm{C}} / \mathrm{I}_{\mathrm{C}-\mathrm{C}}\right)$ is determined to be 1.31 and 2.88 for DLC-M and DLC-A, respectively. The same trend was also found with Raman analysis that confirms that $\mathrm{C}_{2} \mathrm{H}_{2}$ precursor gas facilitates $\mathrm{sp}^{2}$ formation in the deposited DLC films.
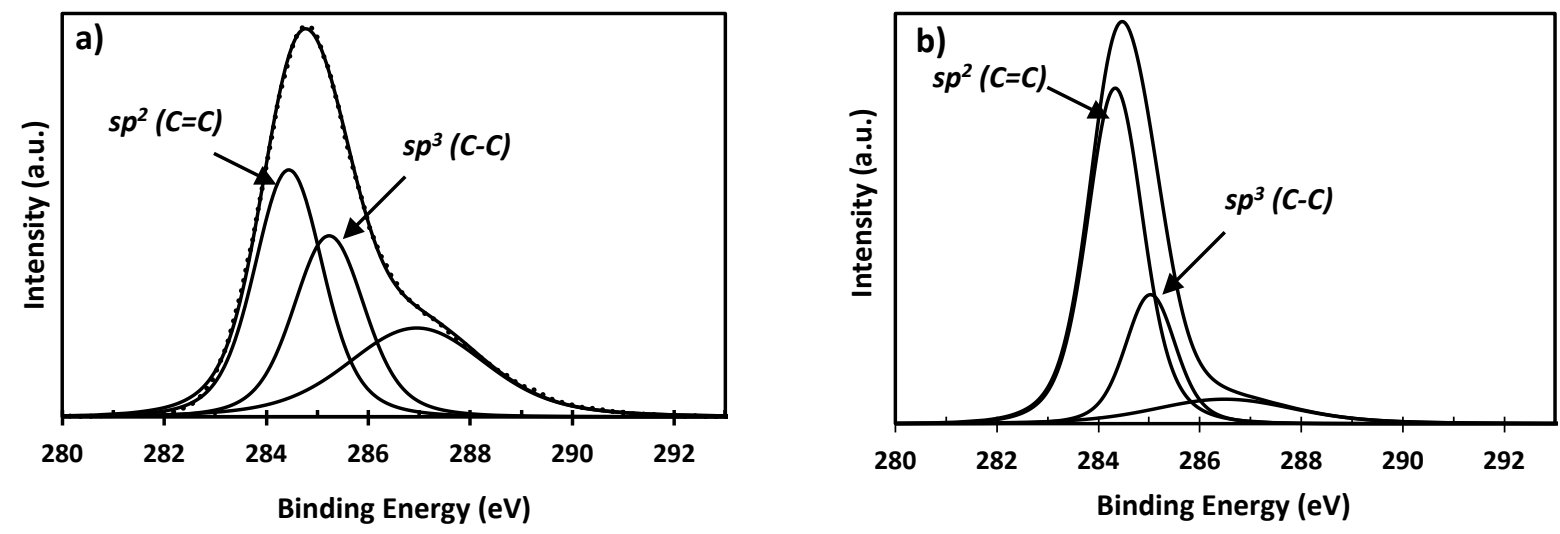

Figure 4.14. C1s XPS spectra of DLC sample deposited on PET, a) DLC-M, b) DLC-A

\subsubsection{Roughness measurement}

Table 4.4 summarizes the average roughness values for bare PET, DLC-M and DLC-A deposited on ion treated PET films measured by the optical profiler. The surface roughness of PET before deposition is $12.9 \mathrm{~nm}$ and increases dramatically after DLC deposition. Pure DLC deposited with Methane gas has the highest roughness. Ion treatment of the substrates reduces the roughness, where O-treatment is most effective for reduction of roughness. Furthermore, the longer the treatment, the lower the roughness, the lowest roughness values are achieved for DLC films deposited on 60 min O-treated PET. This is probably due to the etching, crosslinking, and surface activation (radical formation) effects caused by ion bombardment, which enhances the interaction between the PET substrate and the DLC film [99]. 
Table 4.4. Roughness values of DLC-M and DLC-A deposited on ion treated films

\begin{tabular}{|c|c|}
\hline Surface treatment & $\begin{array}{c}\text { Roughness } \\
\mathrm{nm}\end{array}$ \\
\hline Bare PET & $13 \pm 1$ \\
\hline$D L C-M$ & $143 \pm 1$ \\
\hline$D L C-M+30-A r / O_{2}$ & $76 \pm 1$ \\
\hline$D L C-M+30-A r / N_{2}$ & $95 \pm 1$ \\
\hline$D L C-M+30-A r / A r$ & $102 \pm 1$ \\
\hline$D L C-M+60-A r / O_{2}$ & $27 \pm 1$ \\
\hline$D L C-M+60-A r / N_{2}$ & $62 \pm 1$ \\
\hline$D L C-M+60-A r / A r$ & $87 \pm 1$ \\
\hline$D L C-A$ & $102 \pm 1$ \\
\hline$D L C-A+60-A r / O_{2}$ & $36 \pm 1$ \\
\hline$D L C-A+60-A r / N_{2}$ & $71 \pm 1$ \\
\hline$D L C-A+60-A r / A r$ & $74 \pm 1$ \\
\hline
\end{tabular}

\subsubsection{Contact angle and surface energy}

Water contact angle of PET film, DLC-M and DLC-A were measured and shown in Figure 4.15. Contact angle of bare PET is about $81.2^{\circ}$ and the surface energy calculated is $83.9 \mathrm{mN} / \mathrm{m}$. The contact angle of DLC-M coated PET is $71.3^{\circ}$ and the calculated surface energy is $96.0 \mathrm{mN} / \mathrm{m}$. For DLC coated PET with $\mathrm{C}_{2} \mathrm{H}_{2}$, the water contact angle is $67.5^{\circ}$ and calculated energy increased to $100.7 \mathrm{mN} / \mathrm{m}$. Increase of surface energy because DLC deposition is probably due to the increased roughness caused by ion bombardment.

a)
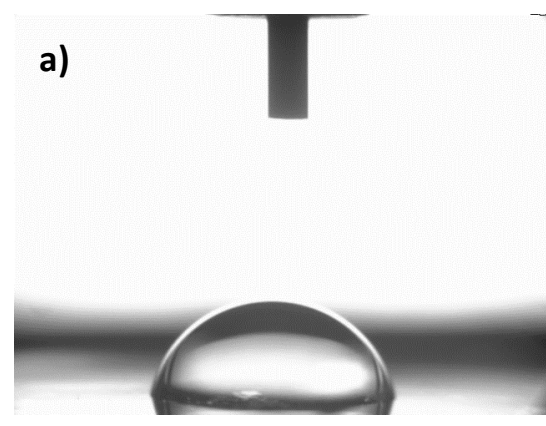

b)
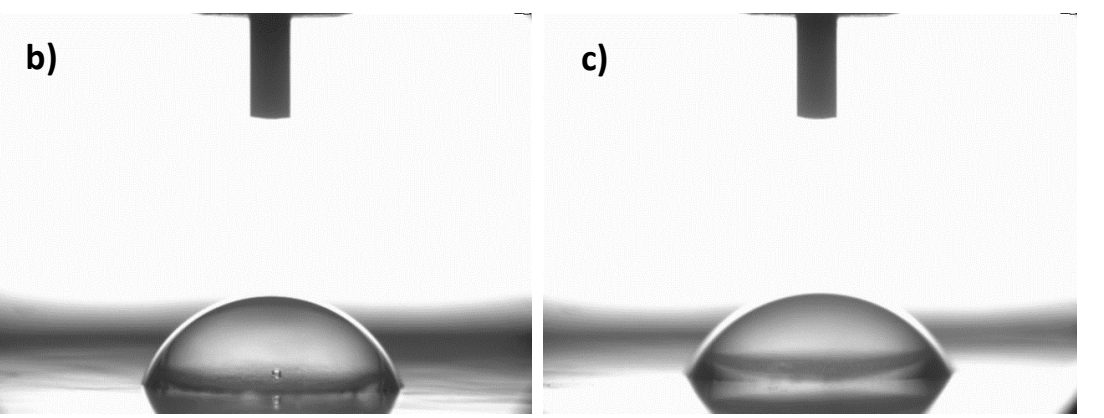

Figure 4.15. Water contact measurements of a) bare PET, b) DLC-M, and c) DLC-A 


\subsubsection{Scratching results}

Figure 4.16 illustrates optical microscopic images obtained from on DLC coated samples after scratching test with an increasing load from 0.1 to $0.5 \mathrm{mN}$. In this study, all the DLC depositions were performed under same deposition conditions and thus the properties of fabricated coatings such as hardness and COF are same in all the samples. Therefore, based on the crack width and observation of delamination around the scratch line, adhesion strength between DLC and polymer as a soft substrate could be understood. We can see that no film delamination for 60 minutes O-treated samples with methane as gas precursor, partially delamination for 30 minutes O-treated samples, and full delamination for other cases. The width of scratch is lower for oxygen treatment samples, which is followed by nitrogen treated ones, where argon treatment shows least influence. Furthermore, by comparing Figure 4.16.b with Figure 4.16.c, we can see that the width of scratch on 60 minutes O-treated samples is about $11.01 \mu \mathrm{m}$, which is less than that $(12.99 \mu \mathrm{m})$ for 30 minutes O-treated samples. Comparing Figure 4.16.c with 4.16.f, we can see that the scratch width on DLC-A is almost two times as that on DLC-M. The results show that O-treatment is effective in enhancing DLC adhesion. This is probably because the formation of free radicals on the PET surface after oxygen treatment. 

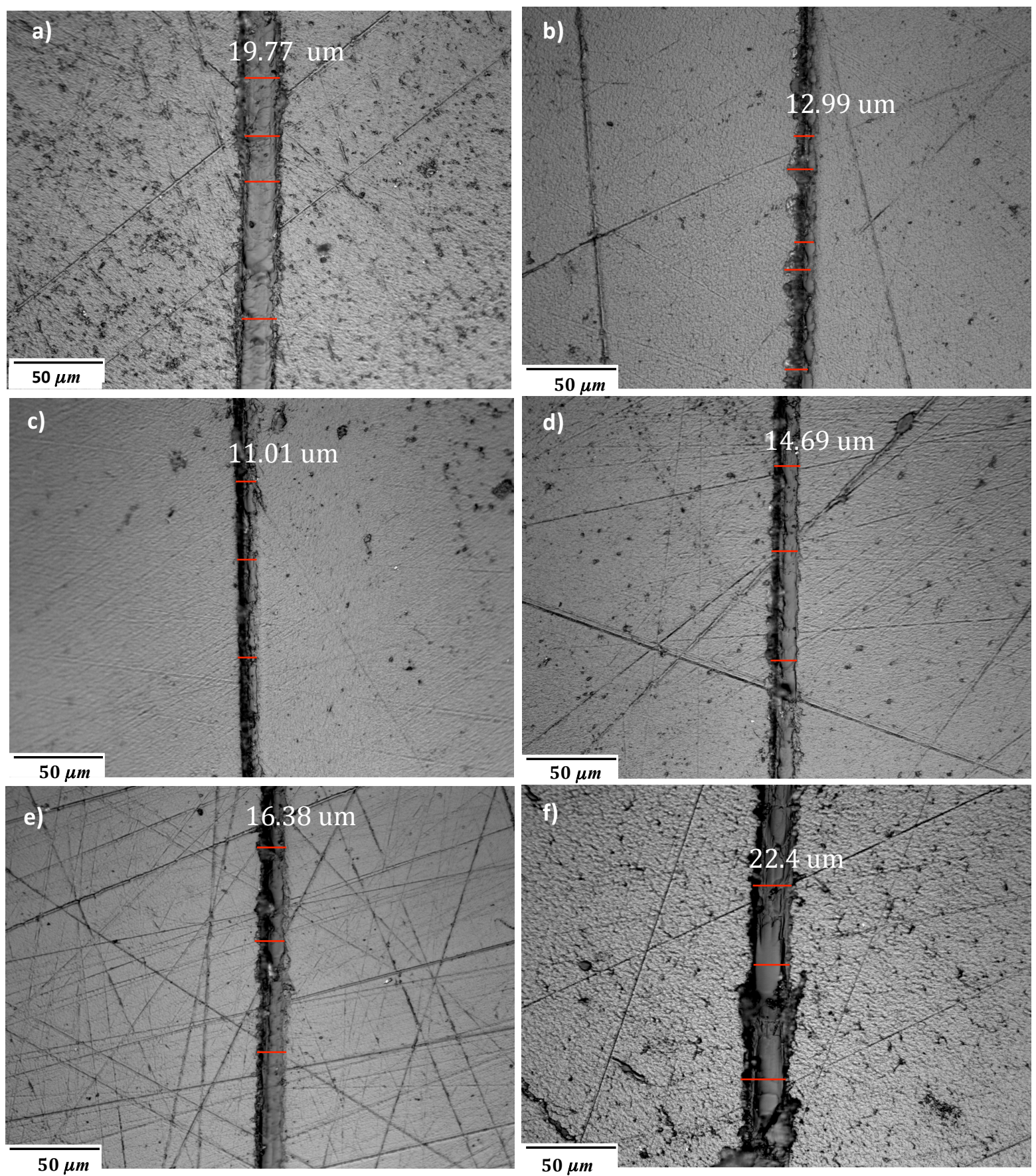

Figure 4.16. Width measurement of scratch line a) DLC-M+30 min N-treatment, b) DLC-M+30 min O-treatment, c) DLC-M+60 min O-treatment, d) DLC-M+60 min N treatment, e) DLC$\mathrm{M}+60$ min Ar-treatment, and f) DLC-A +60 min O-treatment 


\subsection{Effect of doping on the structure and properties of DLC on PET}

\subsubsection{Raman spectroscopy}

The Raman spectra of N-DLC and Si-DLC with different concentrations are presented in Figure 4.17. For N-DLC, the $\mathrm{G}$ peak position shifts to a higher value, and the higher the $\mathrm{N}$ concentration, the higher the shift of the G peak whereas for Si-DLC, G peak position shifts to lower numbers and the higher the Si concentration, the lower the shift of the G peak. The change in $\mathrm{G}$ peak position is closely related to $\mathrm{sp}^{2} / \mathrm{sp}^{3}$ fraction in the coating. It has been reported that $\mathrm{Si}$ doping into DLC enhances $\mathrm{sp}^{3}$ bonding which moves the $\mathrm{G}$ peak to a lower value whereas $\mathrm{N}$ doping into DLC facilitates the formation of $\mathrm{sp}^{2}$ bonding and thus shifts $\mathrm{G}$ peak to a higher value [100]. Based on the Raman spectra shown in Figure 4.17, $\mathrm{I}_{\mathrm{D}} / \mathrm{I}_{\mathrm{G}}$ ratio is calculated. The ratio is 0.82 for Pure DLC, 1.12, 1.40, and 1.42 for N-DLC-1, N-DLC-2, and N-DLC-3, respectively, further showing that confirm doping enhances $\mathrm{sp}^{2}$ bonding formation. However, $\mathrm{I}_{\mathrm{D}} / \mathrm{I}_{\mathrm{G}}$ ratio for Si-DLC samples is 0.76 for Si-DLC-400 and 0.54 for Si-DLC-800, confirming Si doping facilitates the formation of $\mathrm{sp}^{3}$ bonding. This is due to the fact that $\mathrm{Si}$ tends to form $\mathrm{sp}^{3}$ four-fold coordinated networks. Furthermore, the intensity of D peak decreases by incorporation of silicon and increases by nitrogen doping. This is because the intensity of D peak is sensitive to the breathing mode of $\mathrm{sp}^{2}$ sites. The results indicate that the number of $\mathrm{sp}^{2}$ rings per cluster is enhanced by nitrogen doping while silicon causes creation of highly disordered $\mathrm{sp}^{2}$ sites and thus reduces the size of $\mathrm{sp}^{2}$-bonded clusters, consistent with the results reported previously [101103].

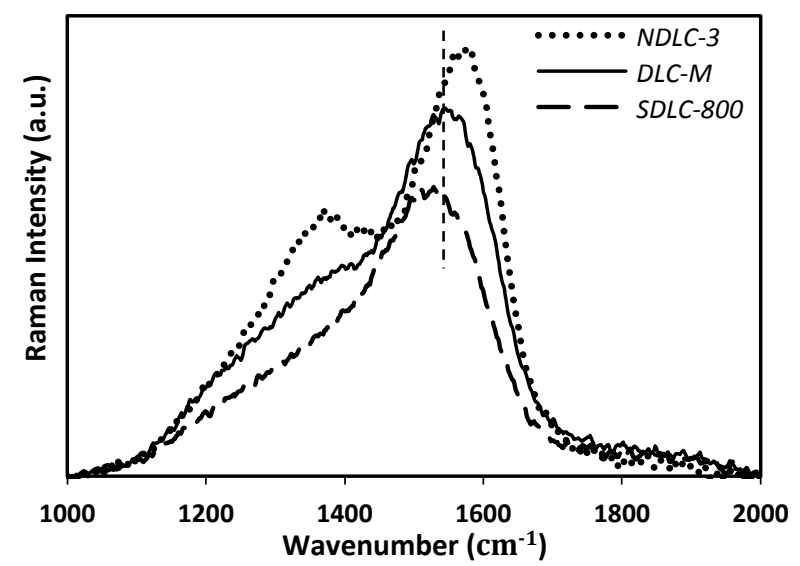

Figure 4.17. Raman spectra of DLC-M, NDLC-3 and SDLC-800 


\subsubsection{Fourier transform infrared spectroscopy (FTIR)}

Figure 4.18 depicts FTIR spectra of bare PET, DLC, N-DLC-3 and Si-DLC-800 deposited on PET. The main absorption bands of $1720 \mathrm{~cm}^{-1}$ is attributed to the ester carbonyl bond stretching $(\mathrm{C}=\mathrm{O}), 1247 \mathrm{~cm}^{-1}$ to the asymmetric $\mathrm{C}-\mathrm{C}-\mathrm{O}$ stretching and $1100 \mathrm{~cm}^{-1}$ to the methylene group $[104,105]$. The shift of these bands to the lower value after DLC deposition is clearly shown by the vertical line in Fig. 4.18.a, indicating the formation of new bonding between the coating and the PET substrate. Furthermore, the intensity ratio of carbonyl $\mathrm{C}=\mathrm{O}$ peak to asymmetric $\mathrm{C}-\mathrm{C}-\mathrm{O}$ stretching peak is reduced after DLC deposition. Figure 4.18.b shows the change in aromatic and aliphatic $\mathrm{C}-\mathrm{H}$ bond stretching at $3100-2800 \mathrm{~cm}^{-1}$. Different C-H absorption bands in the samples suggest structural differences in DLC samples with different composition.

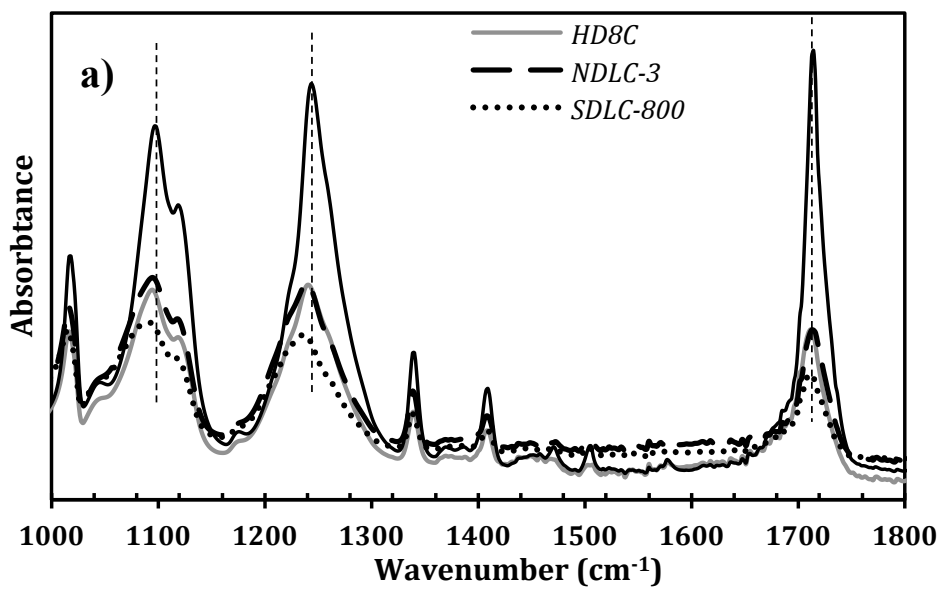

b)

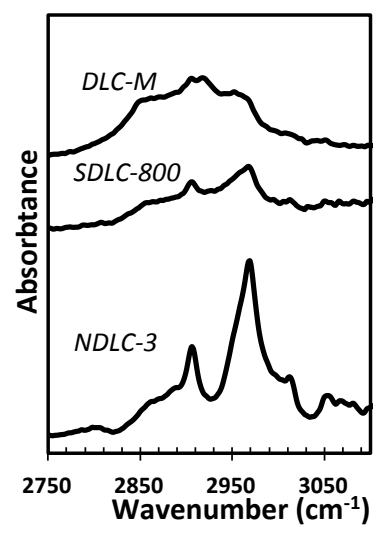

Figure 4.18. a) ATR-FTIR Spectra of bare PET, DLC-M, NDLC-3 and SDLC-800 coated PET

b) C-H stretching vibration mode of DLC-M. NDLC-3 and SDLC-800 on PET

\subsubsection{X-ray photoelectron spectroscopy (XPS)}

Figure 4.19 displays wide scan XPS spectra of the samples. Intense photoemission peaks at C1s, and O1s were detected for all of the samples including DLC-M, NDLC-3, and SDLC-800 while N1s peak was only detected for Nitrogen doped DLC and Si2s and Si2p peaks were observed in silicon doped DLC samples as expected. Studying the detected spectra could give detail information about the chemical structural and bonding states of the samples. The silicon and nitrogen content can be calculated from the ratios of the areas of N1s and sum area of Si2s and Si2p peaks to the total peak areas with the consideration of atomic sensitivity factor. As results 
shown in Table 4.5, the nitrogen content increased by increasing the nitrogen, respectively. Increasing nitrogen flow rate during the film deposition or increasing the target bias of $\mathrm{Si}$ will increase $\mathrm{N}$ and $\mathrm{Si}$ content, respectively.

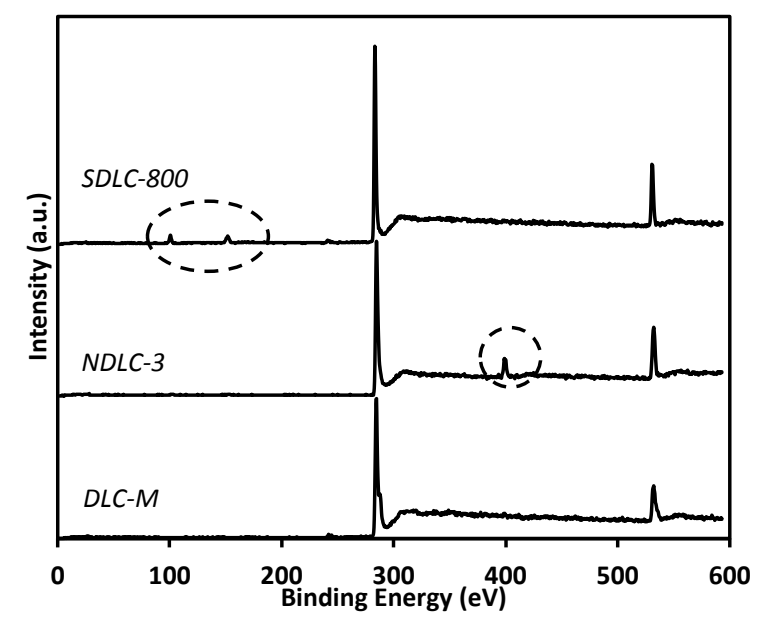

Figure 4.19. Wide scan Spectra of pure DLC, N-DLc and Si-DLC

Table 4.5. Bonding state of DLC based coatings

\begin{tabular}{|c|c|c|c|c|c|c|c|c|}
\hline & $N \%$ & $S i \%$ & $C \%$ & $O \%$ & $\begin{array}{l}s p^{2}(C=C) / \\
s p^{3}(C-C)\end{array}$ & $\begin{array}{c}s p^{2} \\
([C=C]+[C=N]) / \\
s p^{3}([C-C]+[C-N])\end{array}$ & $\begin{array}{c}s p^{2} \\
(C=N) / \\
s p^{3}(C-N)\end{array}$ & $\begin{array}{c}s p^{2}([C=C]) / \\
s p^{3}([C- \\
C]+[S i-C])\end{array}$ \\
\hline$D L C-M$ & - & - & 83.0 & 12.6 & 1.3 & - & - & - \\
\hline$N D L C-1$ & 4.4 & - & 81.8 & 13.4 & 2.3 & 2.1 & 0.9 & - \\
\hline$N D L C-2$ & 4.8 & - & 80.8 & 12.2 & 2.5 & 2.6 & 1.5 & - \\
\hline$N D L C-3$ & 7.0 & - & 87.6 & 10.5 & 4.7 & 4.1 & 2.1 & - \\
\hline$S D L C-400$ & - & 1.9 & 81.4 & 11.36 & 1.2 & - & - & 0.9 \\
\hline$S D L C-800$ & - & 4.1 & 83.0 & 12.62 & 0.5 & - & - & 0.4 \\
\hline
\end{tabular}

Figure 4.20 illustrates the C1s XPS spectra of nitrogen doped DLC samples with different nitrogen concentrations. Five peaks exhibit in de-convoluted spectra at $284.4 \pm 0.1 \mathrm{eV}, 285.1 \pm 0.1$ $\mathrm{eV}, 285.7 \pm 0.1 \mathrm{eV}, 286.7 \pm 0.1 \mathrm{eV}$, and $288.3 \pm 0.1 \mathrm{eV}$, which are attributed to $\mathrm{C}=\mathrm{C}, \mathrm{C}-\mathrm{C}, \mathrm{C}=\mathrm{N}, \mathrm{C}-$ $\mathrm{N}$, and C-O, respectively $[98,106]$. According to the peak area of de-convoluted peaks, $\mathrm{sp}^{2} / \mathrm{sp}^{3}$ ratios of N-DLC samples were calculated based on $\mathrm{sp}^{2}([\mathrm{C}=\mathrm{C}]+[\mathrm{C}=\mathrm{N}]) / \mathrm{sp}^{3}([\mathrm{C}-\mathrm{C}]+[\mathrm{C}-\mathrm{N}])$ and 
$\mathrm{sp}^{2}([\mathrm{C}=\mathrm{C}]) / \mathrm{sp}^{3}([\mathrm{C}-\mathrm{C}])$ and are presented in Table 4.5. As it can be seen, the area under $\mathrm{C}=\mathrm{C}$ and $\mathrm{C}=\mathrm{N}$ peaks increases and thus the $\mathrm{sp}^{2} / \mathrm{sp}^{3}$ ratio increases by increasing the nitrogen content, consistent with the Raman results. These results suggest that the nitrogen doped into the films exists in the form of $\mathrm{sp}^{2}$ configuration. Figure 4.21 shows de-convoluted XPS N1s spectra of NDLC samples. By increasing the nitrogen flow rate, the fraction of $\mathrm{C}=\mathrm{N}$ bonds to $\mathrm{C}-\mathrm{N}$ bonds increases (see Table 4.5), which indicates that the doped nitrogen promotes graphitic bonds. Similar results were reported by other researchers $[106,107]$
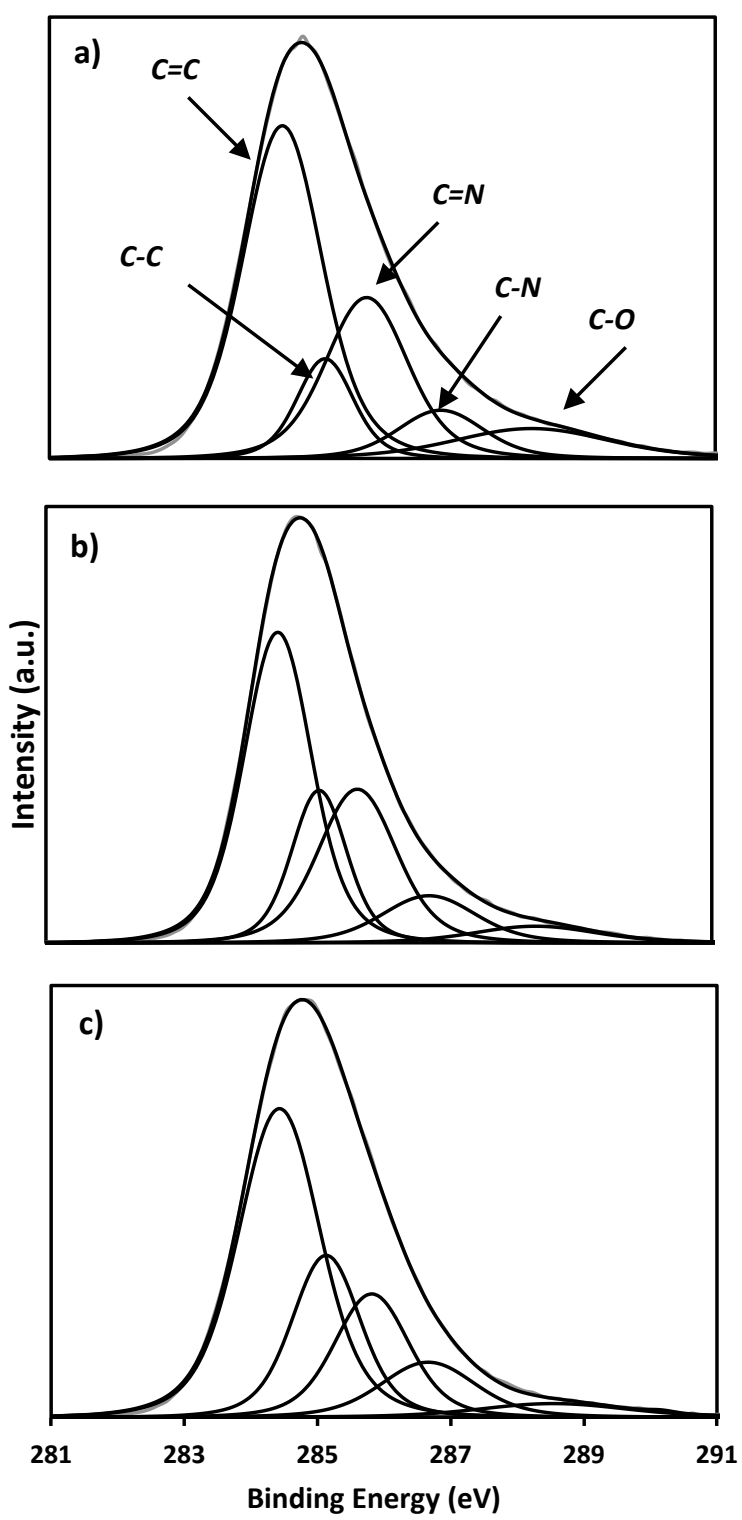

Figure 4.20. De-convolution of C1s XPS spectra of a) NDLC-3, b) NDLC-2 and c) NDLC-1 
Si-DLC XPS C1s spectra are indicated in Figure 4.22. The C1s peak can be de-convoluted into four peaks at $283.8 \pm 0.2 \mathrm{eV}, 284.5 \pm 0.1 \mathrm{eV}, 285.1 \pm 0.1 \mathrm{eV}$ and $287.2 \pm 0.2 \mathrm{eV}$, attributed to Si-C, $\mathrm{C}=\mathrm{C}, \mathrm{C}-\mathrm{C}$ and $\mathrm{C}-\mathrm{O}$ bonds, respectively $[108,109]$. The ratios of $\mathrm{sp}^{2}([\mathrm{C}=\mathrm{C}]) / \mathrm{sp}^{3}([\mathrm{C}-\mathrm{C}]+[\mathrm{Si}-\mathrm{C}])$ and $\mathrm{sp}^{2}([\mathrm{C}=\mathrm{C}]) / \mathrm{sp}^{3}([\mathrm{C}-\mathrm{C})]$ are listed in Table 4.5. The results show that the $\mathrm{sp}^{2} / \mathrm{sp}^{3}$ ratio decreased by increasing the silicon content, in agreement with Raman results, indicating that silicon atoms replace carbon atoms to form $\mathrm{sp}^{3} \mathrm{Si}-\mathrm{C}$ bonds. By analyzing Si2p XPS spectra displayed in Figure 4.23, It has been found that $\mathrm{Si}-\mathrm{C}$ content increases by increasing the target bias and the peaks of SDLC-400 are more symmetric and centered around $101 \mathrm{eV}$ while the peaks of SDLC-800 are more asymmetrical with higher shift shoulder $[109,110]$.

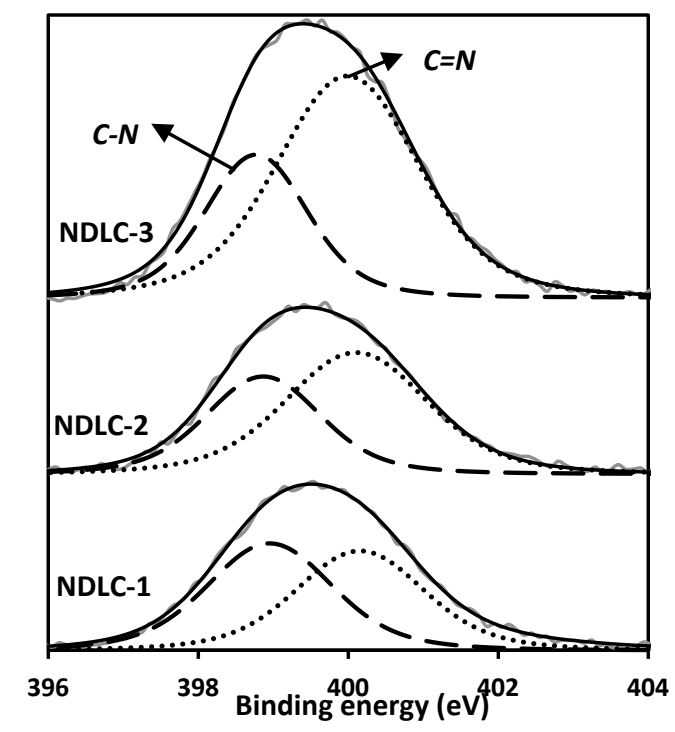

Figure 4.21. N1s XPS spectra of Nitrogen doped DLC samples with different N concentrations

\subsubsection{Near edge X-ray absorption fine structure (NEXAFS)}

Carbon K edge NEXAFS spectra of Nitrogen doped DLC samples in total electron yield (TEY) and fluorescence yield (FLY are presented in Figure 4.24 and Figure 4.25, respectively. TEY spectra are more surface sensitive whereas FLY spectra give information about both surface and inner layer. In TEY spectra, there are three main peaks around $285 \mathrm{eV}, 288 \mathrm{eV}$, and $292 \mathrm{eV} \mathrm{d}$. The first peak around $285 \mathrm{eV}$ is attributed to transition of electron from $\mathrm{C} 1 \mathrm{~s}$ to $\pi^{*}$ of $\mathrm{sp}^{2} \mathrm{C}=\mathrm{C}$ bond while the small peak about $288 \mathrm{eV}$ is because of $\mathrm{sp}^{3} \mathrm{C}-\mathrm{H}$ bonds, justifying presence of carbon dangling bonds on the surface passivated by hydrogen. The peak at $292 \mathrm{eV}$ can be 
attributed to transition of $\mathrm{C} 1 \mathrm{~s}$ electrons to $\sigma^{*}, \mathrm{sp}^{3} \mathrm{C}-\mathrm{C}$, bonds [111]. A very low intensity peak is appeared around $287.5 \mathrm{eV}$, which is attributed to $\mathrm{C}=\mathrm{O} \pi^{*}$ bonding, probably because of the exposure to atmosphere $[32,111]$.
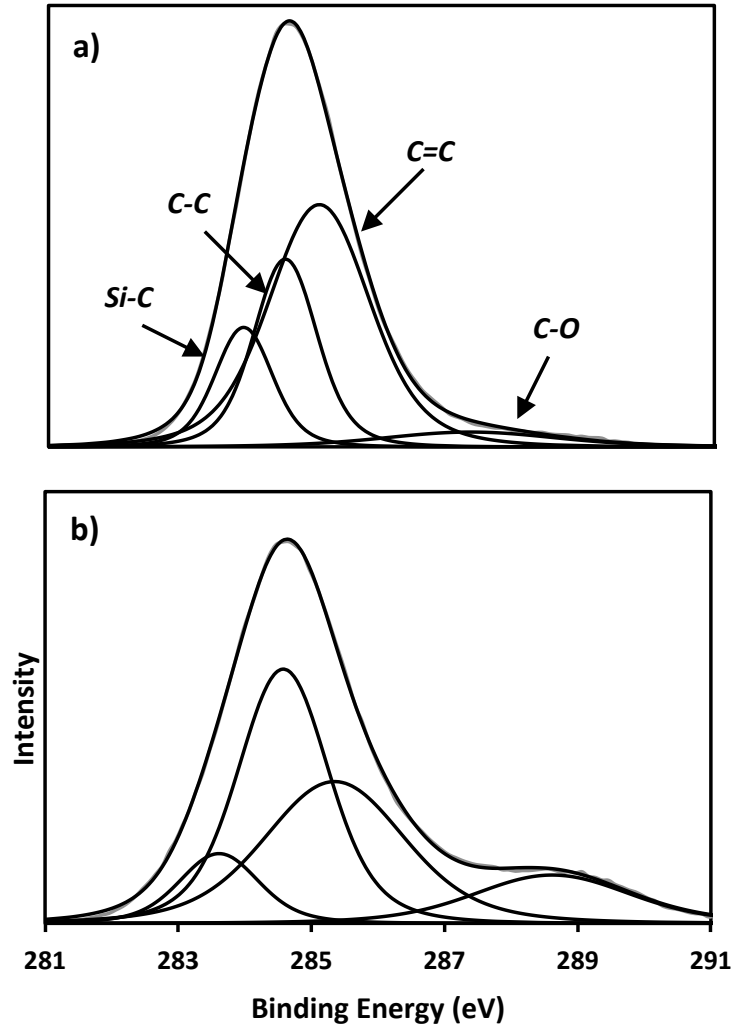

Figure 4.22. De-convolution of C1s XPS spectra of a) SDLC-800, b) SDLC-400 


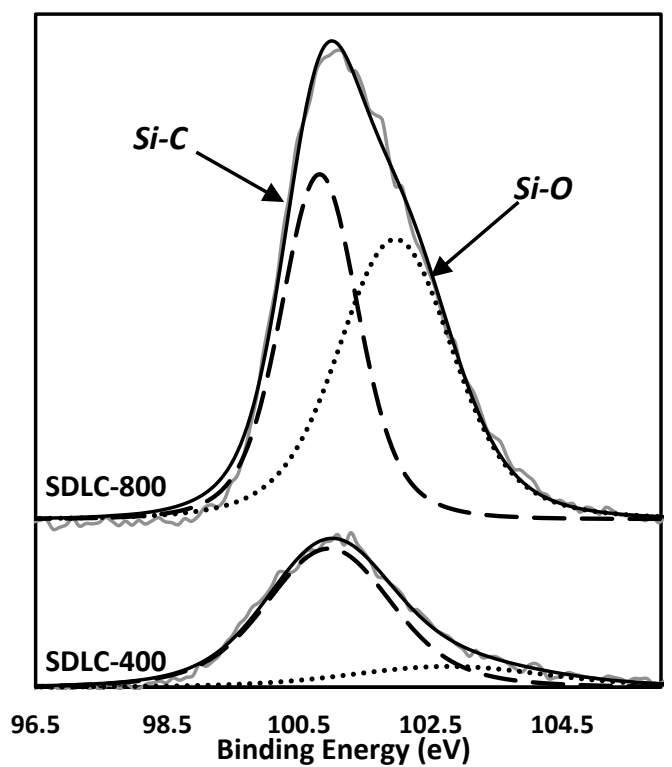

Figure 4.23. Si2p XPS spectra of Silicon doped DLC samples with different Si concentrations

Comparing peak positions in TEY spectra of DLC with those of N-DLC samples, upshift of binding energy was observed, which is probably due to the formation of $\mathrm{C}-\mathrm{N} \mathrm{sp}^{2}$ and $\mathrm{sp}^{3}$ bonding. Nevertheless, TEY spectra show stronger $\mathrm{sp}^{3}$ peak at $292 \mathrm{eV}$ compared to FLY spectra, which could be due to higher $\mathrm{C}-\mathrm{H} \mathrm{sp}{ }^{3}$ content on the surface. There are two main peaks around $285 \mathrm{eV}$ and $292 \mathrm{eV}$ in the FLY spectra displayed in Figure 4.25, consistent with a previous report [100]. The peaks around $288 \mathrm{eV}$ and $287.5 \mathrm{eV}$ disappear and a new small peak appears around 286.5 in samples with relatively higher nitrogen concentrations (NDLC-2 and NDLC-3). This implies that the bulk of the coating is mostly consist of $\mathrm{sp}^{2} \mathrm{C}=\mathrm{C}$ and $\mathrm{sp}^{3} \mathrm{C}-\mathrm{C}$ bonds. Furthermore, NDLC-1 and DLC-M show similar spectra, demonstrating small amount of nitrogen doped into DLC has little effect on carbon bonding states. 


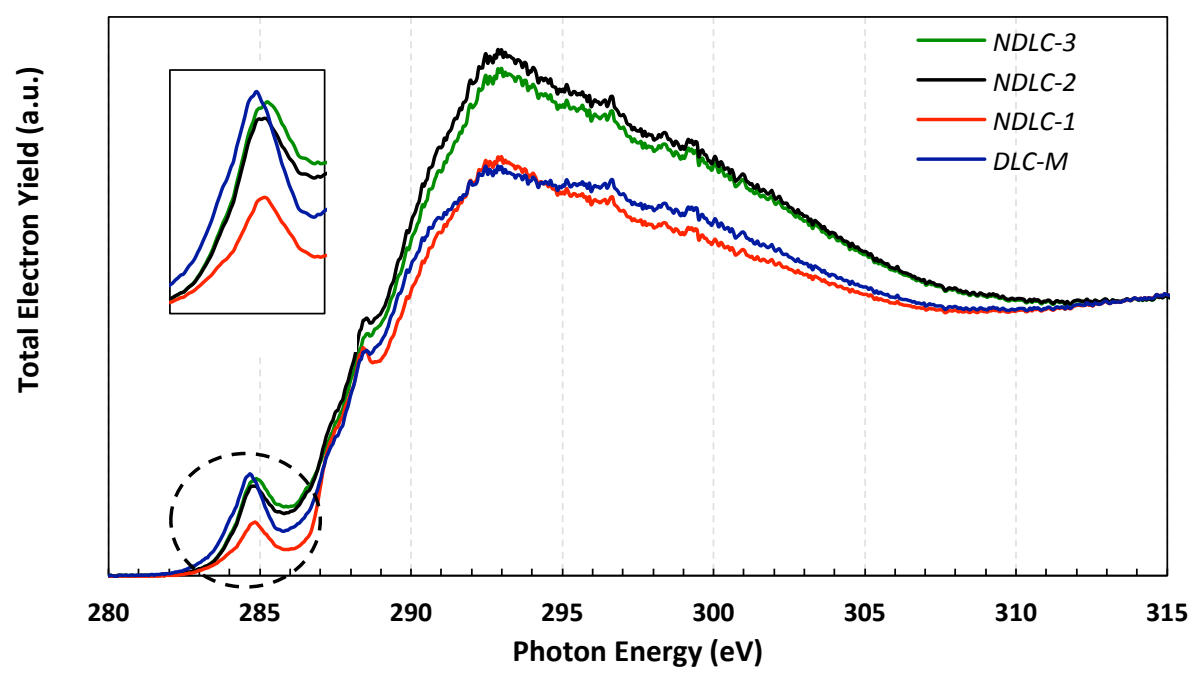

Figure 4.24. NEXAFS spectra of N-DLC and DLC in TEY mode

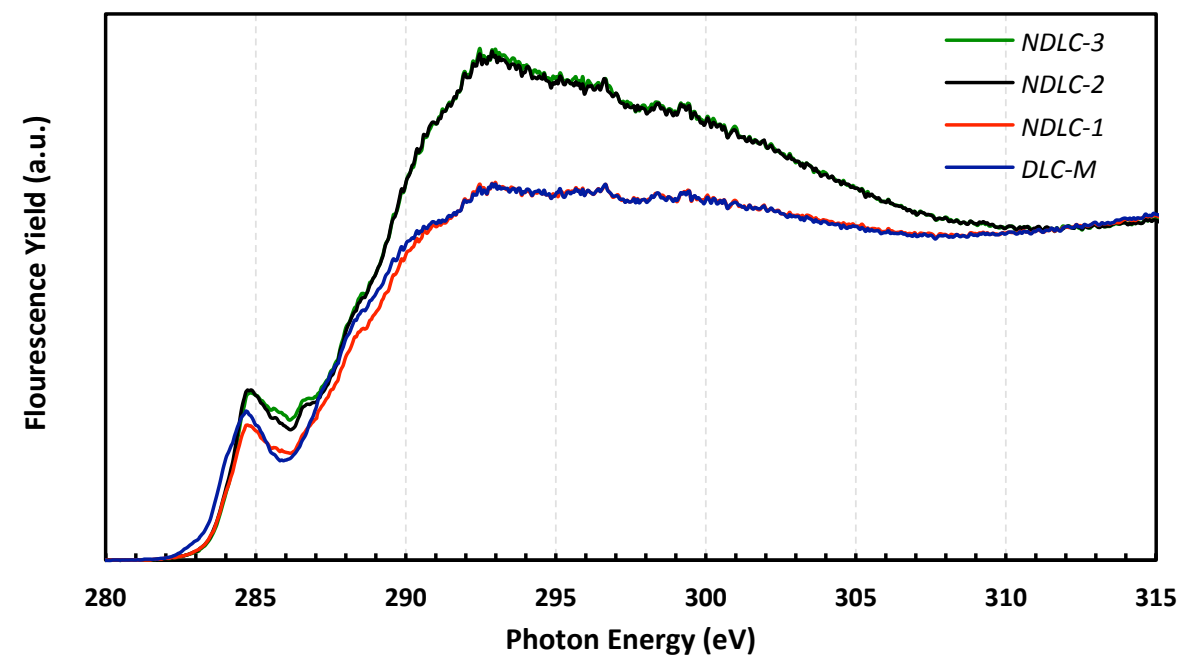

Figure 4.25. NEXAFS spectra of N-DLC and DLC in FLY mode

\subsubsection{Mechanical properties}

The hardness and Young's modulus of N-DLC and Si-DLC is shown in Figure 4.26, which were obtained with nano-indentation testing on nine different locations for each sample. The hardness and Young's modulus of DLC-M was around $11.5 \mathrm{GPa}$ and $116 \mathrm{GPa}$, respectively. As it can be found in Figure 4.26, the hardness of film drops with increasing nitrogen concentration while the Young's modulus decreases at lower $\mathrm{N}$ concentration and rise by increasing nitrogen content. For silicon doped DLC, both hardness and Young's modulus of the films decrease at low concentrations and increase by incorporation of more silicon into coating. The hardness of DLC is strongly dependent on the amount of $\mathrm{sp}^{3} \mathrm{C}-\mathrm{C}$ boning within it. Incorporation of nitrogen into 
coating results in $\mathrm{C}=\mathrm{N}$ bonding and consequently $\mathrm{sp}^{3}$ concentration declines. Thus more graphitic coating with lower internal stress would formed with nitrogen doping, which results in lower film hardness, as reported by other researchers [17]. In silicon doped samples, $\mathrm{sp}^{3} \mathrm{Si}-\mathrm{C}$ bonds form that usually reduced intrinsic stress in the films. Reduced stress decreases hardness while increased $\mathrm{sp}^{3}$ bonds increases hardness. Reduced hardness at low silicon concentration would be mainly due to the reduced stress while higher silicon concentration results in higher $\mathrm{sp}^{3}$ bonds and would increase hardness [19].
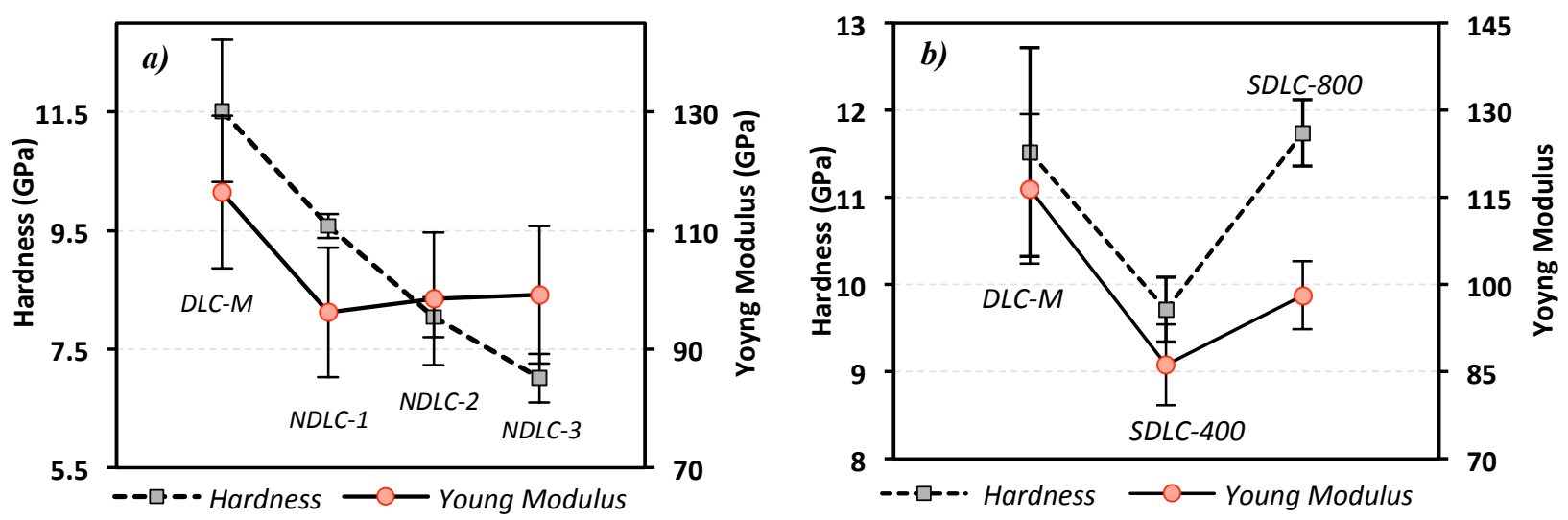

Figure 4.26. Hardness and young modulus of a) Nitrogen doped DLC, b) Silicon doped DLC with different concentrations

\subsubsection{Scratching results}

Figure 4.27 illustrates optical microscopic results obtained from samples NDLC-3 and SDLC800 after scratch testing under an increasing load from 0.1 to $0.5 \mathrm{mN}$, in the same position (the middle of the line). As it can be seen, no delamination was observed for N-DLC samples while detachment of films was observed around the scratch for Si-DLC, indicating higher adhesion of N-DLC compared to S-DLC. Formation of high amount of graphitic bonds has been confirmed by the results of XPS and Raman spectra of N-DLC samples. Because of the higher $\mathrm{sp}^{2}$ concentration, N-DLC films show lower internal stress and thus stronger adhesion to PET substrate. In addition, the nitrogen doped could interact with PET and play a role in improving the film adhesion. On the contrary, Si-DLC samples show increased $\mathrm{sp}^{3}$ concentration and thus higher internal stress, which decreases the adhesion strength of film to substrate. 

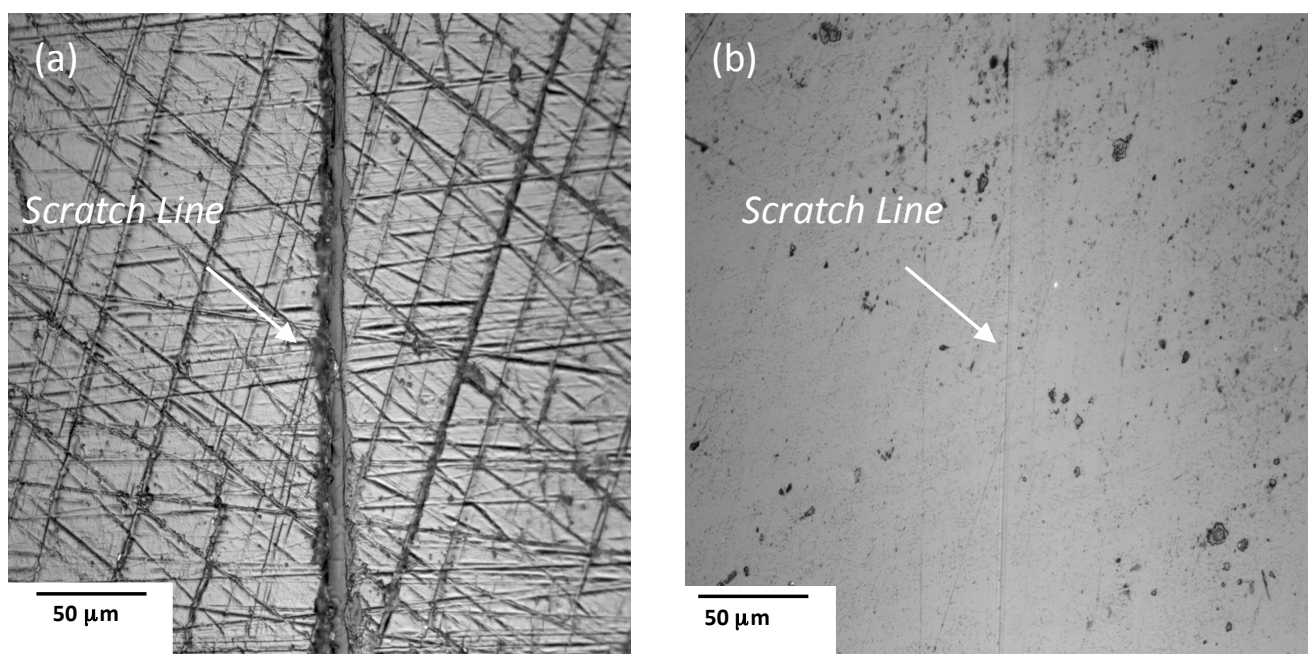

Figure 4.27. Optical microscopy of a) SDLC-800 and b) NDLC-3 after scratch testing

\subsubsection{Friction and wear}

It has been noted that the tribological behavior of DLC is highly dependent on the testing environment as well as the structure of DLC. The stable COF for different samples is presented in Figure 4.28. It shows that by incorporation of nitrogen into DLC, COF initially increased to a higher value for NDLC-1 compared to pure DLC while when further increasing nitrogen content, the COF decreases, in agreement with previous reported results $[112,113]$. The decrease of COF with increasing $\mathrm{N}$ content is probably due to the decreased surface roughness shown in Figure 4.28. Nevertheless, COF of silicon-doped samples is much higher than bare DLC, which is contradictory to the previous reported results [56]. The increase in COF in this study could be due to high surface roughness and formation of $\mathrm{sp}^{3} \mathrm{Si}-\mathrm{C}$ bonding [114]. However, by increasing the silicon content, $\mathrm{COF}$ decreases, which could be due to formation of $\mathrm{SiO}_{2}$ on the surface [114]. 


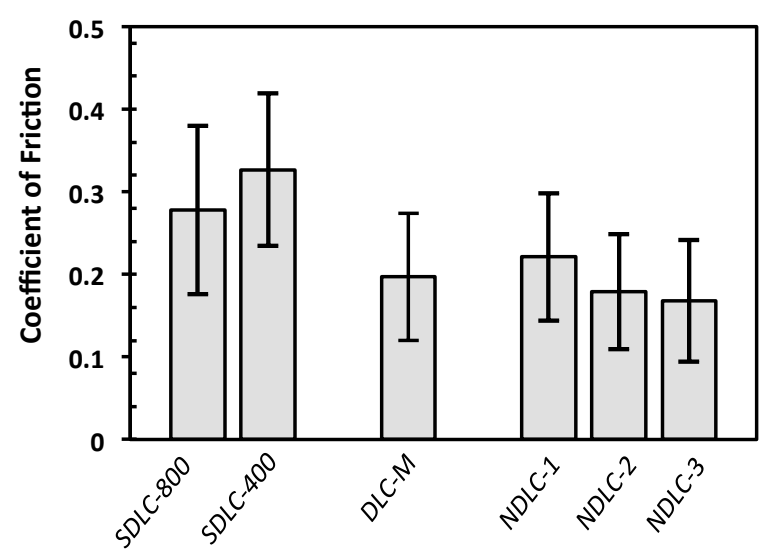

Figure 4.28. COF of DLC, N-DLC and Si-DLC samples under reciprocating motion

\subsubsection{Contact angle and surface energy}

The contact angle of bare PET utilized in our study is $81.2^{\circ}$ and the calculated surface energy is $83.9 \mathrm{mN} / \mathrm{m}$. The contact angle of DLC coated PET is $71.3^{\circ}$ and the calculated surface energy is $96.2 \mathrm{mN} / \mathrm{m}$. Nitrogen doping increases contact angle to $72.5^{\circ}, 75^{\circ}$, and $75.3^{\circ}$ for N-DLC-1, NDLC-2, and NDLC-3, respectively. On the other hand, silicon doping increases the contact angle and thus decreases the surface energy to be $79 \mathrm{mN} / \mathrm{m}$ for SDLC-800. The change in surface energy is closely related to the variation of carbon bonds $\left(\mathrm{sp}^{3} / \mathrm{sp}^{2}\right.$ ratio), hydrogen content on the film surface, and the surface roughness caused by doping [86].

Table 4.6. Roughness, water contact angle and surface energy values for different DLC samples

\begin{tabular}{|c|c|c|c|c|}
\hline & $\begin{array}{c}\text { Sample } \\
\text { name }\end{array}$ & $\begin{array}{c}\text { Roughness } \\
\text { (nm) }\end{array}$ & $\begin{array}{l}\text { Contact } \\
\text { angle }\left({ }^{\circ}\right)\end{array}$ & $\begin{array}{c}\text { Surface } \\
\text { Energy } \\
(m N / m)\end{array}$ \\
\hline$a$ & Bare PET & $13 \pm 1$ & $81.2 \pm 0.1$ & $83.9 \pm 0.1$ \\
\hline $\boldsymbol{b}$ & $D L C-M$ & $143 \pm 1$ & $71.3 \pm 0.1$ & $96.2 \pm 0.1$ \\
\hline$c$ & $N D L C-1$ & $29 \pm 1$ & $72.5 \pm 0.1$ & $95.2 \pm 0.1$ \\
\hline$d$ & $N D L C-2$ & $17 \pm 1$ & $74.9 \pm 0.1$ & $91.8 \pm 0.1$ \\
\hline$e$ & $N D L C-3$ & $14 \pm 1$ & $75.3 \pm 0.1$ & $91.23 \pm 0.1$ \\
\hline$f$ & $S D L C-400$ & $81 \pm 1$ & $77.0 \pm 0.1$ & $89.2 \pm 0.1$ \\
\hline$h$ & $S D L C-800$ & $91 \pm 1$ & $84.9 \pm 0.2$ & $79.3 \pm 0.2$ \\
\hline
\end{tabular}




\subsubsection{Surface roughness}

Table 4.6 summarized the roughness values for bare PET, pure DLC, N-SLC and Si-DLC samples measured by the optical profiler. The surface roughness of PET before deposition is 12.9 $\mathrm{nm}$ and increases dramatically after DLC deposition. Pure DLC has the highest roughness. Both nitrogen and silicon doping reduces the roughness and the higher the nitrogen content, the lower the roughness but the higher the Si content, the higher the roughness. The high roughness of DLC coated samples is probably caused by the micorcracks induced by the high stress in the film as it can be seen in Figure 4.29. Nitrogen incorporation could decrease coating internal stress by formation of $\mathrm{sp}^{2}$ graphitic bonds and replacement of $\mathrm{C}-\mathrm{C}$ bonds with $\mathrm{C}-\mathrm{N}$ bonds while silicon doping reduces stress and facilitates $\mathrm{sp}^{3}$ bonds. The reduced stress in N-DLC and Si-DLC decrease the probability to form micarocracks and thus decreases roughness. These results are in agreement with the hardness measurement results, the harder the coating, the higher the roughness. 


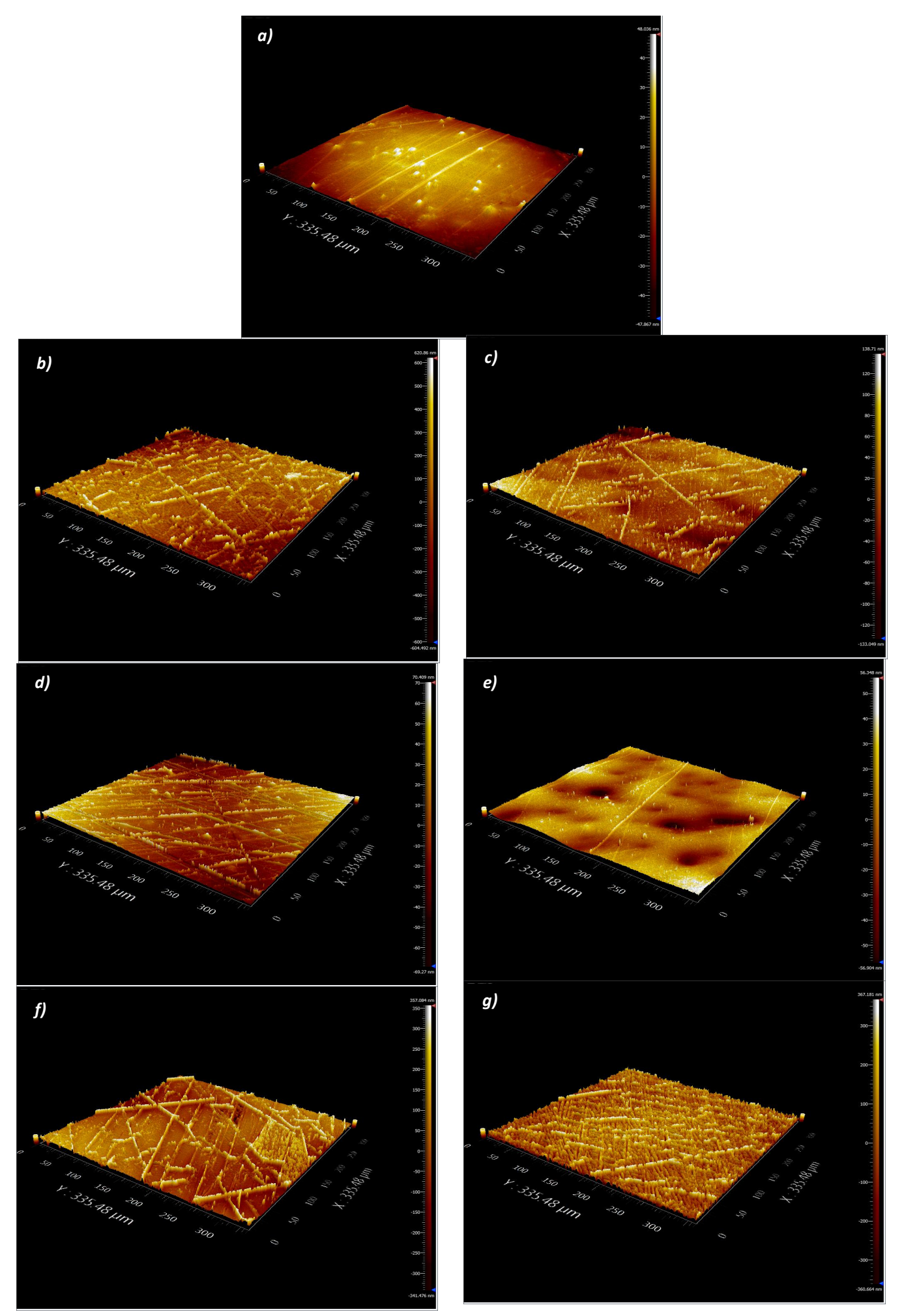

Figure 4.29. Roughness measurement with optical profiler of a) bare PET, b) DLC-M, c) NDLC1, d) NDLC-2, d) NDLC-3, f) SDLC-400, g) SDLC-800 


\section{CHAPTER 5}

\section{SUMMARY, CONCLUSIONS, AND FUTURE WORK}

Hydrogenated diamond like carbon thin films with different compositions (DLC, N-DLC and SiDLC) have been deposited on PET films and silicon wafers by using end-hall ion beams with relatively low energy and characterized using various advanced technologies. In addition, the effects of ion surface treatment in oxygen, nitrogen and argon plasma environments on adhesion enhancement of DLC on polymer have been investigated. The results and conclusions are summarized as follows:

\subsection{Summary}

- Oxygen ion treatment can effectively changes the chemical states of PET and improve the adhesion of DLC significantly and the increase of treatment time can further improve DLC adhesion.

- DLC films deposited with methane as precursor gas show superior mechanical and adhesive properties than those films deposited with acetylene.

- Si doping increases $\mathrm{sp}^{3}$ carbon bonding content in the films while $\mathrm{N}$ doping decreases $\mathrm{sp}^{3}$ carbon bonding content in the films. The doped $\mathrm{Si}$ forms $\mathrm{sp}^{3} \mathrm{Si}-\mathrm{C}$ bonds and $\mathrm{N}$ forms $\mathrm{sp}^{3}$ $\mathrm{C}-\mathrm{N}$ and $\mathrm{sp}^{2} \mathrm{C}=\mathrm{N}$ bonds in the films. Consequently, the hardness values decrease from 11.5 GPa for pure DLC to 7.0 GPa for N-DLC samples whereas the hardness of Si-DLC increases with increasing silicon content.

- Nitrogen doping promotes formation of $\mathrm{sp}^{2}$ configuration due to the formation of $\mathrm{sp}^{2}$ $\mathrm{C}=\mathrm{N}$ bonds and thus reduces surface roughness and internal stress, consequently improves film adhesion to PET while Si doping forms $\mathrm{sp}^{3} \mathrm{Si}-\mathrm{C}$ and thus increases hardness, Young's modulus, COF, and roughness. 


\subsection{Conclusions}

- Ion treatment can effectively change the microstructure and chemical states of PET polymer. The kind of ions and the treatment time play an important role in PET structure changes. The enhancement of adhesion is due to formation of free radicals and new chemical bonds on the treated surface.

- The DLC films deposited using methane show better properties than using acetylene.

- Element doping can change the structure of DLC and thus the mechanical properties. In addition, $\mathrm{N}$ doping can reduce residual stress of DLC and thus increases adhesion of DLC. By optimizing the conditions, dense, smooth, and adhesive DLC films have been deposited on PET using EH ion source by combining $\mathrm{N}$ doping and $\mathrm{O}$ ion treatment with methane as precursor gas.

- Nitrogen doped DLC films could provide effective mechanical and wear protection to PET and is very promising for many relevant applications including food and beverage containers and biomedical implants.

\subsection{Future work}

Dense smooth adhesive DLC films have been successfully deposited onto PET using EH ion beam source. As reviewed, coated PET is very promising to be used as food and beverage containers and biomedical implants. Therefore, investigation of the gas barrier properties and the biocompatibility of the N-DLC coated PET is desirable and interesting in the future. 


\section{APPENDIX}

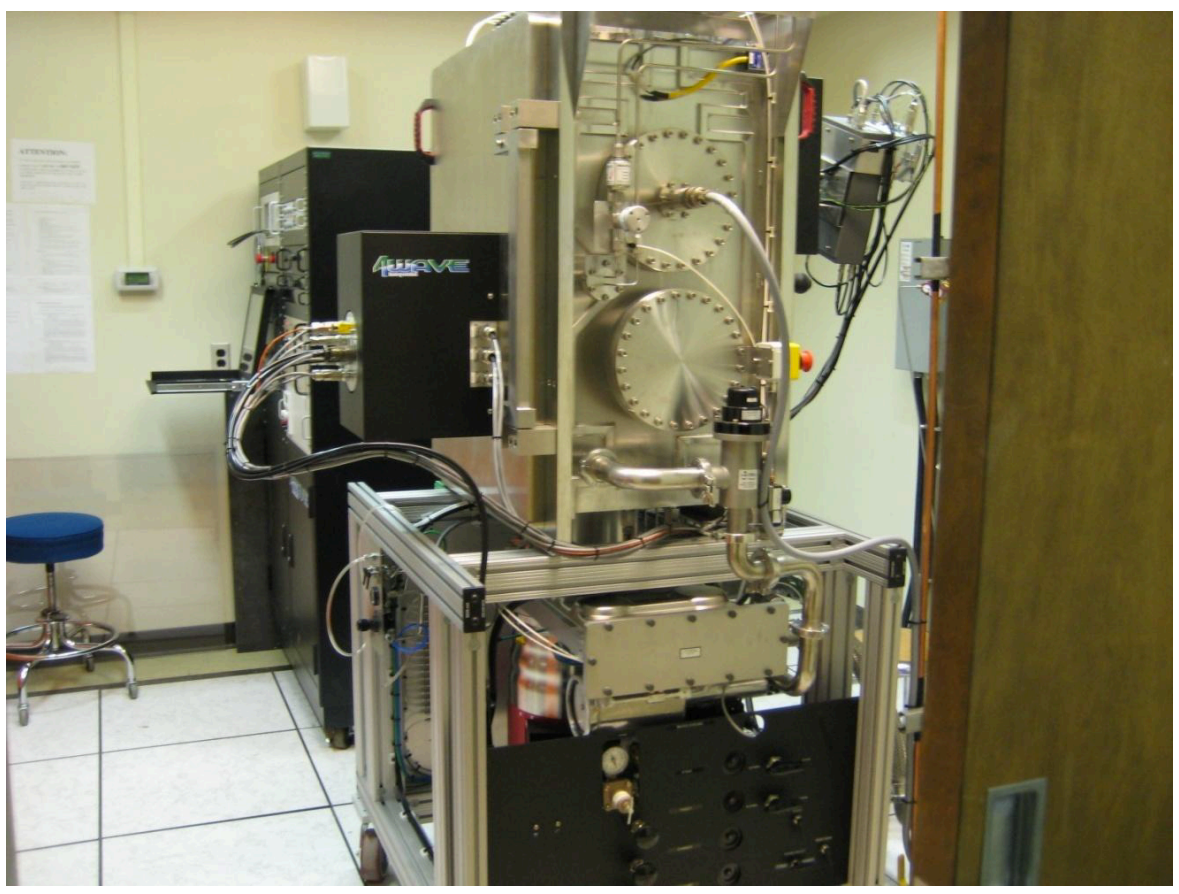

Figure A.1. Picture of IBD system

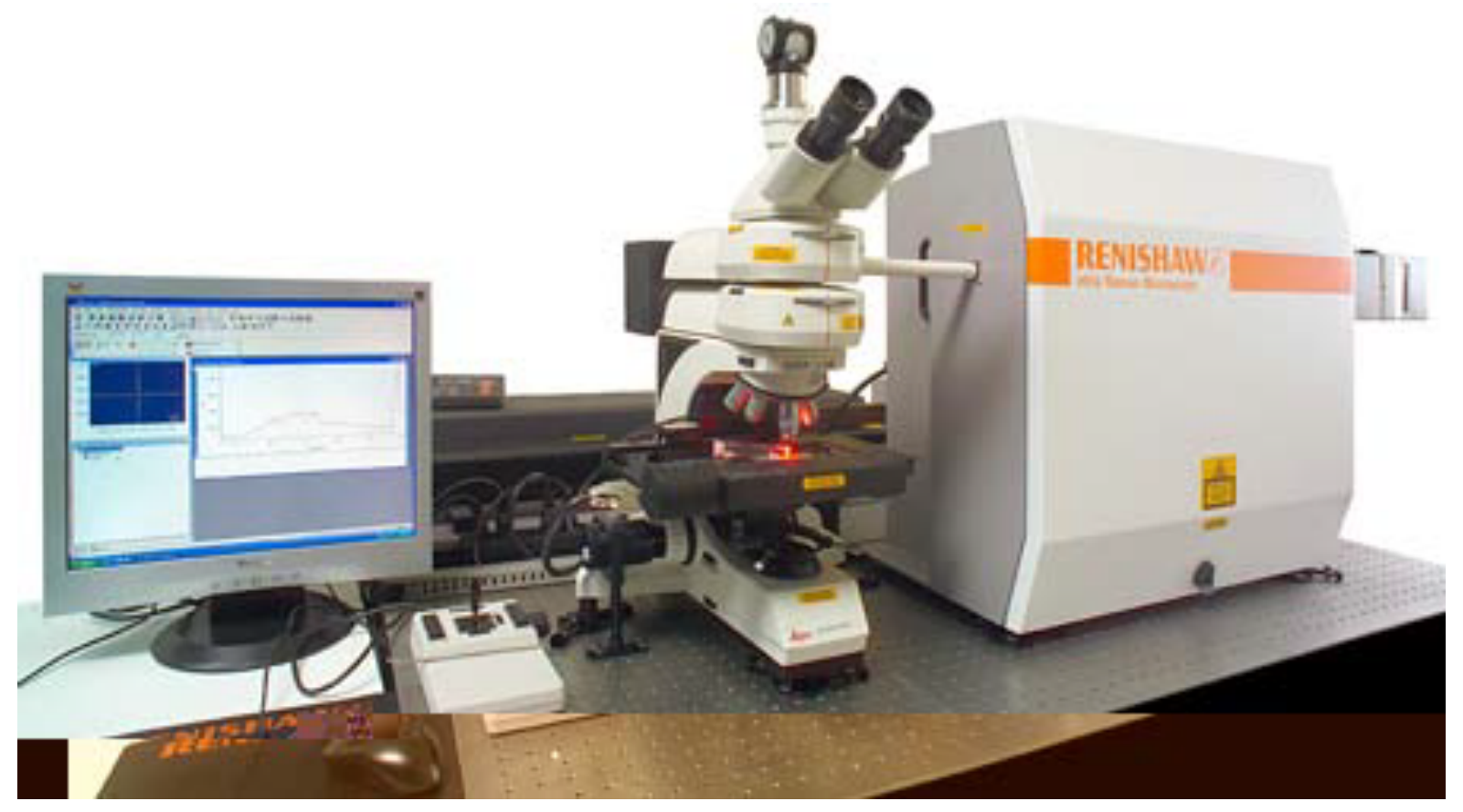

Figure A.2. A picture of Renishaw 2000 micro-Raman system 


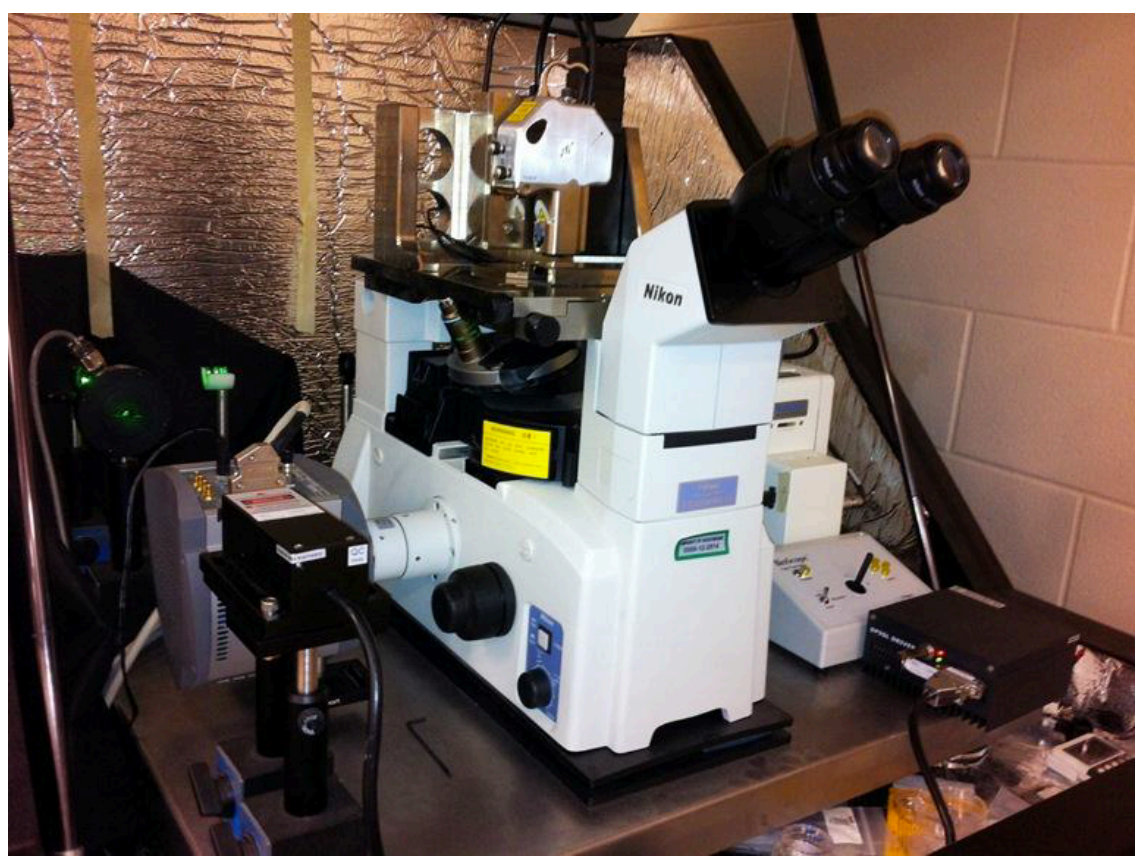

Figure A.3. Picture of AFM mounted with an optical microscope

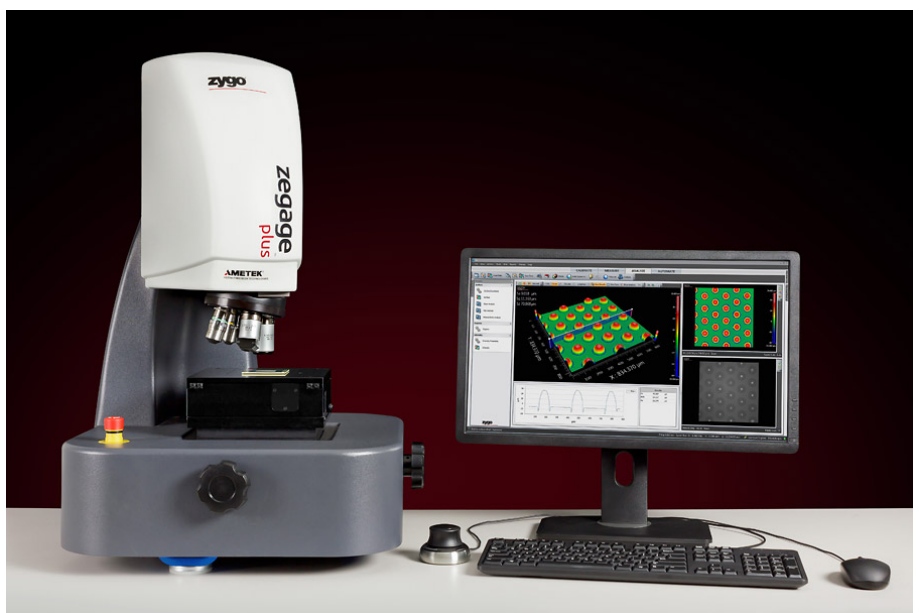

Figure A.4. Optical profilometer (http://www.zygo.com/met/profilers/zegageplus/zegage-plusoptical-profiler_ws_lg.jpg 


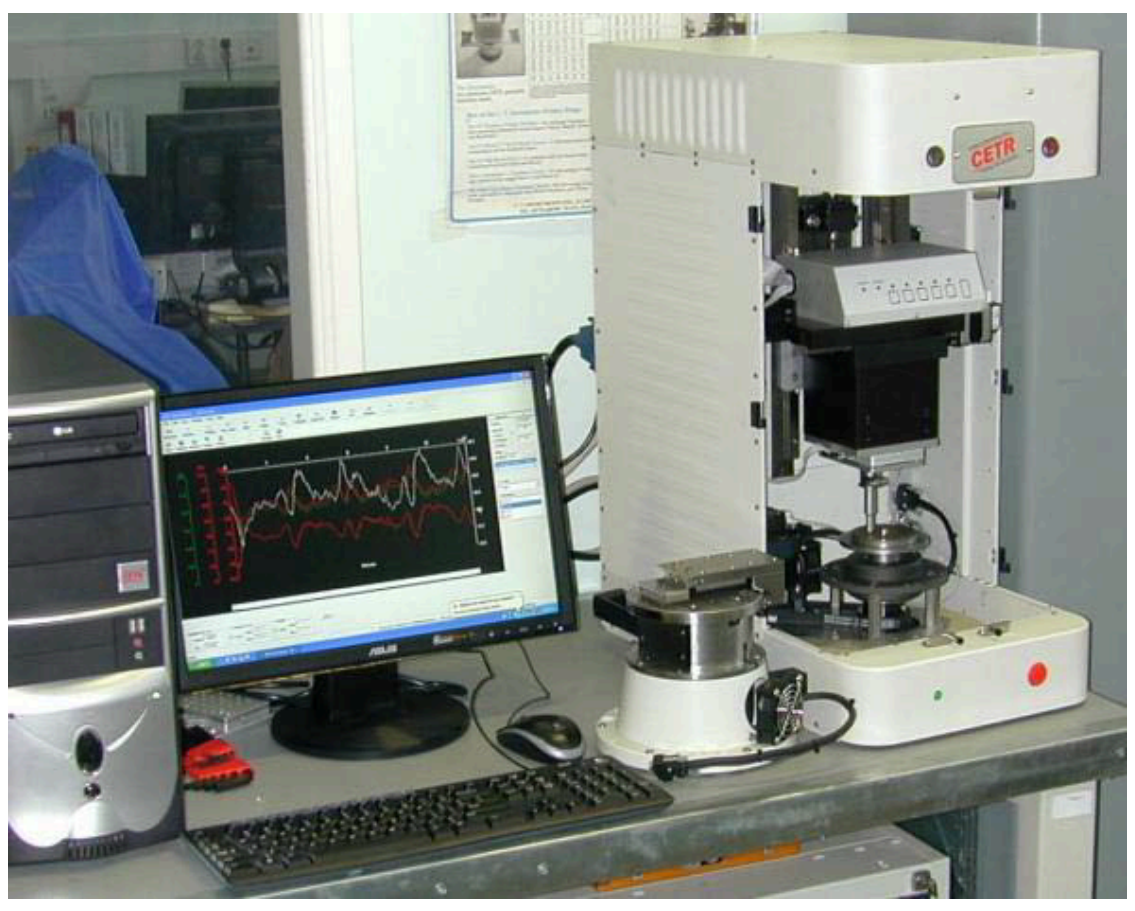

Figure A.5. Picture of Universal Material Tester designed and made by CETR

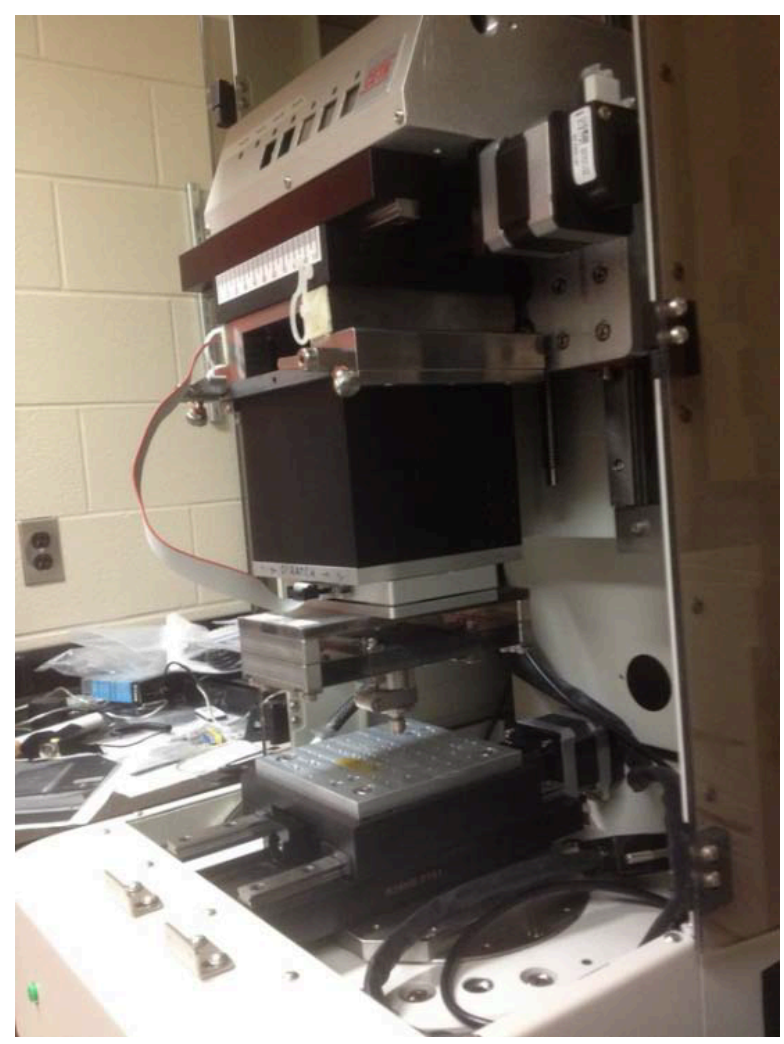

Figure A.6. UMT during the ball-on-disk testing 


\section{REFERENCES}

[1] K.N. Pandiyaraj, J. Heeg, A. Lampka, F. Junge, T. Barfels, M. Wienecke, et al., Cyto and Blood Compatibility of Titanium Containing Diamond-Like Carbon Prepared by Hybrid Sputtering Method, Plasma Sci. Technol. 14 (2012) 829-836.

[2] A. Ogino, M. Nagatsu, Gas barrier properties of hydrogenated amorphous carbon films coated on polymers by surface-wave plasma chemical vapor deposition, Thin Solid Films. 515 (2007) 3597-3601.

[3] H. Tashiro, M. Nakaya, A. Hotta, Enhancement of the gas barrier property of polymers by DLC coating with organosilane interlayer, Diam. Relat. Mater. 35 (2013) 7-13.

[4] S. Venkatachalam, S. Nayak, J. Labde, Degradation and Recyclability of Poly (Ethylene Terephthalate), Polyester. (2012) 75-98.

[5] I.E.R. Series, PACK, Polyethylene Terephtalate (PET) for food packaging applications, 2000 .

[6] J. Shim, H. Gyu, S. Na, I. Kim, S. Kwak, Silicon oxynitride gas barrier coatings on poly ( ether sulfone ) by plasma-enhanced chemical vapor deposition, Surf. Coat. Technol. 202 (2008) 2844-2849.

[7] S. Kim, M. Haque, W. Kim, G. Kim, Effects of argon and oxygen $\mathrm{fl}$ ow rate on water vapor barrier properties of silicon oxide coatings deposited on polyethylene terephthalate by plasma enhanced chemical vapor deposition, Thin Solid Films. 518 (2010) 1929-1934.

[8] J.M. Lackner, C. Meindl, C. Wolf, A. Fian, C. Kittinger, M. Kot, et al., Gas Permeation, Mechanical Behavior and Cytocompatibility of Ultrathin Pure and Doped Diamond-Like Carbon and Silicon Oxide Films, Coatings. (2013) 268-300.

[9] R. Hauert, A review of modified DLC coatings for biological applications, Diam. Relat. Mater. 12 (2003) 583-589.

[10] S. V. Hainsworth, N.J. Uhure, Diamond like carbon coatings for tribology: production techniques, characterisation methods and applications, Int. Mater. Rev. 52 (2007) 153175.

[11] A. Shirakura, M. Nakaya, Y. Koga, H. Kodama, T. Hasebe, T. Suzuki, Diamond-like carbon films for PET bottles and medical applications, Thin Solid Films. 494 (2006) 8491.

[12] D. Tsubone, H. Kodama, T. Hasebe, A. Hotta, Gas barrier properties and periodically fractured surface of thin DLC films coated on flexible polymer substrates, Surf. Coatings Technol. 201 (2007) 6431-6436.

[13] E.M. Liston, L. Martinu, M.R. Wertheimer, Plasma surface modification of polymers for improved adhesion: a critical review, J. Adhes. Sci. Technol. 7 (1993) 1091-1127.

[14] M.G. Katsikogianni, C.S. Syndrevelis, E.K. Amanatides, D.S. Mataras, Y.F. Missirlis, Plasma treated and a-C:H coated PET performance in inhibiting bacterial adhesion, Plasma Process. Polym. 4 (2007) 1046-1051.

[15] J.C. Sánchez-López, A. Fernández, Doping and alloying effects on DLC coatings, Tribol. Diamond-Like Carbon Film. Fundam. Appl. (2008) 311-328. 
[16] J. Robertson, Diamond-like amorphous carbon, Mater. Sci. Eng. R Reports. 37 (2002) 129-281.

[17] S. Bhattacherjee, H. Niakan, Q. Yang, Y. Hu, J. Dynes, Enhancement of adhesion and corrosion resistance of diamond-like carbon thin fi lms on $\mathrm{Ti}-6 \mathrm{Al}-4 \mathrm{~V}$ alloy by nitrogen doping and incorporation of nanodiamond particles, Surf. Coat. Technol. 284 (2015) 153158.

[18] V. Anita, T. Butuda, T. Maeda, K. Takizawa, N. Saito, O. Takai, Effect of N doping on properties of diamond-like carbon thin films produced by RF capacitively coupled chemical vapor deposition from different precursors, Diam. Relat. Mater. 13. 13 (2004) 1993-1996.

[19] G. a. Abbas, J. a. McLaughlin, E. Harkin-Jones, A study of ta-C, a-C:H and Si-a:C:H thin films on polymer substrates as a gas barrier, Diam. Relat. Mater. 13 (2004) 1342-1345.

[20] H.R. Kaufman, End-Hall ion source, J. Vac. Sci. Technol. A Vacuum, Surfaces, Film. 5 (1987) 2081.

[21] Y. Tang, Y.S. Li, Q. Yang, a. Hirose, Characterization of hydrogenated amorphous carbon thin films by end-Hall ion beam deposition, Appl. Surf. Sci. 257 (2011) 46994705 .

[22] H. Niederwald, S. Laux, M. Kennedy, U. Schallenberg, A. Duparre', M. Mertin, et al., Ion-assisted deposition of oxide materials Characterization of hydrogenated amorphous carbon thin films by end-Hall ion beam deposition, Appl. Opt. 38 (1999) 3610-3613.

[23] S. Esmaeili Khatir, Nitrogen-doped dlC deposition by hot filament and inductively coupled plasma sputtering for biomedical applications, MSc Thesis in Department of Physics and Engineering Physics, University of Saskatchewan, 2013.

[24] J. Robertson, Amorphous carbon, Curr. Opin. Solid State Mater. Sci. 1 (1996) 557-561.

[25] K. Singh, O. Anil, S.K. Dhawan, Polymer-Graphene Nanocomposites: Preparation , Characterization, Properties, and Applications(pp. 37-72). InTech, Hampshire, 2012.

[26] J.C. Angus, Dense "“diamondlike"” hydrocarbons as random covalent networks, J. Vac. Sci. Technol. A Vacuum, Surfaces, Film. 6 (1988) 1778.

[27] J. Robertson, Diamond-like amorphous carbon, 37 (2002) 129-281.

[28] A. Erdemir, C. Donnet, Tribology of diamond-like carbon films: recent progress and future prospects, J. Phys. D Appl. Phys. 39 (2006) 311-327.

[29] O. Monteiro, Synthesis, properties and applications of pure and covalently doped DLC films prepared by energetic condensation, Lawrence Berkeley Natl. Lab. (2001).

[30] A. Erdemir, C. Donnet, Tribology of Diamond, Diamond-Like Carbon, and Related Films, University of Chicago as Operator of Argonne National Laboratory ("Argonne"), 2001.

[31] H. Nakazawa, A. Sudoh, M. Suemitsu, K. Yasui, T. Itoh, T. Endoh, et al., Mechanical and tribological properties of boron, nitrogen-coincorporated diamond-like carbon films prepared by reactive radio-frequency magnetron sputtering, Diam. Relat. Mater. 19 (2010) 503-506.

[32] O.R. Monteiro, Synthesis, Properties and Applications of Pure and Covalently Doped 
DLC Films Prepared by Energetic Condensation, Berkeley Lab. (2001) https://escholarship.org/uc/item/9vx2p89j.

[33] Y. Lifshitz, Diamond-like carbon — present status, Diam. Relat. Mater. 8 (1999) 16591676.

[34] X. He, Diamond-like carbon film synthesized by ion beam assisted deposition and its tribological properties, J. Vac. Sci. Technol. A Vacuum, Surfaces, Film. 14 (1996) 2039.

[35] J. Robertson, The deposition mechanism of diamond-like a-C and a-C: H, Diam. Relat. Mater. 3 (1994) 361-368.

[36] D.S. Finch, J. Franks, N.X. Randall, a Barnetson, J. Crouch, a C. Evans, et al., Diamond-like carbon, a barrier coating for polymers used in packaging applications, Packag. Technol. Sci. 9 (1996) 73-85.

[37] H. Kodama, A. Shirakura, A. Hotta, T. Suzuki, Gas barrier properties of carbon films synthesized by atmospheric pressure glow plasma, Surf. Coatings Technol. 201 (2006) 913-917.

[38] G.A. Abbas, S.S. Roy, P. Papakonstantinou, J.A. McLaughlin, Structural investigation and gas barrier performance of diamond-like carbon based films on polymer substrates, Carbon N. Y. 43 (2005) 303-309.

[39] Y. Tang, Y.S. Li, C.Z. Zhang, J. Wang, Q. Yang, A. Hirose, Synthesis of cobalt/diamondlike carbon thin films by biased target ion beam deposition, Diam. Relat. Mater. 20 (2011) 538-541.

[40] P.V. Bharathy, D. Nataraj, D. Mangalaraj, M.S.R.N. Kiran, J. Silvestre-Albero, Q. Yang, Influence of Tungsten Content in W-Dlc Nanocomposite Thin Films Prepared By Hybrid Target Biased Ion Beam Assisted Deposition Technique, Int. J. Nanosci. 10 (2011) 851855.

[41] B. Podgornik, D. Hren, J. Vižintin, Low-friction behaviour of boundary-lubricated diamond-like carbon coatings containing tungsten, Thin Solid Films. 476 (2005) 92-100.

[42] D.Y. Wang, K.W. Weng, S.Y. Hwang, Study on metal-doped diamond-like carbon films synthesized by cathodic arc evaporation, Diam. Relat. Mater. 9 (2000) 1762-1766.

[43] C. Liu, W. Gou, G. Li, L. Wang, Z. Mu, Improvement of mechanical properties of diamond-like carbon film by Ti ion implantation, Plasma Process. Polym. 4 (2007) 265268.

[44] R.K.Y. Fu, Y.F. Mei, M.Y. Fu, X.Y. Liu, P.K. Chu, Thermal stability of metal-doped diamond-like carbon fabricated by dual plasma deposition, Diam. Relat. Mater. 14 (2005) 1489-1493.

[45] C. Bauer, H. Leiste, M. Stüber, S. Ulrich, H. Holleck, M. Stuber, et al., Mechanical properties and performance of magnetron-sputtered graded diamond-like carbon films with and without metal additions, Diam. Relat. Mater. 11 (2002) 1139-1142.

[46] V. Singh, V. Palshin, R.C. Tittsworth, E.I. Meletis, Local structure of composite Crcontaining diamond-like carbon thin films, Carbon N. Y. 44 (2006) 1280-1286.

[47] G. Ma, S. Gong, G. Lin, L. Zhang, G. Sun, A study of structure and properties of Tidoped DLC film by reactive magnetron sputtering with ion implantation, Appl. Surf. Sci. 
258 (2012) 3045-3050.

[48] K. Ma, G. Yang, L. Yu, P. Zhang, Synthesis and characterization of nickel-doped diamond-like carbon film electrodeposited at a low voltage, Surf. Coatings Technol. 204 (2010) 2546-2550.

[49] M.J. Dai, K.S. Zhou, S.S. Lin, H.J. Hou, X.G. Zhu, H.W. Li, et al., A study on metaldoped diamond-like carbon film synthesized by ion source and sputtering technique, Plasma Process. Polym. 4 (2007) 215-219.

[50] S. Srinivasan, Y. Tang, Y.S. Li, Q. Yang, a. Hirose, Ion beam deposition of DLC and nitrogen doped DLC thin films for enhanced haemocompatibility on PTFE, Appl. Surf. Sci. 258 (2012) 8094-8099.

[51] W. Kautek, S. Pentzien, A. Conradi, J. Kriiger, K. Brzezinka, Pulsed-laser deposition and boron-blending of diamond-like carbon ( DLC ) thin films, Appl. Surf. Sci. 106 (1996) $158-165$.

[52] M. Kahn, N. Menegazzo, B. Mizaikoff, R. Berghauser, J.M. Lackner, D. Hufnagel, et al., Properties of DLC and nitrogen-doped DLC films deposited by DC magnetron sputtering, Plasma Process. Polym. 4 (2007) 200-204.

[53] Z.Q. Yao, P. Yang, N. Huang, H. Sun, J. Wang, Structural, mechanical and hydrophobic properties of fluorine-doped diamond-like carbon films synthesized by plasma immersion ion implantation and deposition (PIII-D), Appl. Surf. Sci. 230 (2004) 172-178.

[54] T. Hasebe, S. Nagashima, A. Kamijo, T. Yoshimura, T. Ishimaru, Y. Yoshimoto, et al., Depth profiling of fluorine-doped diamond-like carbon (F-DLC) film: Localized fluorine in the top-most thin layer can enhance the non-thrombogenic properties of F-DLC, Thin Solid Films. 516 (2007) 299-303.

[55] C. Donnet, Recent progress on the tribology of doped diamond-like and carbon alloy coatings: a review, Surf. Coatings Technol. 100-101 (1998) 180-186.

[56] W.-J. Wu, T.-M. Pai, M.-H. Hon, Wear behavior of silicon-containing diamond-like carbon coatings, Diam. Relat. Mater. 7 (1998) 1478-1484.

[57] T. Hasebe, A. Hotta, H. Kodama, A. Kamijo, Recent Advances in Diamond-Like Carbon Films in the Medical and Food Packing Fields, New Diam. Front. Carbon Technol. 17 (2008) 2007.

[58] L. Zhang, Synthesis and Characterization of Boron Incorporated Diamond-Like Carbon Thin Films, MSc thesis in Department of Mechanical Engineering, University of Saskatchewan, (2012).

[59] N. Hellgren, M.P. Johansson, E. Broitman, L. Hultman, J.. Sundgren, Role of nitrogen in the formation of hard and elastic $\mathrm{CNx}$ thin films by reactive magnetron sputtering, Phys. Rev. B. 59 (1999) 5162-5177.

[60] Y. Kusano, J.E. Evetts, R.E. Somekh, I.M. Hutchings, Properties of carbon nitride deposited by magnetron sputtering, Thin Solid Films. 332 (1998) 56-61.

[61] a. Grill, Tribology of diamondlike carbon and related materials: an updated review, Surf. Coatings Technol. 94-95 (1997) 507-513.

[62] Y.S. Zou, Q.M. Wang, H. Du, G.H. Song, J.Q. Xiao, J. Gong, et al., Structural 
characterization of nitrogen doped diamond-like carbon films deposited by arc ion plating, Appl. Surf. Sci. 241 (2005) 295-302.

[63] D.A. Anderson, W.E. Spear, Electrical and optical properties of amorphous silicon carbide, silicon nitride and germanium carbide prepared by the glow discharge technique, Philos. Mag. 35 (1977) 1-16.

[64] A. Morimoto, T. Miura, M. Kumeda, T. Shimizu, Defects in hydrogenated amorphous silicon-carbon alloy films prepared by glow discharge decomposition and sputtering, J. Appl. Phys. 53 (1982) 7299-7305.

[65] D.R. McKenzie, N. Savvides, D.R. Mills, R.C. McPhedran, L.C. Botten, Optical constants of amorphous hydrogenated carbon and silicon-carbon alloy films and their application in high temperature solar selective surfaces, Sol. Energy Mater. 9 (1983) 113126.

[66] K. Oguri, T. Arai, Low friction coatings of diamond-like carbon with silicon prepared by plasma-assisted chemical vapor deposition, J. Mater. Res. 5 (2011) 2567-2571.

[67] S. Miyake, Tribological properties of hard carbon films: extremely low friction mechanism of amorphous hydrogenated carbon films and amorphous hydrogenated $\mathrm{SiC}$ films in vacuum, Surf. Coat. Technol. 54 (1992) 563-569.

[68] W.J. Wu, M.H. Hon, Thermal stability of diamond-like carbon films with added silicon, Surf. Coatings Technol. 111 (1999) 134-140.

[69] D. Hofmann, S. Kunkel, K. Bewilogua, R. Wittorf, From DLC to Si-DLC based layer systems with optimized properties for tribological applications, Surf. Coatings Technol. 215 (2013) 357-363.

[70] R. D'Agostino, Plasma Deposition, Treatment, and Etching of Polymers, Elsevier, San Diego, CA, 1990.

[71] M.T. Van Os, Surface modification by plasma polymerization: film deposition, tailoring of surface properties and biocompatibility, Film. (2000) 135.

[72] C. Casiraghi, F. Piazza, a. C. Ferrari, D. Grambole, J. Robertson, Bonding in hydrogenated diamond-like carbon by Raman spectroscopy, Diam. Relat. Mater. 14 (2005) 1098-1102.

[73] A.C. Ferrari, J. Robertson, Raman Spectroscopy of Amorphous , Nanostructured, Diamond-like Carbon, and Nanodiamond, Philos. Trans. Math. Phys. Eng. Sci. 362 (2004) 2477-2512.

[74] J.R. Shi, X. Shi, Z. Sun, S.P. Lau, B.K. Tay, H.S. Tan, Resonant Raman studies of tetrahedral amorphous carbon films, Diam. Relat. Mater. 10 (2001) 76-81.

[75] W.G. Cui, Q.B. Lai, L. Zhang, F.M. Wang, Quantitative measurements of sp3 content in DLC films with Raman spectroscopy, Surf. Coatings Technol. 205 (2010) 1995-1999.

[76] a. Ferrari, J. Robertson, Interpretation of Raman spectra of disordered and amorphous carbon, Phys. Rev. B. 61 (2000) 14095-14107.

[77] J. Fontaine, C. Donnet, a Erdemir, Tribology of Diamond-Like Carbon Films, 2008.

[78] P. Larkin, Infrared and raman spectroscopy: principles and spectral interpretation, n.d. 
[79] E. Mikmeková, Enhanced properties of carbon based coatings, 2014.

[80] M. Ban, T. Hasegawa, S. Fujii, J. Fujioka, Stress and structural properties of diamondlike carbon films deposited by electron beam excited plasma CVD, Diam. Relat. Mater. 12 (2003) 47-56.

[81] D.A. Shirley, High-resolution x-ray photoemission spectrum of the valence bands of gold, Phys. Rev. B. 5 (1972) 4709.

[82] D. Atwood, Soft X-rays and Extreame Ultraviolet Radiation: Principles and Applications, New York: Cambridge University Press, 1999.

[83] Y. Tang, Plasma and ion beam enhanced chemical vapour deposition of diamond and diamond-like carbon, PhD thesis Department of Mechanical Engineering, University of Saskatchewan, 2010.

[84] S. Ge, S. Wang, N. Gitis, M. Vinogradov, J. Xiao, Wear behavior and wear debris distribution of UHMWPE against Si3N4 ball in bi-directional sliding, Wear. 264 (2008) 571-578.

[85] H. Niakan, Synthesis and characterization of diamond-like carbon and dlc-mos2 composite thin films, $\mathrm{PhD}$ thesis in Department of Mechanical Engineering, University of Saskatchewan, 2014.

[86] S. Srinivasan, Y. Tang, Y.S. Li, Q. Yang, a. Hirose, Ion beam deposition of DLC and nitrogen doped DLC thin films for enhanced haemocompatibility on PTFE, Appl. Surf. Sci. 258 (2012) 8094-8099.

[87] W.C. Oliver, G.M. Pharr, An improved technique for determining hardness and elastic modulus using load and displacement sensing indentation experiments, J. Mater. Res. 7 (1992) 1564-1583.

[88] S. Lampman, Characterization and Failure Analysis of Plastics, ASM International, United State of America, 2003.

[89] K.L. Mittal, Polymer Surface Modification: Relevance to Adhesion, Volume 3, 2004.

[90] Z. Sanaee, S. Mohajerzadeh, K. Zand, F.S. Gard, H. Pajouhi, Minimizing permeability of PET substrates using Oxygen plasma treatment, Appl. Surf. Sci. 257 (2011) 2218-2225.

[91] A.V. Ã, M. Mozetic, A. Zalar, XPS study of oxygen plasma activated PET, Vacuum. 82 (2008) 248-251.

[92] M. Creatore, P. Favia, G. Tenuto, A. Valentini, NH 3 Plasma Treatments of PET for Enhancing Aluminum Adhesion, Plasmas Polym. 5 (2000) 201-218.

[93] K.N. Pandiyaraj, V. Selvarajan, R.R. Deshmukh, M. Bousmina, The effect of glow discharge plasma on the surface properties of Poly ( ethylene terephthalate ) ( PET ) film, Surf. Coat. Technol. 202 (2008) 4218-4226.

[94] K.N. Pandiyaraj, V. Selvarajan, R.R. Deshmukh, C. Gao, Adhesive properties of polypropylene ( PP ) and polyethylene terephthalate ( PET ) film surfaces treated by DC glow discharge plasma, Vacuum. 83 (2009) 332-339.

[95] L. Yang, J. Chen, Y. Guo, Z. Zhang, Surface modification of a biomedical polyethylene terephthalate ( PET ) by air plasma, Appl. Surf. Sci. 255 (2009) 4446-4451. 
[96] A. Bismarck, W. Brostow, R. Chiu, H.E.H. Lobland, K.K.C. Ho, Effects of Surface Plasma Treatment on Tribology of Thermoplastic Polymers, Polym. Eng. Sci. (2008) 1-6.

[97] H.T. Do, G. Thieme, R. Hippler, Energy distribution of ion species in $\mathrm{Ar} / \mathrm{CH} 4, \mathrm{Ar} / \mathrm{C} 2 \mathrm{H} 2$, and $\mathrm{Ar} / \mathrm{C} 3 \mathrm{H} 6$ radio-frequency plasmas, in: AIP Conf. Proc., 2005.

[98] X. Yan, T. Xu, G. Chen, S. Yang, H. Liu, Study of structure, tribological properties and growth mechanism of DLC and nitrogen-doped DLC films deposited by electrochemical technique, Appl. Surf. Sci. 236 (2004) 328-335.

[99] P. Slepi, N.S. Kasálková, E. Stránská, L. Ba, Surface characterization of plasma treated polymers for applications as biocompatible carriers, Polym. Lett. 7 (2013) 535-545.

[100] C. Casiraghi, a. C. Ferrari, J. Robertson, Raman spectroscopy of hydrogenated amorphous carbons, Phys. Rev. B - Condens. Matter Mater. Phys. 72 (2005) 1-14.

[101] K. Zhou, P. Ke, X. Li, Y. Zou, A. Wang, Microstructure and electrochemical properties of nitrogen-doped DLC films deposited by PECVD technique, Appl. Surf. Sci. 329 (2015) 281-286.

[102] T.W. Scharf, R.D. Ott, D. Yang, J. a. Barnard, Structural and tribological characterization of protective amorphous diamond-like carbon and amorphous $\mathrm{CN}$ [sub $\mathrm{x}]$ overcoats for next generation hard disks, J. Appl. Phys. 85 (1999) 3142.

[103] M. Lubwama, B. Corcoran, K. V. Rajani, C.S. Wong, J.B. Kirabira, A. Sebbit, et al., Raman analysis of DLC and Si-DLC films deposited on nitrile rubber, Surf. Coatings Technol. 232 (2013) 521-527.

[104] Z. Chen, J.N. Hay, M.J. Jenkins, FTIR spectroscopic analysis of poly(ethylene terephthalate) on crystallization, Eur. Polym. J. 48 (2012) 1586-1610.

[105] A. Laskarakis, C. Gravalidis, S. Logothetidis, FTIR and Vis-FUV real time spectroscopic ellipsometry studies of polymer surface modifications during ion beam bombardment, Nucl. Instruments Methods Phys. Res. Sect. B Beam Interact. with Mater. Atoms. 216 (2004) 131-136.

[106] Z. Seker, H. Ozdamar, M. Esen, R. Esen, H. Kavak, The effect of nitrogen incorporation in DLC films deposited by ECR Microwave Plasma CVD, Appl. Surf. Sci. 314 (2014) 4651.

[107] Y. Hayashi, K.M. Krishna, H. Ebisu, T. Soga, M. Umeno, T. Jimbo, Optical and structural properties of nitrogen doped amorphous carbon films grown by rf plasmaenhanced CVD, Diam. Relat. Mater. (2001) 1002-1006.

[108] J. Zhao, P. Lemoine, Z. Liu, A study of microstructure and nanomechanical properties of silicon incorporated DLC films deposited on silicon substrates, Diam. Relat. Mater. 10 (2001) 1070-1075.

[109] G.J. Wan, P. Yang, R.K.Y. Fu, Y.F. Mei, T. Qiu, S.C.H. Kwok, et al., Characteristics and surface energy of silicon-doped diamond-like carbon films fabricated by plasma immersion ion implantation and deposition, Diam. Relat. Mater. 15 (2006) 1276-1281.

[110] C.K. Park, S.M. Chang, H.S. Uhm, S.H. Seo, J.S. Park, XPS and XRR studies on microstructures and interfaces of DLC films deposited by FCVA method, Thin Solid Films. 420-421 (2002) 235-240. 
[111] R. McCann, S.S. Roy, P. Papakonstantinou, M.F. Bain, H.S. Gamble, J. a. McLaughlin, Chemical bonding modifications of tetrahedral amorphous carbon and nitrogenated tetrahedral amorphous carbon films induced by rapid thermal annealing, Thin Solid Films. 482 (2005) 34-40.

[112] C. Donnet, A. Erdemir, Tribology of Diamond-Like Carbon Films, Springer US, 2008.

[113] K. Kadiyala, Characterization and Tribological Behavior of Diamond-Like Carbon and Nitrogen-Doped Diamond-Like Carbon Thin Films, MSc thesis in Department of Mechanical Engineering, Nagarjuna University, (2006).

[114] J. Wang, J. Pu, G. Zhang, L. Wang, Tailoring the structure and property of silicon-doped diamond-like carbon films by controlling the silicon content, Surf. Coatings Technol. 235 (2013) 326-332. 